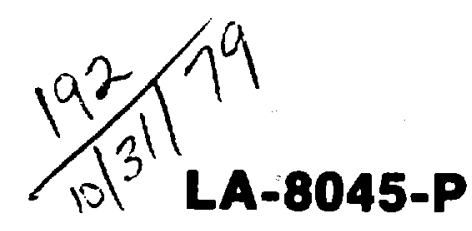

Proposal

\title{
Proposal for FRX-C and Multiple-Celf Compact Torus Experiments
}

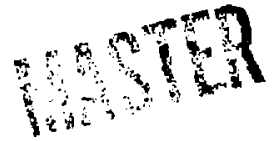

to

$\frac{2}{5}$ 


\title{
Proposal for FRX-C and Multiple-Cell Compact Torus Experiments
}

submitted to

\author{
Office of Fusion Energy \\ Department of Energy \\ Washington, DC 20545
}

by

H. Dreicer

for

Staff of the Controlled Thermonuclear Division Los Alamos Scientific Laboratory Los Alamos, New Mexico 87545 
PREFACE $\quad$ vi

ABSTRACT 1

EXECUTTVE SUMMARY 2

(3.110) SCIENTIFIC SCOPE

Incroduction . . . . . . . . . . . . . . . 5

Overview of CT Research................... 6

LASL CT Program. . . . . . . . . ........ 3

Status of Field -Reversed Theta-Pinch Physlcs . . . . . 10

The FRX-C Scaling Experiment . . . . . . . . . . 15

The Scylla IV-P Multiple-Cell Experiment . . . . . . . . 19

Future Plans. . . . . . . . . . . . . . . 22

(3.120) ENGINEFRTNG DESCRIPTION

The FRX-C Experiment . . . . . . . ....... 24

The Scylla IV-P Multiple-Cell Expertment . . . . . . . 27

Plasna Diagnostics................ 32

(3.130) TOTAL ESTLMATED COST

$\begin{array}{ll}\text { (3.140) MILESTONES } & 37\end{array}$

(3.150) MANPOWER REQUIREMENTS 38

References. . . . . . . . . . . . . . 39

Appendix A--LASL Compact Torus Progam Plan . . . . . . . 41

Appendix B--Review of Previous Experiments . . . . . . 69

Appendix C--Status of Field-Reversed Theta-Pinch Theory. . . 85

Appendix D-Field-Reversed Theta-Pinch Scaling . . . . . 92

Appendix E--Recent FRX-A and FRX-B Results . . . . . . 103 
PREFACE

Compilation of this document is primarily the effort of R. E. Siemon with help from H. Dreicer, R. K. Linford, and A. R. Sherwood. Particular credit goes to D. C. Barnes and C. E. Seyler for help in clarifying various aspects of the theory, to R. F. Gribble for designing the FRX-C electrical circuit, to K. W. Hanks for mechanical englneering advice, to K. F. Mckenna for planning the Scylla IV-P modifications, to T. R. Jarboe for planning diagnostics, and to W. E. Quinn for much advice including estimates of cost and schedule. Other contributors to experimental aspects include W. T. Armstrong, R. R. Bartsch, R. J. Commisso, and C. A. Ekdahl. Useful discussions are also acknowledged with D. A. Baker, J. N. DiMarco, R. A. Gerwin, R. L. Hagenson, G. Miller, K. S. Thomas, and others of the CTR-Division staff. Much gratitude is owed N1kk1 Goldman for her work on preparation of this manuscript; particularly those weekend hours spent on the numerous last-minute revisions. 
PROPOSAL FOR FRX-C AND MULTIPLE-CELL COMPACT TORUS EXPERIMENTS

by

LASL CTR-Division Staff

ABSTRACT

A Compact Torus (CT) is a configuration for plasma confinement that offers possible engineering advantages for fusion power generation such as small size, simple blanket geometry, natural divertor, and spatially separable functions of plasma production and fusion energy generation. Two experiments to study the physics and technology of some particular CT configurations are proposed here as part of the LASL Compact Torus Program Plan. One experiment, FRX-C, is designed to study CT stability and transport properties by scaling the parameters of the existing FRX-B field-reversed theta-pinch experiment to higher temperatures, larger size, and increased plasma lifetime. The second experiment, a modification of the existing Scylla IV-P device, would form a linear array of CTs with the aim of understanding the effect on CT transport of improved plasma confinement on the open field lines outside the separatrix, as well as other multiple-cell effects. 


\section{Definition of Compact Torus (CT)}

A toroidal plasma configuration in which internal plasma currents help to create the confining magnetic field eliminating the need for a toroidal vacuum vessel or toroidal field coils.

\section{Major Goal}

To understand the physical properties of CT configurations in order to develop a viable reactor embodiment.

\section{Potential Reactor Advantages}

Small size and untt power

Simple blanket geometry

Efficient use of magnetic field; includes natural divertor

spatially separable plasma production and fusion energy generation

\section{CT Issues to be Resolved}

Optimum CT configuration -- Aspect ratio, $a / \rho_{1}$, toroldal field, etc. Production method -- Particle beams or pulsed plasma techniques Stability requirements -- Geometry, proximity of metal walls, FLR, etc. Transport -- Pressure profile effects, microturbulence, etc. Heating - Neutral beams, magnetosonic, adiabatic compression, etc. Reactor embodinent(s) -- Depends on resolution of above issues

\section{LASL'S Research Addressed to Key CT Issues}

Single cell studies (production, stability, transport)

FRX-B -- Successful field-reversed experiment

CTX trapping facility (under construction)

Sources -- Magnetized plasma gun (with toroidal B)

-- Proposed FRX-C (without toroidal B)

Yultiple cell studies (transport -- pressure profile effects)

Proposed modification of Scylla IV-P 
Objectives of Proposed Experiments

FRX-C - - Scaling from FRY-B to higher temperature and larger $a / \rho_{1}$

-- Increased lifetime

-- Wider range of flasma parameters

Multiple cell experiment

-- Improved confinement with increased pressure on separatrix

-- Multiple mirror confinement on open field lines

-- Study variable number of cells

-- Direct comparison with single cell results (FRX-B).

\section{LASI Prograin}

Builds on the present LASL FRX-A and FRX-B results

Continues theoretical studies of CT plasma properties

Shares physics 1ssues with RFP Program

Includes CT reactor studies

Utilizes LASL expertise and facilities

Interactions with other Programs

Compact Torus (Krasnaya--Pachra)

Spheromak (Princeton, U. Maryland)

Field Reversed Mirror (LLL)

CT production by particle beams (Cornell, NRL, UC Irvine)

Linus (NRL)

\section{Costs (K\$)}

FRX $-\mathrm{C}$ :

\begin{tabular}{|c|c|c|c|c|}
\hline FRX - C : & FY80 & FY8 1 & FY82 & To \\
\hline Manpower & 1245 & 1410 & 1840 & \\
\hline Tota1 & 1835 & 1710 & 2190 & \\
\hline Multiple cell: & 1st FY & & & Total \\
\hline Manpower & 600 & & & 1440 \\
\hline Tota1 & 960 & & & 1930 \\
\hline
\end{tabular}


Schedule

FRX-C: Begin operating October, 1980 (assumes Sept. 1979 approva1)

Multiple cell: Begin operating September of first funded flscal year 


\section{(3.110) SCIENTIFIC SCOPE*}

\section{Introduction}

The purpose of this document is to propose and describe two experimental projects that are important scientific and technical steps in the LASL Compact Torus (CT) Program Plan. These are

1) The FRX-C Scaling Experiment, and

2) The Scylla IV-P Field-Reversed Multiple-Cell Experiment.

A Compact Torus is a toroidal plasma configuration (see Fig. 1) in which effective use is made of internal plasma currents, rather than currents in external conductors, to help create the confining magnetic fields. Replacement of external with internal currents in a CT does away with the need for a toroidal vacuum vessel and the usual toroidal magnetic field coils linking the plasma. It is anticipated that this modification could pave the way for a greatly improved fusion reactor design. In such a design the reactor's blanket might be cylindrical. or spherical rather than toroidal, and this would simplify the construction and maintenance. The system would possess natural divertor action because the CT has a topology of open and closed magnetic fleld lines separated by a separatrix surface. While the need for auxiliary heating cannot be ruled out, the possibility of ohmically heating a CT to ignition exists. Some equilibria may allow the use of deuterium-based advanced fuels since the beta may be sufficient to avoid unacceptably high levels of synchrotron radiation. Finally, the elimination of magnetic field coils linked with the $C T$ plasina provides the freedom to translate $\mathrm{CT}^{\prime} \mathrm{s}$ in space, and makes possible the spatial separation between the CT formation region and the region where the fusion burn occurs. These and other CT reactor design features have not yet been subjected to a rigorous system study. However, the outlook for a superior advanced fusion reactor design is sufficiently promising to warrant a strong CT research and development effort.

\footnotetext{
*Numbers 3.110 to 3.150 are requested in the Procedures Governing the Initiation and Fabrication of Major Projects within the Controlled Thermonuclear Research Program (ERDA-5, Feb. 1975).
} 


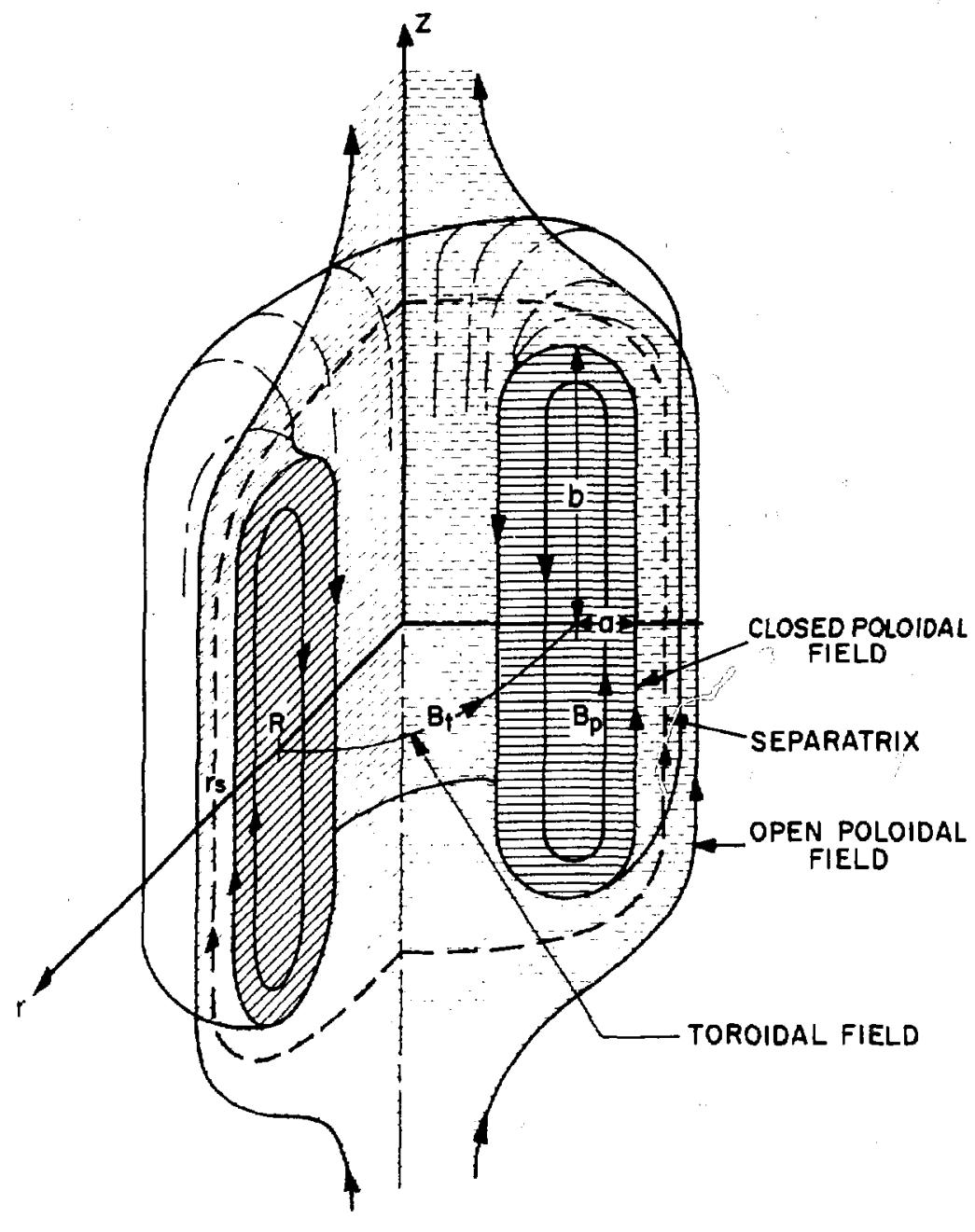

Fig. 1.

Compact torus schematic showing the geometrical parameters $\mathrm{R}, \mathrm{a}$, and $\mathrm{b}$, and the fields $B_{p}$ and $B_{t}$. For some $C^{\prime}{ }^{\circ} s, B_{t}=0$.

\section{$\because$ rriew of CT Research}

The national CTR research effort contains a diverse set of CT programs. Fiese include the Astron concept of Christofilos'1 now being studied at Corre $1 I^{2}$ and NRL, ${ }^{3}$ Fleld-Reversed Mirror formation by neutral beam injection at LLi, 4 conical theta pinches, ${ }^{4}, 6,7$ field-reversed theta pinches without axial currents studied at LASL in the FRX-A and FRX-B experiments ${ }^{8}$ and elsewhere (see 
Appendix B), field-reversed theta pinches with axial current being studied at University of Maryland, ${ }^{9}$ magnetized Marshall guns orginally studied $\Rightarrow$ by Alfvén, 10 and now being pursued by LLL and LASL (see Appendix A), rotating e-beam formation at NRL ${ }^{11}$ and UC Irvine, 12 and the Spheromak concept under study at Princeton, 13 etc. The LINUS ${ }^{14}$ approach at NRL a iso includes the isse of a CT configuration for plasma confinement. Theoretical and experimental results from these programs are beginning to form a more unified understanding of the CT configuration. This broad understanding is essential before wise choices can be made as to which type(s) of CT would provide the basis for the most attractive reactor $(s)$.

CT research will involve substantial scientific and technological interaction with the major MFE fusion programs. Theoretical similarities with other axisymmetric systems such as Tokamaks and RFP's should "result in mutually beneficial cooperation. In addition, the plasma behavior on the open field lines should be strongly related to mirror physics. Heating techniques, magnetic field systems, and other technologies are likely to be similar to those used in other programs, depending on the particular CT configuration."

As indicated by the above examples, the CT concept encompasses a broad class of configurations. Two important parameters that can be used to characterize a CT and its possible states of equilibrium are $a / \rho_{i}$ (where a is the minor radius of the plasma and $\rho_{1}$ is the jon Larmor radius in the region of the maximum $B-f i e l d$ ) and $B_{t m} / B_{p m}$ (where $\hat{B}_{t m}$ and $B_{p m}$ are the maximum toroidal and poloidal fields). The value of $a / \rho_{i}$, can range between $a / \rho_{i}<1$ as $i$ a thin Astron layer, to $a / \rho_{i} \gg 1$ as in a Spheromak. The value of $B_{t m} / B_{p m}$ can range from zero as in the Field-Reversed Theta-Pinch Experiments (no internal toroidal field) to $\mathrm{B}_{\mathrm{tm}} / \mathrm{B}_{\mathrm{pm}}>=-1$ as in the spheromak concept. "A multifaceted, national CT program would achieve a unified understanding of this broad class of configurations and their "equjibria. Understanding the stability and transport properties of CT's will allow the selection of the most attractive reactor embodiment(s) (see Appendix A) as well as selection of specific production and heating methods. 


\section{LASL CT Program}

The main objective of the LASL CT Progam Plan is to produce several CT configurations and to study their physical properties in a series of conrdinated steps so that the possibility of viable reactor embodiments can be definitely evaluated. In carrying out this task LASL would pursue those areas of CT physics research that are effectively complimented by the CT research efforts at other laboratories. The LASL CT Progam Plan (see Appendix A) thus contributes to the above described need for a unified understanding of CT configurations. This section of the proposal will describe only the broad outline and main features of that plan. The reader is referred to the Appendix $\therefore u r$ a more detailed description of the physics and programmatic considerations that entered into its development.

Although there may be several ways to produce $\mathrm{CT}^{\prime} \mathrm{s}$ both with and without F, it seems reasonable that LASL employ existing facilities and technologies (1) obtain physics results on the shortest possible time scale. For example, since L.ASL does not presently possess the relevant beam technology for the sitron approach to $\mathrm{CT}^{\circ} \mathrm{s}\left(\mathrm{a} / \rho_{1}<1\right)$, and since the concept is being studied Cisiwliere, the LASL CT studies will be focused on equilibria with $a / \rho_{i}>1$. Pinis range of $a / \rho_{i}$ can be studied with the LASL FRX-A, FRX-B, and proposed $\because K-X^{\prime}$ systems which produce $C T$ 's with $B_{t m} / B_{p m}=0$. The case with $B_{t m} / B_{p m} \sim 1$ arid $a / k_{1}>1$ is also being examined at LASL with the magnetized Marshall gun which is now under construction.

The use of these particular sources allows LASI to begin immediately on CT research. Nltimately, when the physics of the CT configuration is better understood, it may be unnecessary to employ the high-voltage pulsed-power techniques which are presently being used. Alternatively, it may be acceptable co use high voltage in a reactor embodiment because a CT toroid can be formed in one spatial region and then be translated and trapped in a separate region. Thus the high voltage is removed from the vicinity of the first wall surrounding the fusion plasma much as it is with neutral beam technology.

The central facility of the LASL CT experimental program for single cell configurations will be the CTX now under construction. This facility is designed to provide the magnetic field and vacuum conditions required to sustain CT's for longer times than are possible in present LASL experiments. It includes a $1.5-\mathrm{m}$ diameter, $4.5-\mathrm{m}$ long vacuum tank with a $10-\mathrm{kG}$ magnetic 
field for trapping and confining CT plasmoids injected from either end. It is planned that CT's from the magnetized Marshall gun be injected from one end and that tori from the proposed FRX-C experiment be injected from the other end. The parallel development of more than one type of injection source, as recommended by the Ad-Hoc Review Committee for the magnetized Marshall gun, will make possible a comparison of CT configurations with and without coroidal magnetic field and in general improves the likelihood of developing a long-lived CT configuration. A substantial diagnostic system is being assembled for the detailed study of the stability and transport properties of CT's produced by both sources.

In parallel with the studies of single cell CT configurations in the CTX facility, it is proposed to study the multiple-cell configuration in a modified version of the existing Scylla IV-P experiment.

Auxiliary heating studies are also planned. Several heating techniques appear attractive with the choice dependent on the specific reactor embodiment. Heating studies that can be made on existing experiments are restricted to those capable of high powers because of the short life times $(<100 \mu s)$ that presently exist. Two attractive possibilities are adiabatic compression and magnetosonic heating. When plasma lifetimes of $\gtrsim 1 \mathrm{~ms}$ are achieved, neutral beam heating will become attractive. Although preliminary heating experiments might be carried out in the existing small factlities, the major heating studies are planned for the CTX system now under construction.

The expectation of producing longer-lived CT's is based not only on the experimental results from FRX-A and FRX-B, but also on the very substantial theoretical advances during the past year. The observed gross MHD stability of $\mathrm{CT}^{\prime}$ 's without an internal toroidal magnetic field $\mathrm{B}_{t}$ is now understood, as well as the source of plasma rotation and the resulting $n=2$ rotational instability (see Appendix $\mathrm{C}$ ), where $\mathrm{n}$ is the toroidal mode number. Continued theoretical progress is expected to provide a more detailed understanding of the stability and transport associated with specific CT equilibria and a unifled understanding of various CT experiments.

Reactor studies are important to guide the CT program and a number of reactor embodiments have already been proposed. ${ }^{14}, 15,16,17$ This year the LASL Magnetic Fusion Reactor Systems Studies effort is undertaking a low-level (Level III) study of generic CT reactor operating scenarios, technology requirements, constraints, and energy balances in order to establish the 
groundwork for more detalled and focused reactor conceptual designs. As more is learned experimentally and theoretically about CT plasma physics, more realistic evaluations and more credible reactor extrapolations can be made. In turn, the identification of attractive CT reactor embodiments will guide the progression of experimental research. Evaluation at LASL of CT reactors on a common basis with other alternative concepts makes possible a useful comparison of relative advantages and disadvantages.

The LASL CT program recognizes that the FRX-A and FRX-B experiments demonstrate a promising method of CT production. The proposed experiments will extend these results and will answer many of the cructal questions on the stability and transport properties of CT's with $B_{t m} / B_{p m}=0$. In particular, the proposed FRX-C experiment wIIl study $C T$ stability and transport properties by scaling the parameters of the existing FRX-B experiment to higher temperature, larger size, and increased plasma lifetime. In addition, the Multiple-Cell Compact Torus experiment will study plasma transport along open field lines outside the separatrix and the resulting improvement in CT plasma confinement. The improvement of open-field-ine confinement is a result of multiple mirror effects which will be obtained in the proposed modification of the existing Scylla IV-P experiment.

The proposed experiments are modest in size and make use of existing facilities, avallable components, and established technology to as great an extent as possible. They form an important part of a unified LASL effort to expand the CT physles data base so that the potential of CT's to our national fusion program can be evaluated.

\section{Status of Field-Reversed Theta-Pinch Physics}

This section contalus a general summary of fleld-reversed theta-pinch physics followed by a more specific discussion of results from the present LASL FRX programs. A review of the extensive literature on this type of experiment is given in Appendix $B$. It shows clearly that $C T$ configurations have been generated by theta-pinch techniques in many laboratories with plasma lifetimes ranging from 1-100 $\mathrm{Hsec}$. A careful examination of the results reveals that the lifetime in recent experiments is much longer than expected from estimates of 
MHD growth rates based on Alfvén speed and characteristic size or estimates based on open-field-1ine confinement.

Theoretical Interpretation of field-reversed theta-pinch experiments, summarized in Appendix $\mathrm{C}$, has been improved in recent years. The observed gross stability to whole-body MHD displacements is now understood in terms of geometrical effects and the proximity of metal walls. The rotational $n=2$ mode, often observed to occur in experiments near the termination of plasma confinement, is presently thought to be a consequence of non-classical plasma transport. The rotation arises only after a significant part of the plasma has escaped, implying that the basic limitation on plasma confinement is particle loss and is not due to the rotation itself.

The following main points can be summarized from the experimental and theoretical work to date:

- A CT without toroidal field can be created with theta-pinch techniques. The resistive tearing mode can be controlled by proper vacuum procedures, preionization methods, and experimental design.

- A weakly comprensed CT inside a metal wall is stable to whole-body MHD displacements.

- The CT displays a quiescent phase usually terminated by a growing $\mathrm{n}=2$ mode most likely caused by rotation resulting from non-classical transport.

- The confinement of plasma and energy during the quiescent phase is superior to plasma confinement in an open field line configuration. Further theoretical and experimental work will clarify the observed losses and determine the confinement scaling.

- Translation of the CT from one coll region to another has been successfully demonstrated.

These favorable results provide a major impetus for the experiments proposed here. 
Two LASL experiments, $F R X-A$ and $F R X-B$, have been successful in clarifying many features of field-reversed theta-pinch operation. ${ }^{8}, 18,19$ (Reprints of these references are attached in Appendix E.) The proposed experiments are based on the FRX results and on new theoretical understanding of FRX behavior. Figure 2 shows the arrangement of a field-reversed configuration in a theta-pinch coil. Following the application of a reversed bias field and preionization, the formative phase, depicted in Fig. 3, starts with a radial implosion of the plasma driven by the fast rising external field. Subsequently in the first microsecond a tearing mode at the ends of the coil allows magnetic field lines to reconnect forming an elongated plasma torus. Within a few microseconds the plasma contracts axially to form the equilibrium shown in

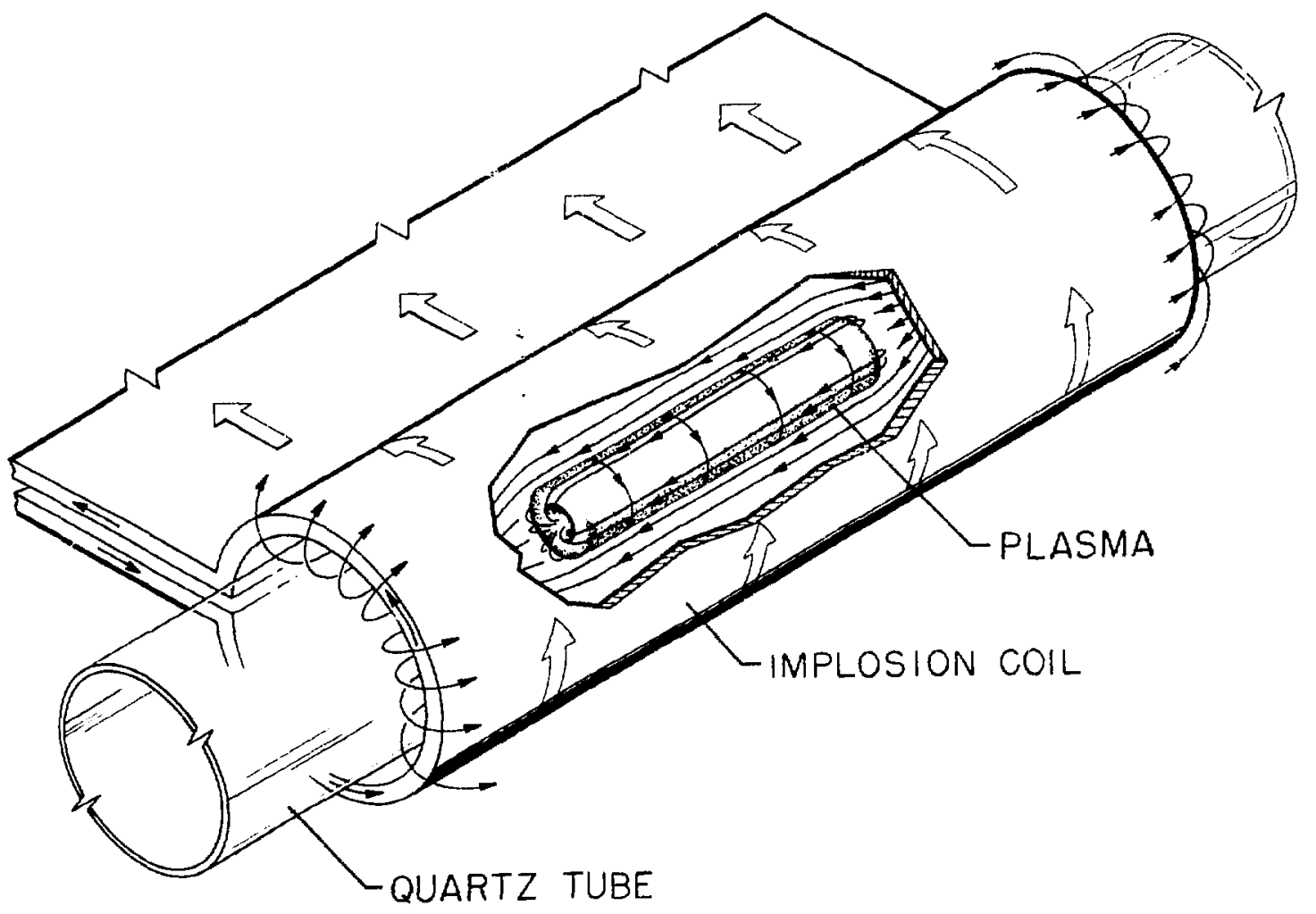

Fig. 2 .

Arrangement of theta-pinch coil, discharge tube, and CT plasma configuration obtained wth reversed blas field. 

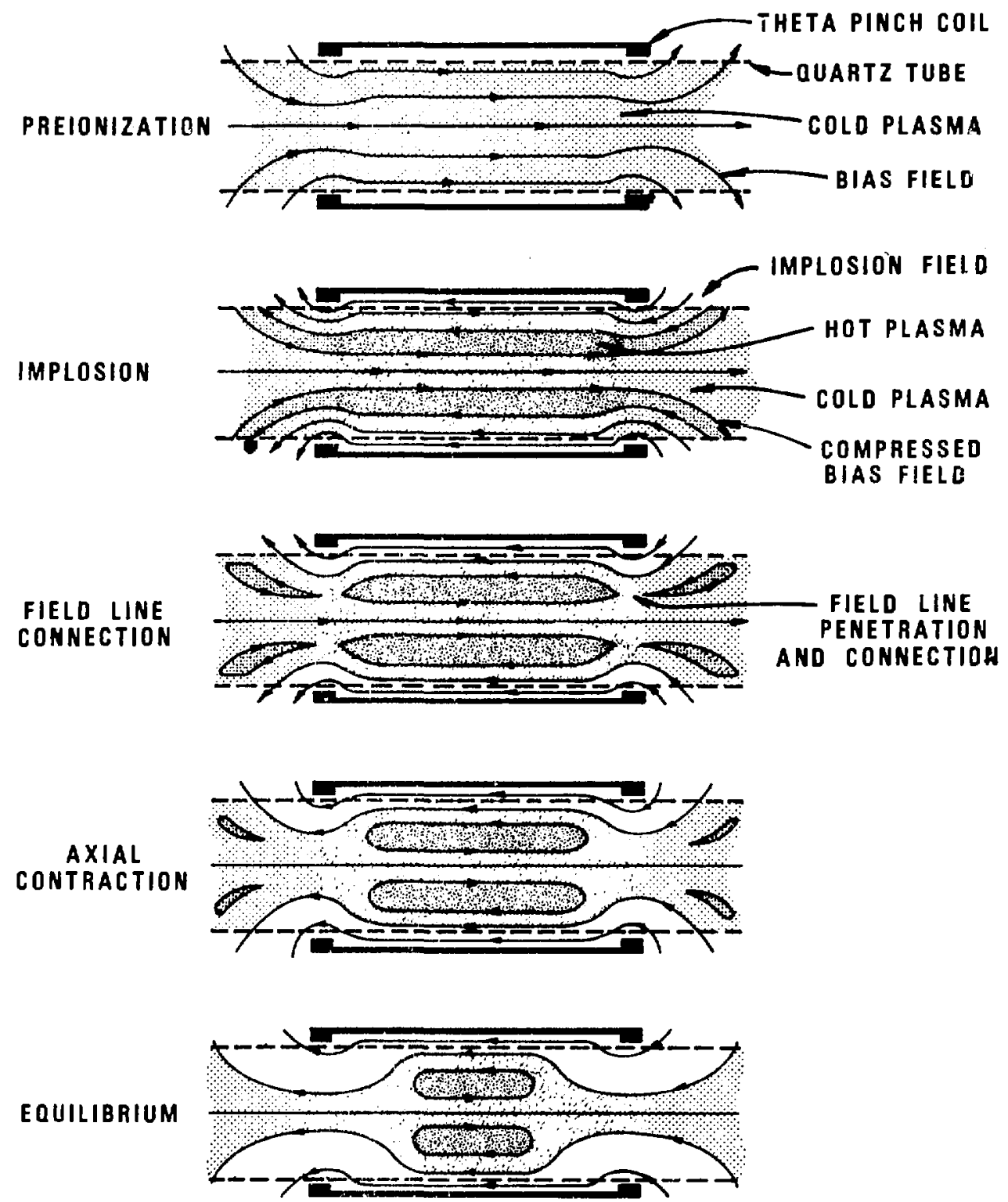

Fig. 3 .

Formation sequence of a $\mathrm{CT}$ in a field-reversed theta pinch.

Fig. 2. A separatrix defines the plasma boundary inside of which field 1ines are closed and outside of which the magnetic lines are open extending out to the discharge tube walls forming a natural divertor for the CT configuration. 
Eigure 4 shows typical magnetic field behavior outside the discharge tube in the midplane of the coll obtained with a probe. Prior to the implosion, which occurs at $t=0$ on $\mathrm{Fig}$. 4, a rapid oscillation of the reversed bias field
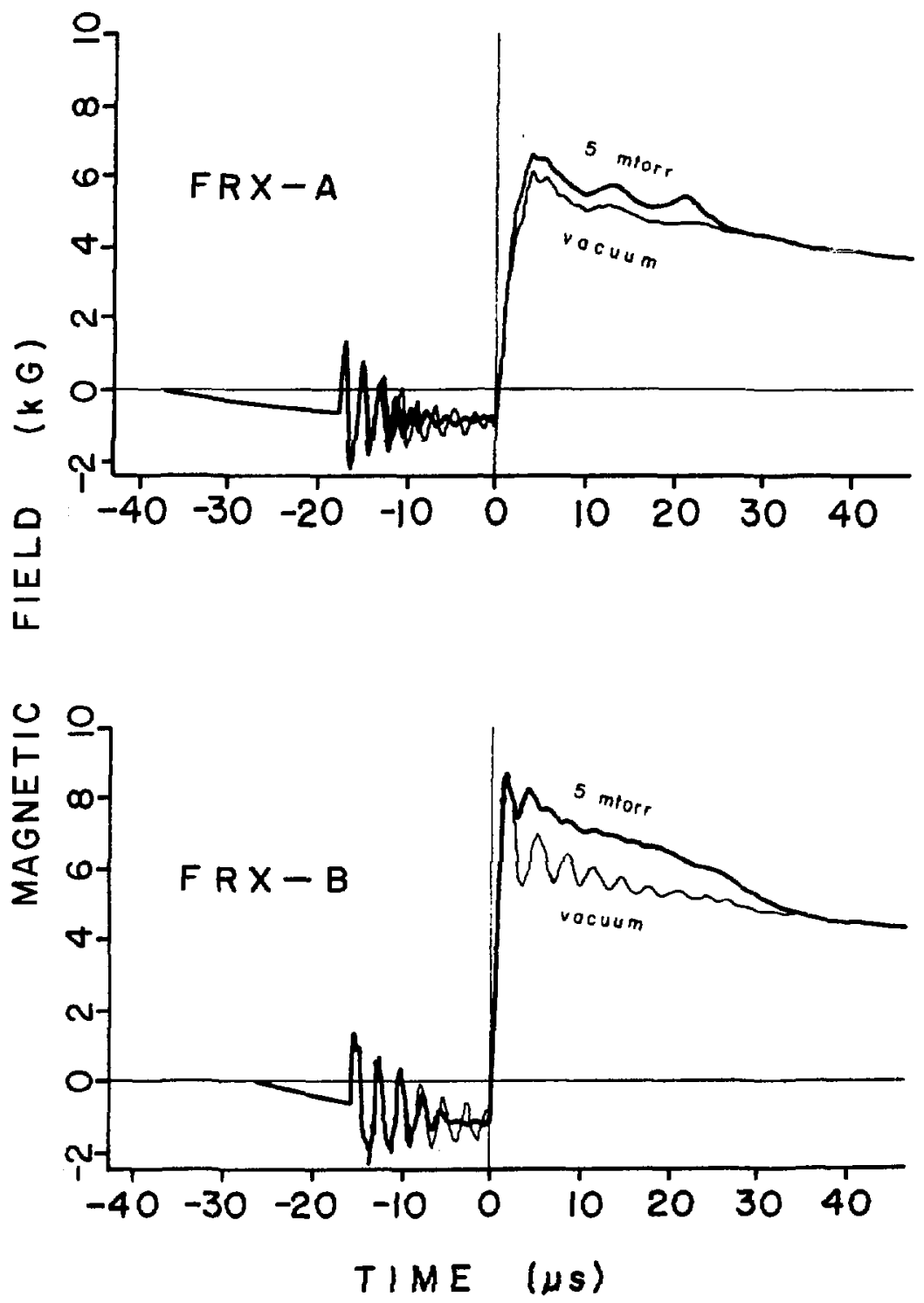

Eig. 4.

Magnetic field time history obtained with a probe outside the discharge tube at the coll midplane. 
preionizes the filling gas. After the implosion and axial contraction, a quiescent phase is observed which persists for tens of microseconds. The existence of a CT equilibrium is spen as an increase in the value of local magnetic field strength, when plasma is present, over the vacuum value as shown in Fig. 4. The plasma lifetime is defined as the duration of this increased field strength determined with a magnetic probe. The very long lifetimes observed in these experiments (20-30 $\mu$ s in Fig. 4) are evidence that a CT has been formed since simple plasma streaming out the ends would limit the lifetime to about $3 \mu \mathrm{s}$. The CT plasma is clearly MHD stable during the quiescent phase until, in most cases, an $n=2$ mode appears, as depicited on image converter photographs somewhat before the end of the configuration lifetime.

Some tentative but encouraging conclusions regarding lifetime can be drawn from the FRX-A and FRX-B experiments. Comparisons of FRX-A and FRX-B results show that for a given $a / \rho_{i}$, the lifetime depends weakly upon temperature. In FRX-B the lifetime is found to increase monotonically with increased $a / \rho_{i}$ (as the temperature is lowered by increasing the fllling pressure). These are encouraging results as they indicate that confinement will be improved in a larger size device. A loss of stability resulting from reduced finite-Larmor-radius effects is so far not indicated. The subject of transport is the least thoroughly studied area both experimentally and theoretically, but recent calculations by Hamasaki ${ }^{20}$ including anomalous transport resulting from the lower hybrid drift instability are in general agreement with the FRX-A and FRX-B experimental results.

Experimental effort must now be directed towards increasing the plasma lifetime beyond the limits so far observed. A better understanding of these limits will come with continuing theoretical effort and more complete diagnostics being implemented on FRX-B. The plans to improve the confinement time with larger experimental devices are described in the next two sections.

\section{The FRX-C Scaling Experiment}

The purpose of the FRX-C experiment is to increase the plasma lifetime and to establish the scaling of confinement time with size and plasma temperature. The FRX-C device, shown in Fig. 5, will be approximately twice the size of FRX-B and utilizes higher voltage. There are three main reasons for these 


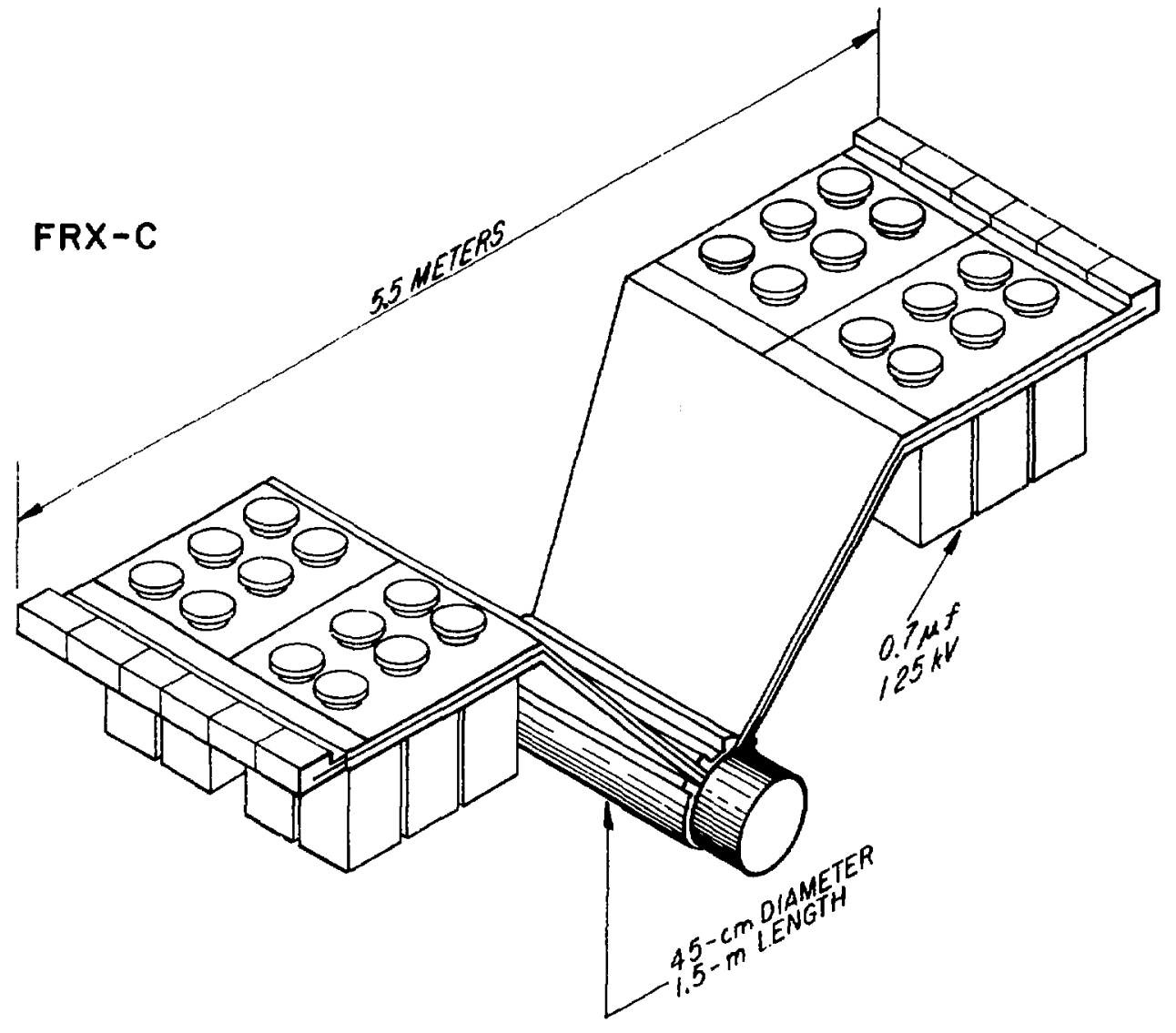

Fig. 5 .

Arrangement of FRX-C double-fed coll, collector plates, and main bank capacitors.

design choices: 1) The increased voltage gives higher temperatures; 2) The ratio $a / \rho_{1}$ will be increased by approximately a factor of two; 3) The accessible range of filling pressure, which determines the range of temperature and $a / \rho_{i}$ in a given experiment, will be increased. These changes are expected to result in a reduction of cross-fleld losses by an increase of $a / o_{1}$ as found in the FRX-B experiments.

The detailed scaling laws used in selecting the experimental parameters for FRX $-C$ are derived in Appendix D. An examination of the literature summarized in Appendix B indicates that the longest confinement times are obtained with minimum adiabatic compression following the Implosion phase (see Fig. D-2). The explanation is uncertain, but it is possibly a consequence of 
the way the CT equilibrium shape changes as the plasma is compressed. When the magnetic field increases in a coll of fixed dimension, the plasma density gradient near the separatrix increases. 21 Consequently, plasma transport is enhanced and the lifetime is shortened. Therefore, FRX-C is designed to have a sma11 magnetic compression, approximately the same as in FRX-B, which necessarily places the burden of heating on the implosion phase and implies a rapidly rising magnetic field and a high voltage on the driving coil. Using previously established scaling laws for theta-pinch heating, it is possible to estimate the parameters expected in $F R X-C$ and the variation with filling pressure. These estimates are presented in Table $I$ based on the equations in Appendix $D$ and experimental values $F$ rom FRX-B. Note that the only experimental data are those for FRX-B in the left most column corresponding to 9-mtorr initial pressure. The other columns give expected parameters calculated according to the scaling laws given the magnetic field, dimensions, and filling pressure at the top of each column. The estimates of temperature may be uncertain by as much as a factor of two since processes such as radiation losses, variations in magnetic field waveform resulting from plasma-circuit interactions, and heating by axtal compression have been oversimplified or left out.

The parameter $a / p_{i}$ is important both for its role in cross-field transport as well as its role in finite-Larmor-radius (FLR) stabilization. In a particular device this parameter is most easily varied by changes in filling pressure as indicated in Table $I$. In FRX-B the upper end of the pressure range is limited to about 17 mtorr, since at the higher pressures the temperature drops, the plasma becomes collisional ( $\omega \tau<1$ ), and the CT configuration rapidly diffuses away. The second column of Table I corresponding to a pressure of 90 mtorr shows the parameters predicted by the scaling laws given in Appendix D. No experimental data are avallable for the higher pressure. The essential point is that a large variation of filling pressure appears achievable with a collisionless plasma in FRX-C, whereas it does not appear achievable in FRX-B in agreement with the limits found experimentally.

Some characteristic times are included at the bottom of Table $I$. The expression for confinement time $\tau_{\perp}$ represents an approximate upper 1 imit on the energy confinement time resulting from cross-field ion thermal conduction. 15 The limitations on confinement in CT equilibria without $B_{t}$ are probably related to non-classical transport mechanisms such as the lower hybrid drift mode or 
TABLE I.

Comparison of FRX-B and FRX-C Parameters

\begin{tabular}{|c|c|c|c|c|}
\hline Source voltage $(\mathrm{kV})$ & $\frac{\text { FRX-B }}{45}$ & & $\frac{\text { FRX }-C}{250}$ & \\
\hline Final B-field ( $k G)$ & 7.5 & & 9 & \\
\hline Risetime ( $\mu s)$ & 1.8 & & 1.8 & \\
\hline Decay time ( $\mu s)$ & 150 & & 200 & \\
\hline Discharge tube diameter & $(\mathrm{cm})$ & & 40 & \\
\hline Coll length (cm) & 100 & & 150 & \\
\hline Fill pressure (mtorr) & 9 & 90 & 2.4 & 24 \\
\hline Line density, $N_{0}\left(\mathrm{~cm}^{-1}\right)$ & $1.9 \times 10^{17}$ & $1.9 \times 10^{18}$ & $2 \times 10^{17}$ & $2 \times 10^{18}$ \\
\hline Bias field (kG) & 1.8 & 3.2 & 1.6 & 2.8 \\
\hline $\mathrm{K}_{1}^{*}$ & $4 \cdot 7$ & $2 \cdot 3$ & 5.6 & 3.2 \\
\hline Major radius, $R(\mathrm{~cm})$ & $4 \cdot 0$ & 5.7 & $6 \cdot 7$ & 8.9 \\
\hline Minor radius, a $(\mathrm{cm})$ & 1.7 & 1.7 & 3.4 & 3.4 \\
\hline Length, $2 b(\mathrm{~cm})$ & 36 & 36 & 54 & 54 \\
\hline Electron temp. (ev) & 100 & 16 & 480 & 85 \\
\hline Ion temp. (ev) & $240 \mathrm{eV}$ & $38 \mathrm{eV}$ & $1140 \mathrm{eV}$ & $200 \mathrm{eV}$ \\
\hline Final density, $n_{f}\left(\mathrm{~cm}^{-3}\right)$ & $4.1 \times 10^{15}$ & $2.6 \times 10^{16}$ & $1.2 \times 10^{15}$ & $6.8 \times 10^{15}$ \\
\hline$a / p_{i}$ & 4 & 10 & 5 & 11 \\
\hline$\omega_{1} \tau_{1}$ & 71 & 0.7 & $3 \times 10^{3}$ & 40 \\
\hline$\tau_{\text {obs }}$ & $35 \mu \mathrm{s}$ & $?$ & $?$ & $?$ \\
\hline$\tau_{\mathrm{MHD}}=\frac{(a b)^{1 / 2}}{V_{A}}$ & $0.4 \mu \mathrm{s}$ & $0.9 \mu \mathrm{s}$ & $0.3 \mu \mathrm{s}$ & $0.7 \mu \mathrm{s}$ \\
\hline$\tau_{\text {Bohm }}=a^{2} / D_{B}$ & $15 \mu s$ & $92 \mu \mathrm{s}$ & $15 \mu \mathrm{s}$ & $82 \mathrm{~ms}$ \\
\hline$\tau_{\perp}=\left(a / \rho_{i}\right)^{2} \tau_{i i}$ & $32 \mu \mathrm{s}$ & $2 \mu s$ & $1300 \mu s$ & $100 \mu \mathrm{s}$ \\
\hline
\end{tabular}

* Magnetic compression equal to final field divided by maximum blas fleld (see Appendix D).

MHD turbulence of a short wavelength nature, and consequently, the performance of FRX $-C$ is not easily predicted. The experiment is important in establishing 
the scaling laws of confinement time with size and temperature, a needed step towards understanding the physics of CT's without toroidal field. The new parameter regime expected for the $\mathrm{FRX}-\mathrm{C}$ device has not been available in other CT experiments and will permit studies of plasma transport and stability in a collisionless fusion-relevant regime.

\section{The Scylla IV-P Multiple-Cell Experiment}

The objective of a multiple-cell experiment is to improve the confinement of plasma which would otherwise leave along open field lines that surround the CT plasma outside of the separatrix. Improved plasma confinement in this region is possible because of multiple magnetic mirror effects. The consequence of the improved confinement is an increase of plasma pressure on and near the separatrix, thereby reducing the sharp pressure gradients which would otherwise tend to form at the plasma boundary. Reducing the pressure gradient may prevent short wavelength interchange modes (see Appendix $C$ ) or may alter favorably the plasma transport processes. Thus an improvement of CT plasma confinement within the closed field region is expected to result from the modifled conditions at the separatrix.

Various reactor embodiments have been proposed which make use of multiple CT cells either for an improvement in energy balance 16 or the expected improvement in plasma confinement. 17 By modifying the existing 5-m Scylla IV-P theta pinch to have parameters essentially identical to the present FRX-B experiment, except for the factor of five in coil length, it is possible to compare in a meaningful way the data base for single cell operation with the multiple-cell results.

Particles on open field lines have been essentially unconfined in field-reversed experiments carried out to date. The magnetic field in many cases is slightly mirrored $(M \sim 1.2)$ but not enough to significantly change the estimated free-streaming time of lons, i.e.,

$$
\tau_{F S}=\frac{M_{1 / 2}}{v_{i}} \text {. }
$$


Here $M$ is the magnetic mirror ratio, $L_{1 / 2}$ is the coll half-length, and $v_{i}$ is the ion thermal speed. For FRX-B parameters, $\tau_{F S} \sim 3 \mu \mathrm{s}$.

An established method of reducing losses in open-ended systems is by the use of multiple magnetic mirrors.22,23 The effect can be understood as a friction on the ions streaming towards the ends resulting from an interaction with trapped particles in the mirror cells. The optimum confinement occurs when the classical ion mean free path, $\lambda$, is comparable to the cell length, $\ell$, in which case

$$
\tau_{M M} \sim \frac{M^{2} L_{1 / 2}}{\lambda v_{i}} \text { for } \ell \sim \lambda
$$

A linear array of field-reversed cells as sketched in Fig. 6 creates a multiple-mirror configuration more complex than that assumed in deriving the above formula for $\tau_{M M}$. However, the similarity in the open tield lines for the two cases leads to the expectation of a simllar confinement of ions along these field lines. There are possible additional benefits because field lines near the separatrix pass through a localized field minimum at the end of each CT plasma cell. The resulting non-adiabatic ion trajectories might enhance the effect of multiple mirrors although calculations have not yet been performed to support this conjecture.

Using FRX-B parameters the mean free path for ions on the separatrix is estimated knowing the density and temperature. For $\mathrm{n} \sim 2 \times 10^{15} \mathrm{~cm}^{-3}, \mathrm{~T}_{\mathbf{i}} \sim 200$ $\mathrm{eV}$, the mean free path $\lambda$ is $\sim 33 \mathrm{~cm}$. With this $\lambda$ the optimum multiple-mirror confinement is obtained with a periodic length of $33 \mathrm{~cm}$ (15 cells in the case of the Scylla IV-P 5-m coil). In the proposed experiment, the number of cells defined by the periodicity of the applied field mirrors will be adjustable from 1 to 15 since it is not certain what effect the cell length will have on the stabilty. Depending on the number of cells that can be stably formed the estimated confinement for $200 \mathrm{eV}$ ions on the open field lines is: 


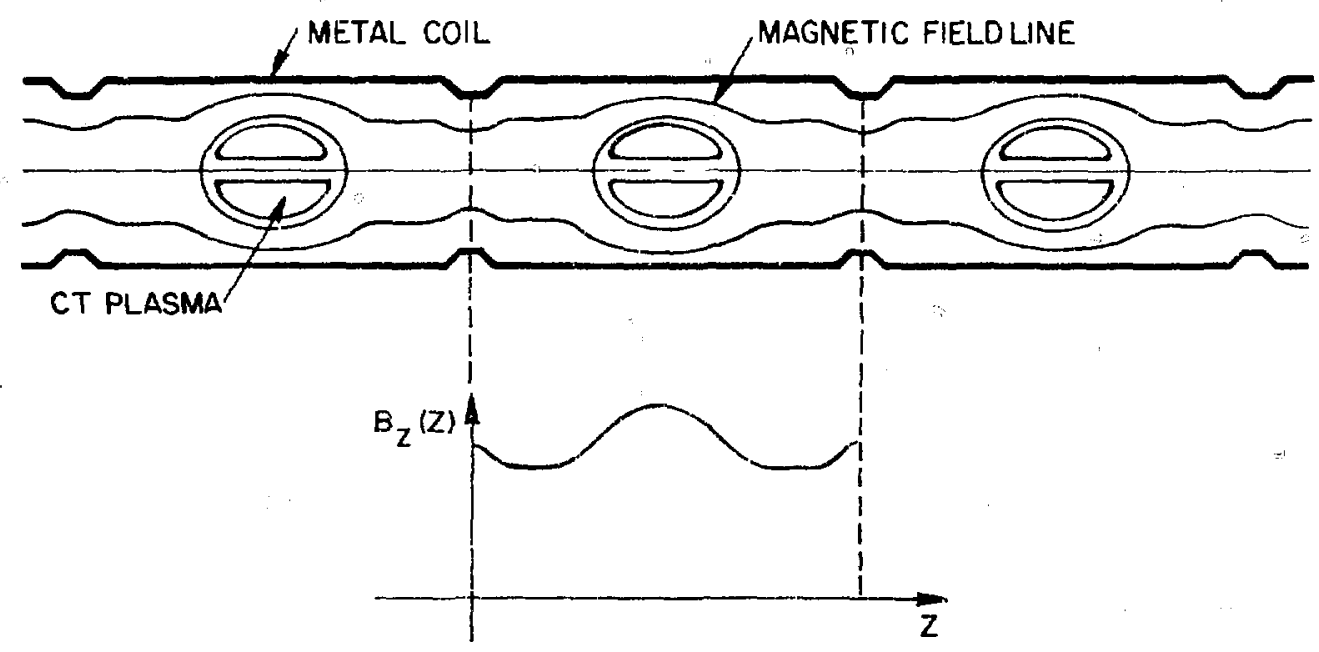

Fig. 6 .

Sketch of magnetic fields in the vicinity of several field-reversed configuration (FRC) plasma cells showing the origin of multiple magnetic
mirrors.

$$
\begin{array}{ll}
15 \text { cells } & \tau_{M M}=\frac{3(1.2)^{2}(250)^{2}}{(33) 1.4 \times 10^{7}}=133 \text { usec. } \\
5 \text { cells } & T_{M M}=\frac{(1.2)^{2}(250)^{2}}{(100) 1.4 \times 10^{7}}=64 \text { usec. }
\end{array}
$$

It should be polnted out that the time $\tau_{\mathbb{M}}$ characterizes the ion particle and energy loss rate in the absence of axial electron thermal conduction. If the electrons conduct heat to a cold boundary at the ends of the 5-m coil they represent an additional heat loss process through equilibration with the ions. The characteristic ion cooling time would be about 75 usec for FRX-B parameters. Although multiple-mirror effects" would still be beneficial in the presence of electron thermal conduction, the experiment is improved by avolding 
this conduction. It is assumed here, as in the Livermore Mirror Program, that classical electron thermal conduction is avoided if the material boundary on open field lines acts to absorb the exhaust plasma without significant emission of secondary electrons or ablated plasma. For the conditions in this experiment where one-half of the plasma energy $(\sim 3 \mathrm{~kJ})$ is released in about 100 usec, an area of about $0.4 \mathrm{~m}^{2}$ at each end of the experiment is large enough to limit the temperature excursion well below the melting point of a number of materials including aluminum oxide and stainless steel.24,25 Thus it appears possible to test multiple-mirror confinement without the added complexity of axial thermal conduction.

\section{Future Plans}

The above descriptions of FRX-C and the Scylla IV-P Multiple-Cejl Experiment emphasize the issues thought to be most important in the first year of operation. There are of course many other issues which must be studied befcre selecting an attractive CT configuration and developing a CT proof of principle experiment in the $80^{\circ} \mathrm{s}$. Data for the basic choice between single and multiple cells comes from the Scylla IV-P Multiple-Cell Experiment and from experiments in the CTX facllity with the FRX-C and the magnetized gun as plasina sources.

The first issue to be addressed, after FRX-C has been successfully operated is the translation and trapping of the CT plasma. This wtil be investigated by adding asymmetric mirror flelds to FRX $-C$ in order to move the plasma from the theta-pinch coil through a guide field and into the steady-state field of the large CTX vacuum vessel. Experiments with asymmetric mirrors on FRX-A have successfully demonstrated plasma translation out of the theta-pinch coil and FRX-B experiments in FY-80 will include an external quasi-steady coil to permit further observations of translation. Trapping experiments will also be done to the extent possible with the limited lifetime of the FRX-B plasma.

The next major issue to address is heating. By approximately the end of FY-82, it will be possible to evaluate the magnetized plasma gun and FRX-C sources on CTX, and to begin heating experiments. In the meantime, a number of preliminary heating experiments and analytic studies of the various heating 
alternatives are needed and will be carried out to the extent possible within budgetary limitations. Heating experiments in the present FRX-A and FRX-B devices are restricted to the techniques capable of producing high power for a short time because of the relatively short plasma lifetime.. Some examples of methods being considered are magneto-acoustic heating or adiabatic compression possibly with a translated toroid using a passive liner.

Longer energy confinement times are a major goal of LASL's CT research. When characteristic plasma times of about 1 ms are achieved, the use of neutral beam heating becomes viable. A preliminary study of the scientific and engineering feasibility is urgently needed in FY-80 to determine the implications of neutral beam heating in terms of costs, scheduling, hardware options, and modifications to the CTX facility. This first step is necessary to keep pace with the experimental program plan (Apppendix A) almed at starting heating studies towards the end of FY-82. 


\section{(3.120) ENGINEERING DESCRIPTION}

\section{The FRX-C Experiment}

The geometrical arrangement of $\mathrm{FRX}-\mathrm{C}$ was shown in Fig. 5. A coll diameter of $0.45 \mathrm{~m}$ and length of $1.5 \mathrm{~m}$ is compatible with commercially available $0.4-\mathrm{m}$ i.d. quartz discharge tubes. Driven mirror coils at each end will increase the effective coil length to approximately $1.8 \mathrm{~m}$. The eventual use of $\mathrm{FRX}-\mathrm{C}$ as a plasma injection source for CTX (see Appendix A) makes it desirable to locate FRX-C adjacent to and on a centerline with the CTX vacuum tank. Limited space led to the particular design shown in Fig. 5. The collector plates and high voltage capacitor bank are located above the discharge coil to improve diagnostic access and to facilitate connections with the pulse charge circuitry and trigger circults located overhead on a presently existing deck. The magnetic field strength of $10 \mathrm{kG}$ in $\mathrm{FRX}-\mathrm{C}$ presents no special mechanical problems in the design of discharge coil, transition plates, and collector plates.

The electrical arrangement, designed to generate the high voltage needed according to the scaling laws developed in Appendix D, uses methods previous $1 \mathrm{y}$ developed in the LASL Implosion Heating Experiment (IHX) and Staged Theta Pinch Experiment (STP). Many components left from IHX and STP have been utilized in the design of FRX-C to save time and money. Examples are the collector plates, main-bank spark-gap switches, pulse charge clrcuit components, and trigger circuit components.

Figure 7 indicates in a block diagram the FRX-C electrical circuit. Not shown are the bias bank, preionization bank, and mirror coil banks. Pulse charging is used in the main bank of $240.75-\mu f$ capacitors to obtain higher voltage ( $125 \mathrm{kV})$ and higher energy density $(5.9 \mathrm{~kJ} /$ capacitor) than is possible with dc charging. An example of the high voltage circuitry to be employed on FRX-C is shown in Fig. 8, a photograph of the IHX experiment. In that experiment four collector plates and feed slots were used on the 0.4-m diameter coil. The coil and main bank are in the center of the photograph. Pulse charge maxi-marx circuits are in the foreground on the right and left of the photograph. 


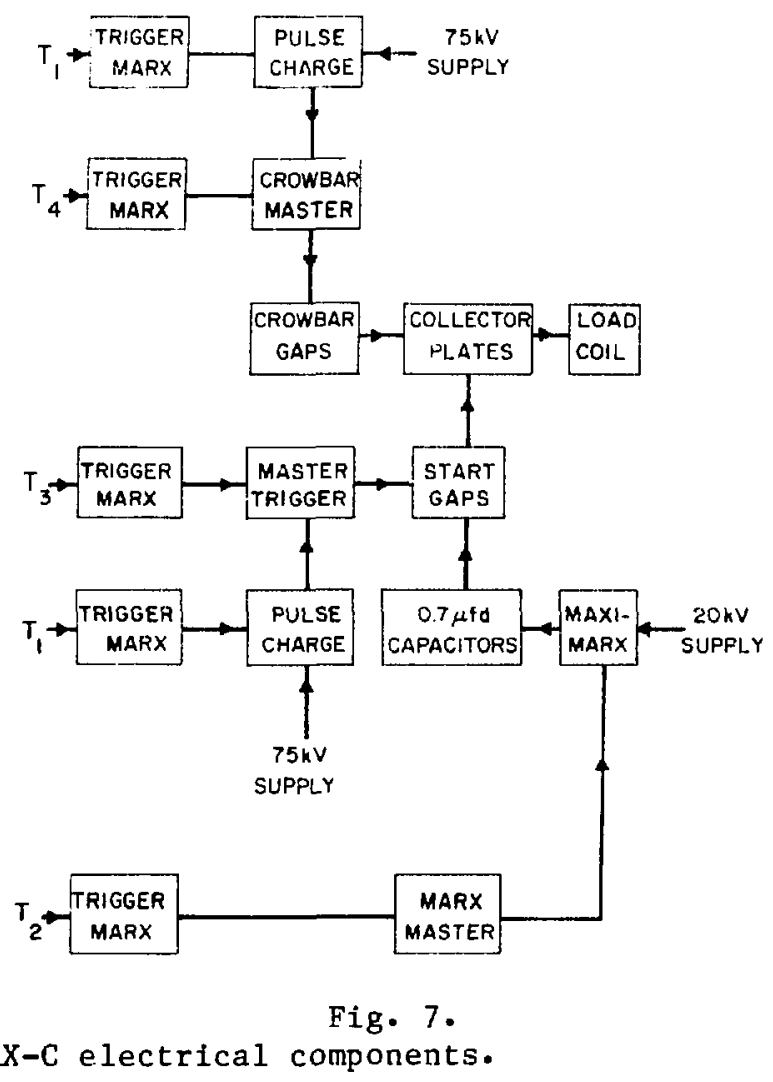

Computer calculations of the current or magnetic field waveform for FRX $-C$ are shown in Fig. 9 taking realistic account of the various source inductances and spark gap resistance. The calculation also includes the effect of reduced coil inductance at early times resulting from a discharge tube full of preionized plasma and shows the non-sinusoidal current that results from the time varying plasma inductance. The relatively rapid current risetime, compared to the estimate in Table I based on a sinusoidal waveform, will have a beneficial effect in further reducing the plasma expansion during the time interval when the field is reversed. As discussed in Appendix $D$, the best field-reversed theta-pinch operation is observed when this reversal time is minimized, since the trapped flux and plasma size relative to the metal wall are maximized.

No unusual problems are anticipated in the vacuum system, computer control system, data acquisition system, or other aspects of the experiment. As discussed in Appendix A, the experiment will share the CTX-facility control system, screen room, and experimental area (previously the STP area) with the 


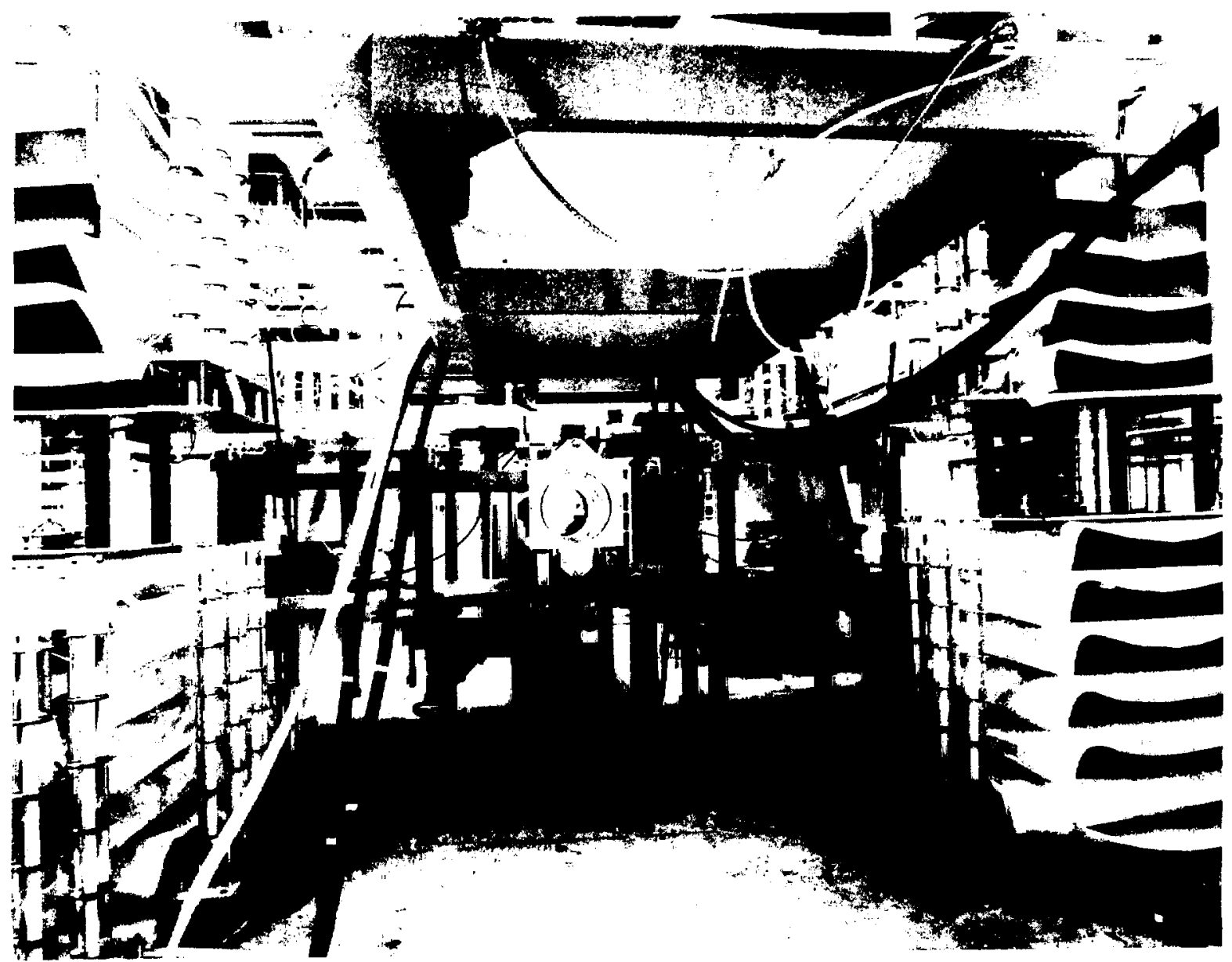

Fig. 8.

Photograph of the IHX experiment showing components similar to those needed for FRX-C.

magnetized Marshall plasma gun experiment. One element of the electrical circult, the crowbar switch, requires some development and for this purpose a 1/4-scale electrical prototype is planned. Based on previous LASL experience with similar high-voltage experiments, reasonable confidence exists that FRX- $\dot{C}$ can be constructed and operated on schedule. 


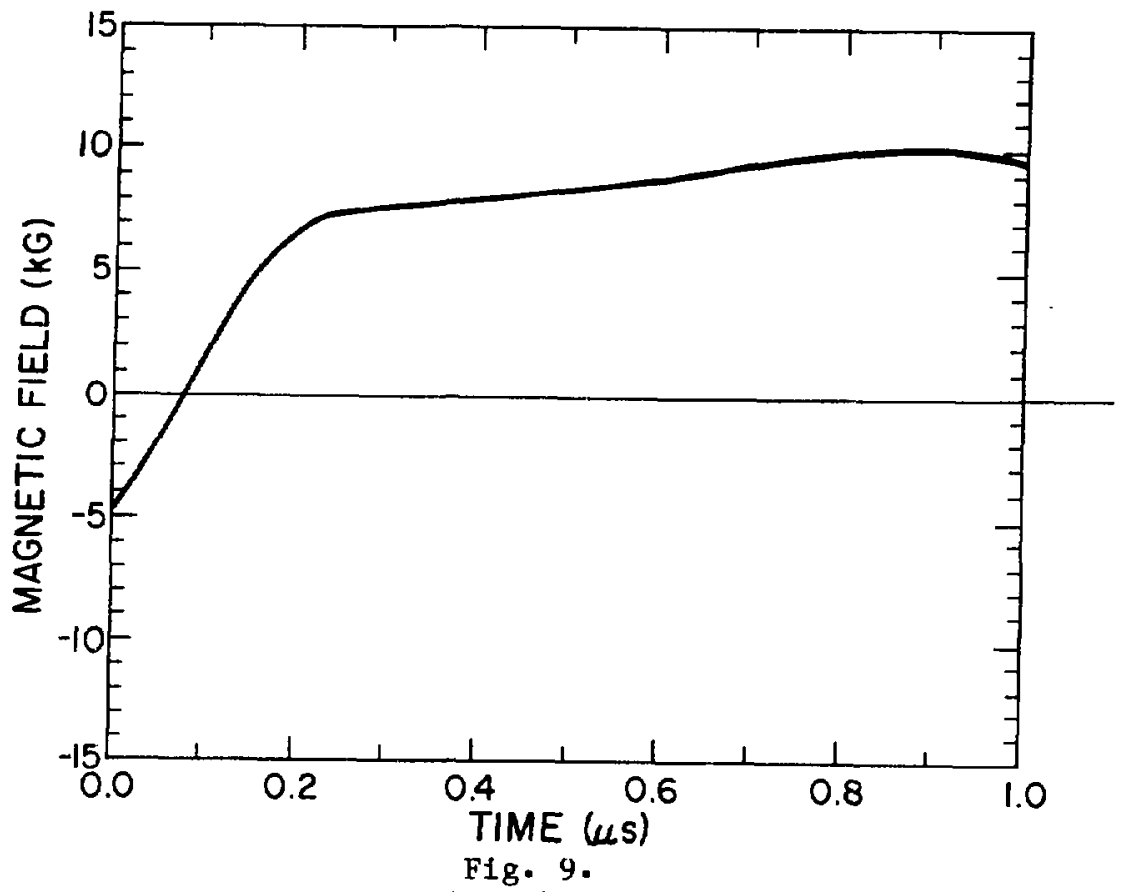

Calculated magnetic field waveform for the FRX-C experiment.

The Scylla IV-P Multiple-Cell Experiment

By modifying the existing Scylla IV-P theta pinch to generate the same magnetic field waveform in the same diameter coil as FRX-B, it should be possible to generate a linear array of CT's with known single-cell properties. Figure 10 shows the 5-m long coil arrangement. By the use of positionable coil sections, the number of cells formed as the result of tearing modes induced by coil shaping can be varied. It is convenient to vary the number of cells from one to fifteen (five cells correspond to five ret ilcas of FRX-B) using the present mechanical design of the Scylla IV-P collector plate and coil system.

The Scylla IV-P theta pinch has previously been used in detalled studies of linear theta-pinch physics and end-stoppering techniques described elsewhere. It was proposed and approved in FY-74, and construction was completed in FY-76. The device is a conventional theta pinch of 5-m length powered by a 60-kV, 2-MJ capacitor bank of the Scyllac type.

The Scylla IV-P facility occuples the basement of LASL Building SM 105, popularly known as the "Pit," which is a room 50-feet square with a 25-foot celling. Figure 11 shows an elevation plan view of the device. The 


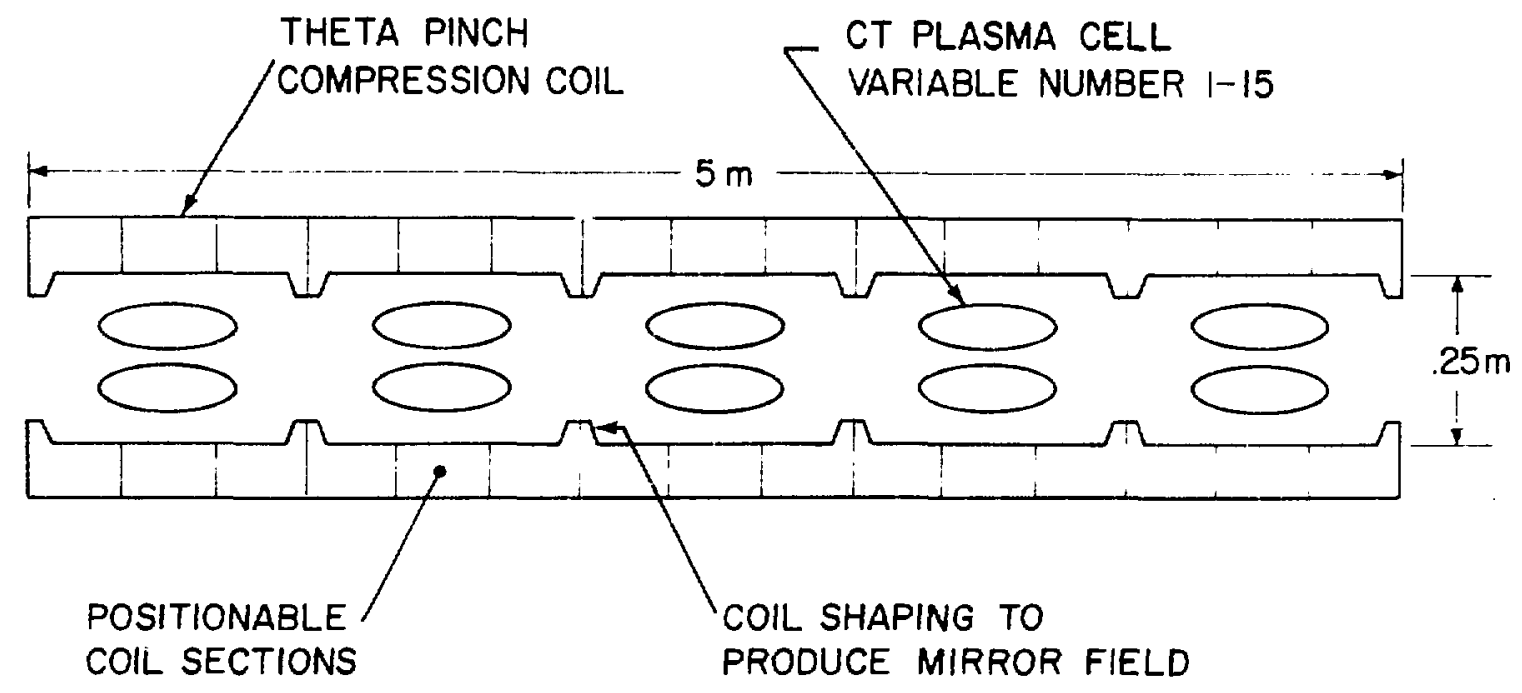

\section{MULTIPLE-CELL EXPERIMENT}

Fig. 10.

Magnetic compression coil arrangement for the Scylla IV-P multiple-cell experiment.

capacitor-spark-gap units of the energy storage system are arranged in clusters on a three-level platform structure. This layout facilitates trouble shooting and bank maintenance. The collector plates and 5-m long compression coll are situated on the middle platform level which also accomodates the majority of the plasma diagnostic systens. Figure 12 shows a photograph of the theta-pinch "front end," including the diagnostic equipment avallable in the Scylla IV-P facility. Diagnostics include Thomson scattering, ruby laser and coupled-cavity interferometers, neutron detectors, spectrometers, optical plasma position detectors, streak and framing cameras, compensated diamagnetic loops, and internal magnetic field pressure probes. 


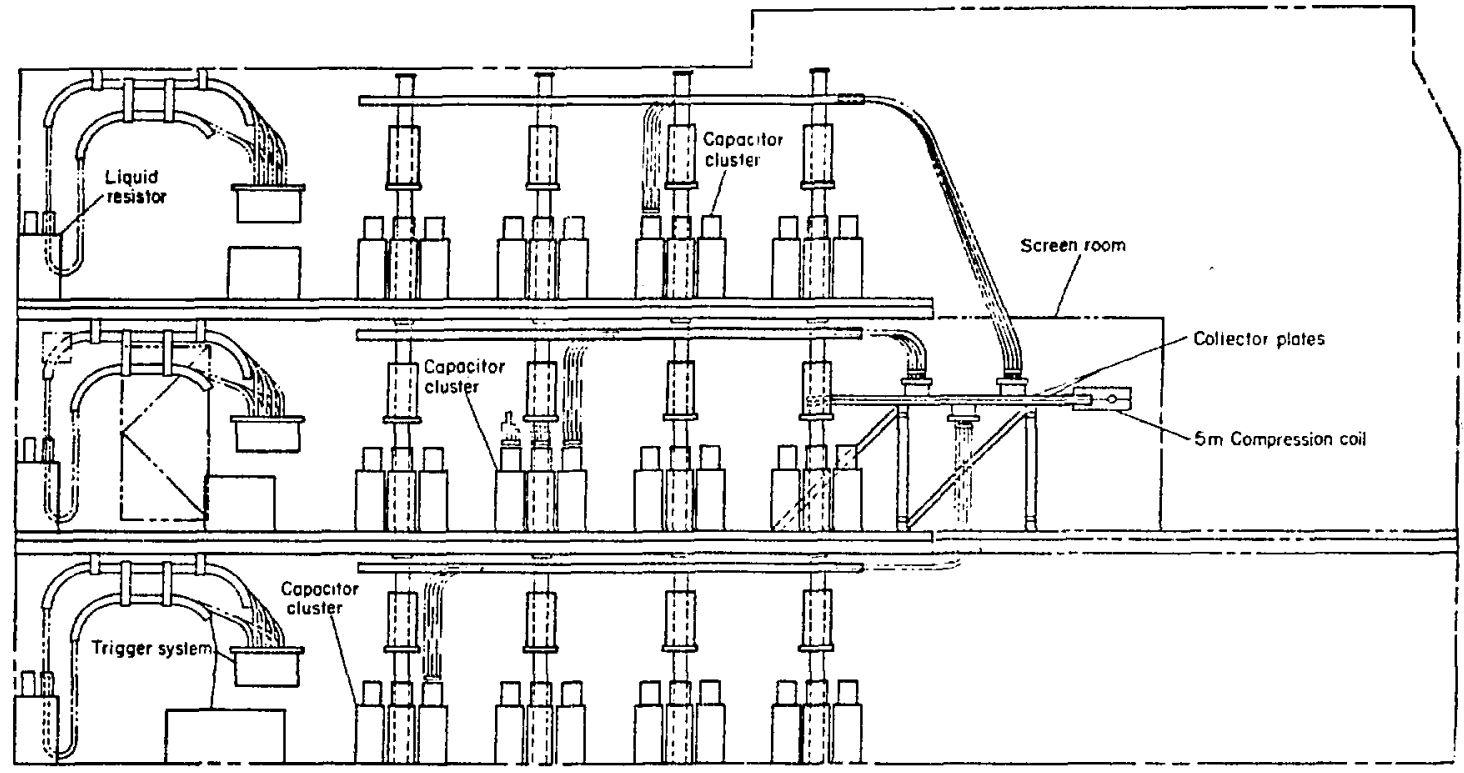

Scylla $\mathbb{\nabla}-\mathrm{P}$ Elevation View

Fig. 11 .

Elevation view of the Scylla IV-P capacitor bank arrangement.

The Scylla IV-P theta pinch was the first CTR experiment at Los Alamos to be completely computer controlled. The computer hardware, which is contained within a 10-foot by 40-foot air conditioned screen room, consists of a prime 300 with $32 \mathrm{~K}$, 16-bit words, a 3-million word disk, a Tektronix display terminal, a Versatec printer-plotter, and a CAMAC system to interface to the Scylla IV-P device. The computer is used for both machine operation and data acquisition. A total of 140 computerized data acquisition channels are presently available in Scylla IV-P. Data can be analyzed "on line" by the Prime 300 computer or shipped via a serial link to a PDP-10 for subsequent analysis.

The Scylla IV-P device has been operated continually for the past three years without an overhaul of the capacitor bank spark gaps. In order to carry out the proposed experiment refurbishing of a portion of the capacitors is required. Since a peak confining field strength of $9 \mathrm{kG}$ will be used, about $25 \%$ of the existing Scylla IV-P energy storage system will be required to generate the field. An initial bias field of $-3 \mathrm{kG}$ (opposite to the direction 


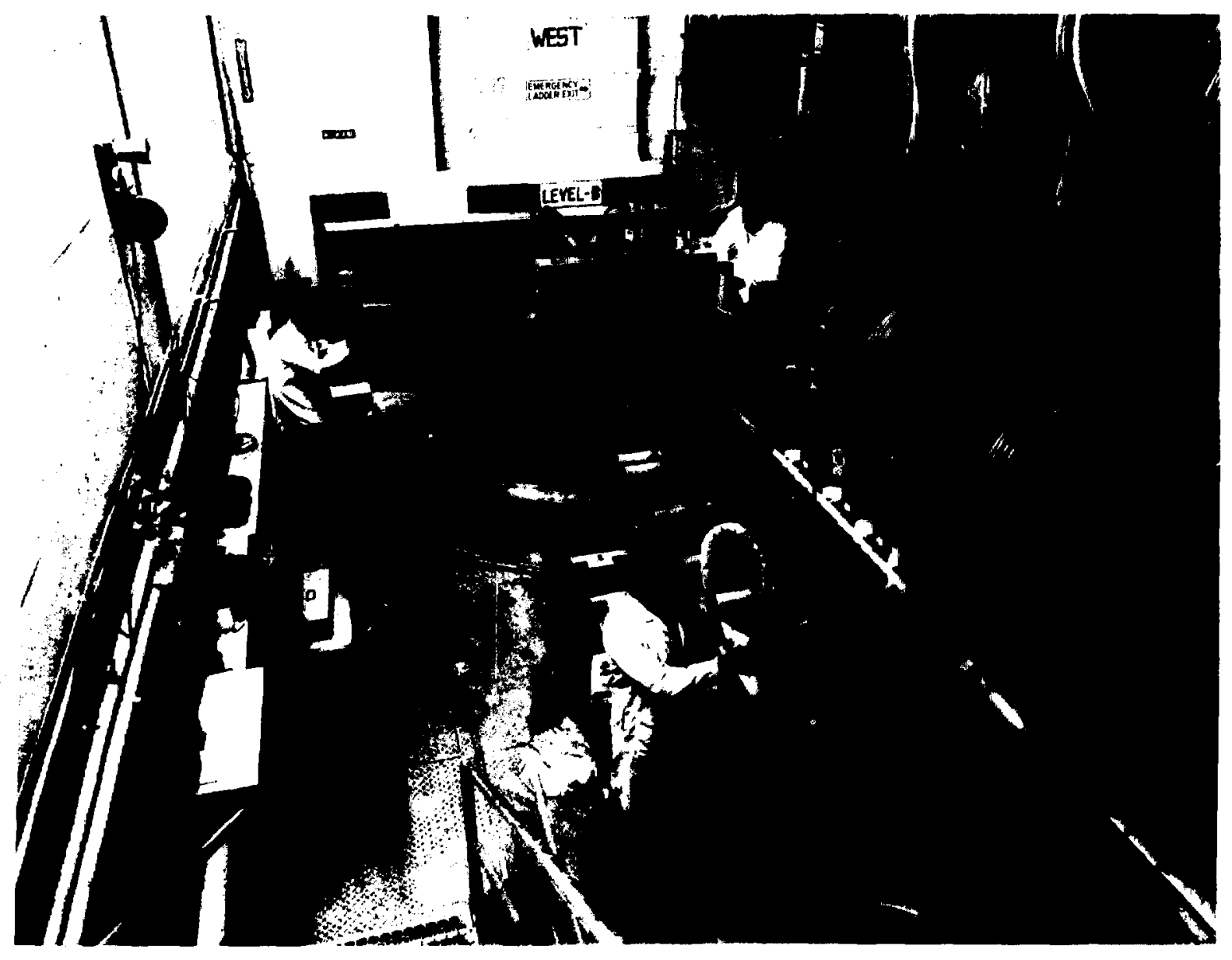

Fig. 12.

Photograph of Scylla IV-P showing the collector plates, the compression coil, and various Instruments on the diagnostics platform.

of the main fleld) will be generated with high energy density, low voltage, ignitron switched capacitors in a bank similar to the bias field bank developed for $2 \mathrm{~T}-40$.

The experimental layout of the multiple-cell device will basically be the same as that illustrated in Fig. 11. The bottom floor level will contain only the bias bank capacitors (45-185 $\mu \mathrm{F}$ unfts) and associated size $\mathrm{D}$ ignitron switches. The main bank capacitors (145-1.8 $\mu \mathrm{F}$ units) will be located on the middle and upper platform levels. The front of the middle platform level, 
which accommodates the collector plates and most of the plasma diagnostic systems, will not be changed from the present configuration.

Using the required experimental dimensions and magnetic field strengths, the necessary energy storage systems and associated electrical parameters were obtained using the SCEPTRE circuit analysis program on the Lawrence Livermore Laboratory MFECCA computer. Tables II and III give respectively the resultant main bank and bias bank operating parameters. Theta-pinch preionization will be used as in the present Scylla IV-P and FRX-B experiments. Plasma parameters for FRX-B were given in Table $I$.

The needed exiansion chambers to maintain an ambipolar sheath and avoid classical electron thermal conduction are modest in size. For stainless steel it appears that cylinders at each end of the discharge tube $0.3 \mathrm{~m}$ in diameter and $0.3 \mathrm{~m}$ in length provide sufficient area to absorb the energy without

TABLE II.

Multiple-Cel1 Main Bank Parameters

$\frac{\text { Parameter }}{\mathrm{W}_{\text {bank }}(60 \mathrm{kV})}$
$\mathrm{C}$ )
$\mathrm{V}_{\text {bank }}$ (operating)
$\mathrm{L}_{\text {coil }}(\max )$
$\mathrm{L}_{\text {sgurce }}$
$\tau$ qu
$\mathrm{L} / \mathrm{R}$
$\mathrm{B}_{\max }(40 \mathrm{kV})$

$$
\begin{aligned}
& \frac{\text { Value }}{0.5 \mathrm{MJ}} \\
& 268 \mu \mathrm{F}(145-1.85 \mu \mathrm{F}) \\
& 40 \mathrm{kV} \\
& 12.3 \mathrm{nH} \\
& 2.6 \mathrm{nH} \\
& 2.8 \mu \mathrm{s} \\
& 100 \mu \mathrm{s} \\
& 9 \mathrm{KG}
\end{aligned}
$$

TABLE III.

Multiple-Cell Bias Bank Parameters

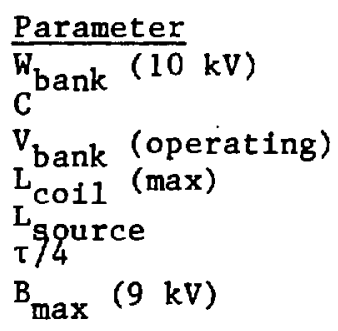

Value

$\overline{0.4 \mathrm{MJ}}$

$8235 \mu \mathrm{F}(45-185 \mu \mathrm{F})$

$9 \mathrm{kV}$

$12.3 \mathrm{hH}$

$360 \mathrm{hH}$

90 us

$-3 k G$ 
ablation or secondary electron emission. Because of the finite field diffusion time in stainless steel, a weak quasi-steady magnetic field introduced before the pulsed discharge is necessary to guide exhaust plasma onto the metal surfaces. Another possibility being considered is the use of ceramic aluminum oxide cylinders which obviates the need for the quasi-steady field.

\section{Plasma Diagnostics}

Both FRX-C and the multiple-cell experiment are logical next step experiments beyond FRX-B. Consequently, each should include most of the diagnostics used on FRX-B for comparison. Thus excluded flux loops, quartz UV carbon spectroscopy, Thoms on scattering, side-on and end-on framing and streak camera pictures, and magnetic probes down the center are planned for the new experiments. These measurements should ideally be performed on all cel.ts of the multiple-cell experiment but a reasonable compromise is the center, intermediate, and end cells of one side of the experiment. Carbon is expected to be completely ionized in the interesting regions of $F R X-C$, and the $U V$ spectroscopy will not be as useful as it is on FRX-A. Framing pictures may not be as useful in observing the onset of the rotational instability for the multiple-cell experiment since the instability may not occur in all cells at the same time, and light from varied angular positions of the other cells will decrease the contrast of light from any one cell. However, the initial hole in the center might still be visible and careful focusing at various places in the tube may make it possible to observe individual cells from the end. Visible light and excluded flux loops can be used to study the translation of FRX-C plasma into CTX. The use of the above mentioned diagnostics on FRX-B has been discussed previously ${ }^{8}$ and since the applications here are so similar, little further discussion of these diagnostics is needed.

FRX-C is designed to reach higher electron and ion temperatures than FRX-B, and UV carbon spectroscopy may be lost as a valuable diagnostic, but neutron measurements may give valuable information about the deuterium ions. If FRX-C achieves ion temperatures near $1000 \mathrm{eV}$ or above and lifetimes of 100 us or longer, then well over $10^{8}$ neutrons will be produced, which will make neutron emission a useful ion temperature diagnostic. In this experiment one expects the highest temperature and density to occur near the field null 
radius, and because of the strong density and temperature dependence of the neutron production rate most of the neutrons will come from a fairly narrow region around this field null. If Thomson scattering is used to measure the density in this region, the rate of neutron production could be used to measure the ion temperature provided the ions have a thermal distribution at sufficiently high temperature. If ion temperatures above $1000 \mathrm{eV}$ are achieved, it may be possible to measure plasma rotational speeds by taking advantage of a resonant transmission of neutrons which occurs in liquid oxygen. 26 This resonance is fairly sharp with the $2.5-\mathrm{MeV}$ DD neutrons being on the steep part of the absorption curve. Two balanced detectors looking through liquid air positioned at the bottom of the machine but on opposite sides will see a difference signal due to energy shifts tokard and away from the transmission maximum. The difference signal and the total signal could then be used to measure the rotation of the plasma. After the neutron production rate is known, a more careful look at this possibility will be necessary to determine if the difference signal will be above the noise. Side-on Faraday rotation measurements would be valuable in FRX-C to check for the unexpected but possible existence of $B_{t}$. To do this ports through the coil would need to be added with windows that transmit $10.6 \mu \mathrm{m}$ radiation. The long wavelength is necessary so that small $\mathrm{B}_{t}$ can be detected.

In the multiple-cell experiment it will be important to measure particle and energy confinement times in order to understand these confinement times as a furction of the number of cells as well as for comparison with FRX-B. This can be done through the use of many excluded flux loops, multiposition Thomson scattering and end-on interferometry. In this experiment extensive use of computer data acquisition and analysis will be necessary since many cells will be studied. It seems that approximately $32 \mathrm{flux}$ loops on one half of the machine could be used to study the size and shape of the cells and their energy per unit length. These exluded flux loops could give the energy per unit length as a function of axial position, the energy decay time for each ce11, and the overall energy decay time. In this long experiment the plasma at the ends will introduce less errors than in the single cell experiments, and reasonably accurate overall particle inventory decay times can be measured using end-on two-time holographic or multibeam interferometry. Thomson scattering should be done in at least three cells so the $T_{e}$ and $n$ could be measured as a function of axial position. This might be done using one laser 
and beam splitter which would simplify the data acquisition in that one gate time could be used for ali three stations. It is important here to use Thomson scattering for at least relative density information from cell to cell. The one laser concept helps to eliminate some errors in this part of the measurement.

Proper use of the diagnostlcs mentioned should give sufficient information for a good understanding of the physics of these two experiments. Most of the diagnostics are standard at Los Alamos and could be fmplemented with little difficulty. 
The FRX-C Experiment

Costs are estimated assuming that all design, the majority of construction, and all diagnostics and operations are carried out by permanent LASL manpower. As mentioned above, considerable savings in cost and time are realfzed by using available components from IHX and STP. Major procurement is largest in the first fiscal year and is itemized in Table IV. The total cost given in Table $V$ is based on the assumption that the experiment is constructed in approximately one year and operated for approximately two years. Except for continuing diagnostic hardware costs and other small items, 'the only anticipated major procurement items after FY-80 are a transition coil and vacuum components to link FRX-C to the CTX vacuum vessel (see Appendix A). Manpower requirements are summarized below in Table VII.

TABLE IV.

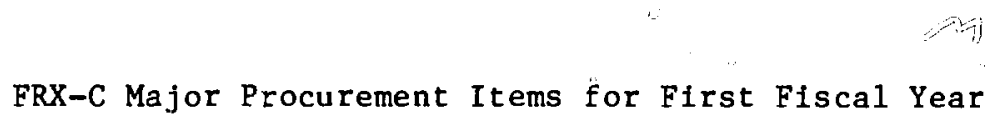

Item

Electronics for control and data acquisition Fiscal Year

Diagnostics hardware

Capacitor bank components

70

Quartz discharge tubes

35

Vacuum components

25

Mechanical structure

30

Compression coil

25

Miscellaneous 
TABLE V.

Total FRX C Costs (K\$)

\begin{tabular}{|c|c|c|c|c|}
\hline . & $\underline{F Y-80}$ & $\underline{F Y-81}$ & $\underline{F Y-82}$ & Total \\
\hline Manpower (permanent) & 1160 & 1410 & 1840 & 4410 \\
\hline Construction labor (temporary) & 85 & 0 & 0 & 85 \\
\hline Major procurement & $\underline{290}$ & $\underline{150}$ & $\underline{200}$ & $\underline{640}$ \\
\hline Subtotal & 1535 & 1560 & 2040 & 5135 \\
\hline Capital equipment & $\underline{300}$ & $\underline{150}$ & 150 & $\underline{600}$ \\
\hline Total & 1835 & 1710 & 2190 & 5735 \\
\hline
\end{tabular}

The Scylla IV-P Multiple-Ce1l Experiment

Costs are based on the assumption that approximately one year is required for modification of the Scylla IV-P facilfty, including main-bank refurbishing, and at least one year is needed for operation. The totals are given in Table $V I$ in terms of first and second fiscal years following approval. In an aggressive program the work would be started in FY-80; in a modest pace program the funding would be delayed until FY-81.

TABLE VI.

Total Scylla $\perp V-P$ Multiple-Cell Experiment Costs (K\$)

$\begin{array}{cccc} & \text { First fiscal year } & \text { Second fiscal year } & \frac{\text { Total }}{1360} \\ \text { Manpower (permanent) } & 520 & 840 & 80 \\ \text { Technician labor (temporary) } & 80 & 0 & \underline{290} \\ \text { Major procurement } & \underline{240} & \underline{50} & 1730 \\ \text { Sub-Total } & 840 & 890 & \underline{200} \\ \text { Capital equipment } & \underline{120} & \underline{80} & 1930 \mathrm{KS}\end{array}$




\section{(3.140) MILESTONES}

The FRX-C Experiment

\begin{tabular}{|c|c|}
\hline $\begin{array}{l}\text { August, } 1979 \\
\text { September, } 1979\end{array}$ & $\begin{array}{l}\text { Proposal submitted to DOE } \\
\text { DOE dectsion }\end{array}$ \\
\hline December, 1979 & Complete construction on FRX-C electrical prototype \\
\hline February, 1980 & Prototype evaluated and final design completed of FRX-C \\
\hline August, 1980 & $\begin{array}{l}\text { Complete construction and begin electrical check-out of } \\
\text { FRX-C }\end{array}$ \\
\hline October, 1980 & Begin operation of FRX-C \\
\hline August, 1981 & $\begin{array}{l}\text { Evaluate FRX-C scaling and decide whether to begin } \\
\text { modifications for translation and trapping experiments }\end{array}$ \\
\hline November, 1981 & $\begin{array}{l}\text { Assuming a favorable August ' } 81 \text { decision, } \\
\text { begin injection studies in CTX }\end{array}$ \\
\hline
\end{tabular}

The Scy1la IV-P Multiple-Ce11 Experiment

$\begin{array}{lll}\text { First fiscal year } & \begin{array}{l}\text { October } \\ \text { January }\end{array} & \begin{array}{l}\text { Begin main-bank refurbishing } \\ \text { Finish design of modifications; place } \\ \text { order for new coll }\end{array} \\ \text { March } & \text { Finish main-bank refurbishing } \\ \text { June } & \text { Finish construction; begin electrical } \\ & \text { check-out } \\ & \text { September } & \begin{array}{l}\text { Begin operation of experiment } \\ \text { March }\end{array} \\ \text { Second fiscal year } & \text { diagnostics } \\ & \text { September } & \text { Evaluate multiple-cell ct confinement }\end{array}$


The FRX-C Experiment

Mechanical engineering

\begin{tabular}{|c|c|c|}
\hline FY-80 & FY-81 & $F Y-82$ \\
\hline 1.5 & 1.0 & 1.0 \\
\hline 1.0 & 1.0 & 1.0 \\
\hline 1.0 & 1.0 & 1.0 \\
\hline 4.5 & 8.0 & 10.0 \\
\hline 13.0 & 10.0 & 12.0 \\
\hline 21.0 & 21.0 & 25.0 \\
\hline
\end{tabular}

Electrical engineering

Computer support

Experimental staff

cientists

Technicians

The Scylla IV-P Multiple-Cell Experiment

$\begin{array}{lcc}\text { Mechanical engineering } & 0.5 & 0.25 \\ \text { Electrical engineering } & 0.5 & 0.25 \\ \text { Computer support } & 0.5 & 1.0 \\ \text { Experimental staff } & & \\ \quad \text { Scientists } & 2.5 & 4.5 \\ \text { Technicians } & \underline{5.0} & \underline{7.0}\end{array}$




\section{REFERENCES}

1. N. C. Christofilos, Progress in Nuclear Energy; Series XI-P lasma Physics and Controlled Nuclear Fusion 1, 576 (Pergamon Press, Oxford, 1959).

2. R. N. Sudan and E. Ott, Phys. Rev. Lett. 33, 355 (1974); S. Humphries, Plasma Phys. 17, 973 (1975); H. H. Fleishmann, Ann. N. Y. Acad. Sci. $\underline{251}, 472$ (1975).

3. J. Golden, C. A. Kapetanakos, R. A. Mahaffey, J. A. Pasour, and S. J. Marsh, Bull. APS 23, 815 (1978).

4. L. D. Pearlstein, et al., Seventh International Conference on Plasma Physics and Controlled Nuclear Fusion Research, Innsbruck, 1978 (IAEA, Vienna, to be published); R. F. Post, UCRL-81586 (1978).

5. W. B. Jones and R. D. Miller, Phys. Fluids $\underline{11}, 1550$ (1968).

6. R. Turner, Phys. Fluids 13,, 2398 (1970).

7. D. R. Wells, et al., Phys. Rev. Lett. 33, 1203 (1974).

8. R. K. Linford et al., Proc. of the Seventh IAEA Conference on Plasma Physics and Controlled Nuclear Fusion, Innsbruck, 1978 (IAEA, Vienna, to be published).

9. G. C. Goldenbaum, Y. P. Chong, G. Hart, and J. H. Irby, Paper 3E8, Proc. of 1979 IEEE International Conference on Plasma Science, June 4-8, (Montreal, 1979).

10. H. Alfvén, et al., J. Nucl. Energy, Part C: Pl. Phys. 1, 116 (1960).

11. J. D. Sethian, K. A. Gerber, D. N. Spector, A. E. Robson, Phys. Rev. Lett. 41, 798 (1978).

12. S. Robertson and A. F1sher, Paper 2Q5, Proc. of 1979 IEEE International Conference on Plasma Science, June 4-8, (Montreal, 1979).

13. M. N. Bussac, H. P. Furth, M. Okabayashi, M. N. Rosenbluth, and A. M. Todd,

Proc. Seventh IAEA Conference on Plasma Physics and Controlled Nuclear Fusion, Innsbruck, 1978 (IAEA, Vienna, to be pub1ished) paper $\mathrm{CN}-37-\mathrm{X}-1$.

14. P. J. Turch1 and A. E. Robson, Proceedings of the S1xth Symposium on Engineering Problems in Fusion Research, San Diego, Calif., 1975, p. 983.

15. G. A. Carlson et al., LLL Report UCRL-52467 (1978).

16. A. C. Smith et al., Pacific Gas and Electric Company Report 78FuS-1 (1978). 
17. C. E. Seyler, W. Grossman, and L. C. Steinhauer, Comments Plasma Phys. Cont. Fusion 4 , 21 (1978).

18. W. T. Armstrong, R. K. Linford, J. Lipson, D. A. Platts, and E. G. Sherwood, Los Alamos Scientific Laboratory report LA-UR-1160 (1979).

19. W. T. Armstrong, R. K. Linford, J. Lipson, D. A. Platts, and E. G. Sheiwood, submitted to Ninth European Conference on Controlled Fusion and Plasma Physics, Culham (1979).

20. S. Hamasakf, Conference Record of 1979 IEEE International Conference on Plasma Science, p. 143.

21. R. K. Linford, private communication.

22. A. Makhijani, A. J. Lichtenberg, M. A. Lieberman, and B. Grant Logan, Phys. Fluids 17,1291 (1974).

23. V. V. Mirnov and D. D. Ryutov, Nuc1. Fusion 12, (1972).

24. R. H. Lovberg, in Plasma Diagnostic Techniques (R. H. Huddlestone and S. L. Leonard, Eds., Academic Press, New York, 1965) p. 69.

25. G. E. Gryczkowski, R. A. Krakowski, L. C. Steinhauer, and J. Zumdieck, Third ANS Meeting on the Technology of Controlled Nuclear Fusion, Santa $\mathrm{Fe}$, New Mexico, May 9-11, 1978 (also USDOE Report LA-UR-78-1470 (1978)).

26. P. H. Stelson and C. F. Barnett, Bull. APS 23, 882 (2678). 
APPENDIX A--PROVISIONAL LASL COMPACT TORUS PROGRAM PLAN May, 1979

R. K. Linford, A. R. Sherwood, and R. E. Siemon

\section{Introduction}

Several reactor schemes have been proposed $1,2,3$ based on the Compact Torus (CT) concept which appear to have significant advantages over conventional tokamak reactor plans. The experimental program Plan described in this document addresses many of the important issues that must be resolved before the viability of these reactor schemes can be properly assessed.

The term CT refors to a toroidal plasma configuration with no material other than plasma in the "hole" of the torus. Thus it excludes toroidal systems such as the tokamak, reversed-field pinch (RFP), and ELMO bumpy torus (EBT). The magnetic fields in a CT plasma may be supported by high energy beams, as in Astron-like devices, or by thermal plasma currents. The Astron-ring configurations are being studied extensively in other laboratories, so this Program Plan is restricted to the study of $\mathrm{CT}^{\circ}$ 's supported by thermal plasma current. This latter class of $\mathrm{CT}^{\prime \prime} \mathrm{s}$ can also be separated into two subclasses: with toroidal field, $B_{t}$, and without toroidal field (see Fig. A-1). A survey of pertinent experiments indicates that the reversed-field theta pinch is the only device that has made a closed field CT without $B_{t} \cdot C^{\prime}$ 's with $B_{t}$ have been produced with a modified coaxial gun $^{4}$ and conical theta pinch guns. $5,6,7$ Most of these experiments were performed at low energy and very little is known theoretically or experimentally about CT stability and confinement, particularly in parameter regimes approaching reactor conditions.

Different names have been attached to particular types of CT's. The spheromak is conceived as a low aspect ratio tokamak with no $B_{t}$ coils and a metallic shell that is topologically spherical instead of toroidal (see Fig. A-1). It can be thought of, equally well, as a low aspect ratio RFP with $B_{t}$ going to zero at the plasma edge instead of reversing. Experimentally, increased turbulence has been observed in RFP's when the field reversal decreases to zero. This is consistent with the relatively low $B$ limits theoretically determined for spheromak stability. ${ }^{8}$ In assessing these 


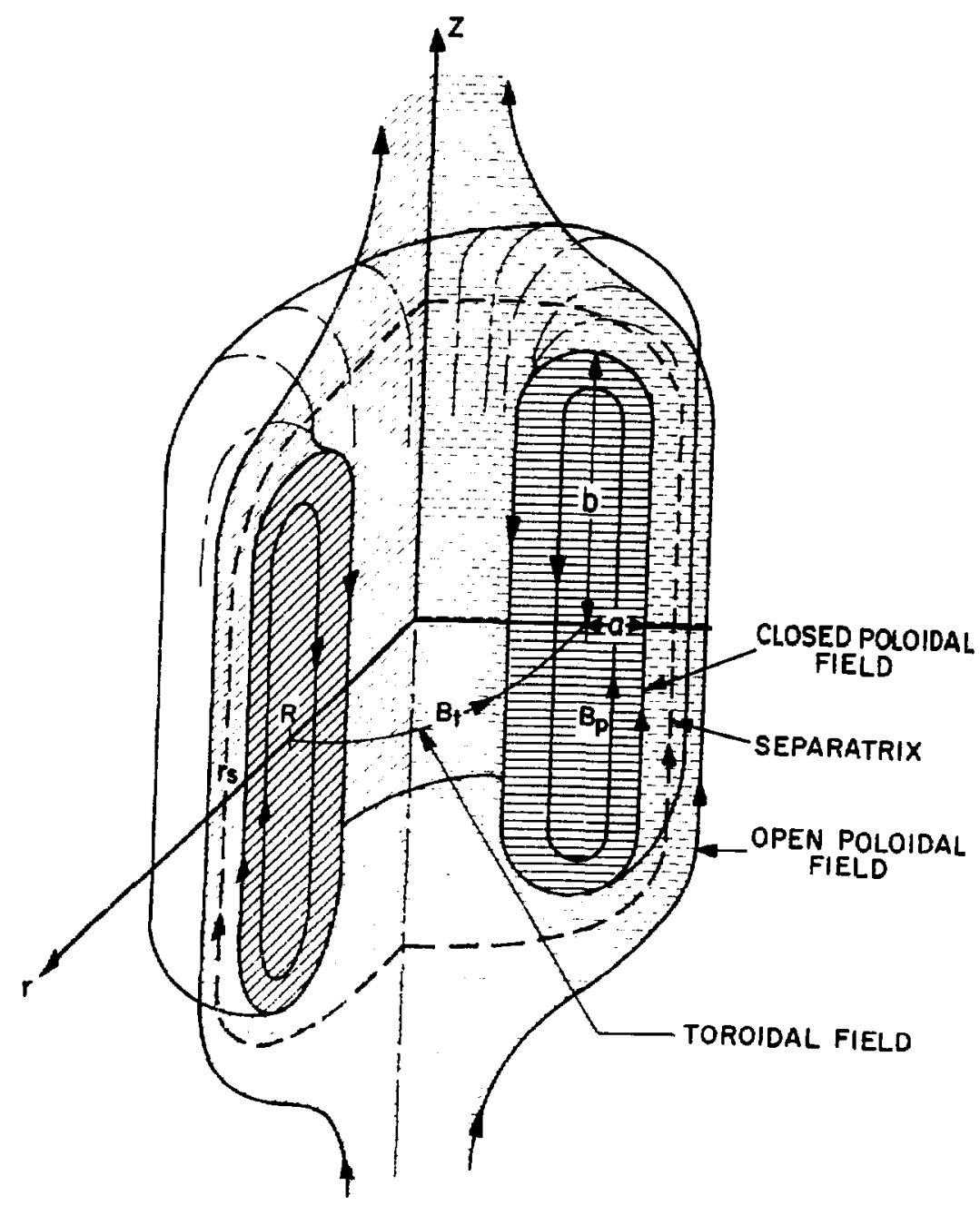

Fig. A-1.

Compact torus schematic showing the geometrical parameters $R, a$, and $b$, and the flelds $B_{p}$ and $B_{t}$. For some $C^{\prime} s, B_{t}=0$.

observations, it is useful to remember that the theoretical concept of a spheromak is the same as a CT with $B_{t}$. As mentioned above, CT's with $B_{t}$ have been produced and appear to be grossly stable. In addition, in the case of $C T$ 's without $B_{t}$, geometry effects such as large elongations (large b/a) are important to stabllity. Similar geometry effects may be important in CT's with $B_{t}$, allowing higher $B^{\prime} s$ than have been calculated. In other words, it is 
important to gain a unified understanding of all variations of CT's in order to eventually assess their reactor potential. The proposed Program Plan includes the study of CT's both with $B_{t}$ (spheromak) and without $B_{t}$.

Another variation of the CT concept is the field-reversed multiple mirror. ${ }^{9}$ As is shown in Fig. A-2, this concept consists of a series of CT's, with heir open field lines in common. The multiple mirror confinement on the open field lines allows increased pressure on the separatrix, which may be important to stability and transport in CT systems. Again, the proposed Plan includes the study of these and other physics issues culminating in a Proof-of-Principle experiment for the CT concept.

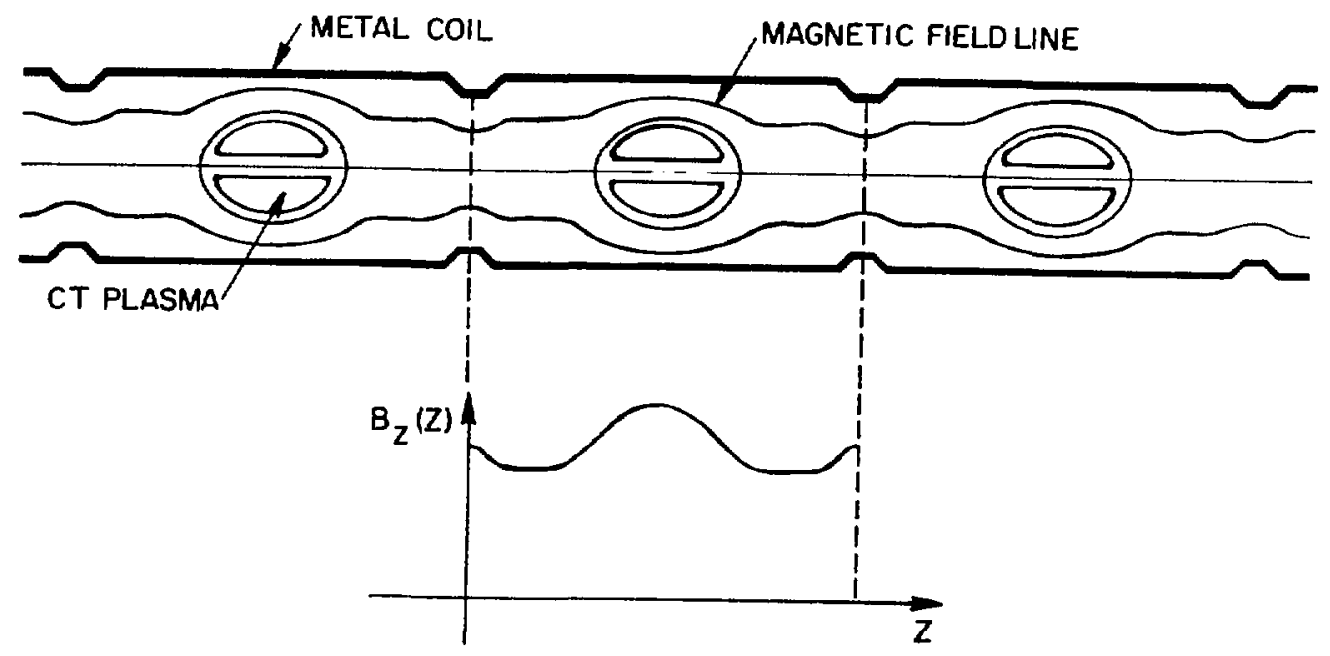

Multiple cell geometry.

Fig. A-2. 


\section{CT Reactor Systems}

The study of possible reactor systems is crucial to the LASL CT Program Plan. These studies provide both physics and technological goals which must be met for a viable reactor embodiment. Care must be taken not to rely heavily on results based on sparse and poorly understood physics data, but important information can be obtained about reactor constraints even at this early stage of research. In that spirit, three previously proposed reactor schemes are mentioned here along with some general comments about each.

The LINUS concept ${ }^{1}$ (see Fig. A-3), developed at NRL, consists of a rotating liquid metal liner which compresses a CT plasma. Some of its attractive features are that the renewable first wall also acts as a neutron $b$ lanket and breeder and supplies the adiabatic heating to ignition. The moving ring reactor (MRR) (see Fig. A-4), developed by $P G \& E^{2}$ and corne11, uses an ion-ring type of $\mathrm{CT}$, but other CT types are also usable. The ring is adiabatically compressed to ignition as it drifts into the blanket region and then continues to burn as it drifts down the tube. Like LINUS, this scheme

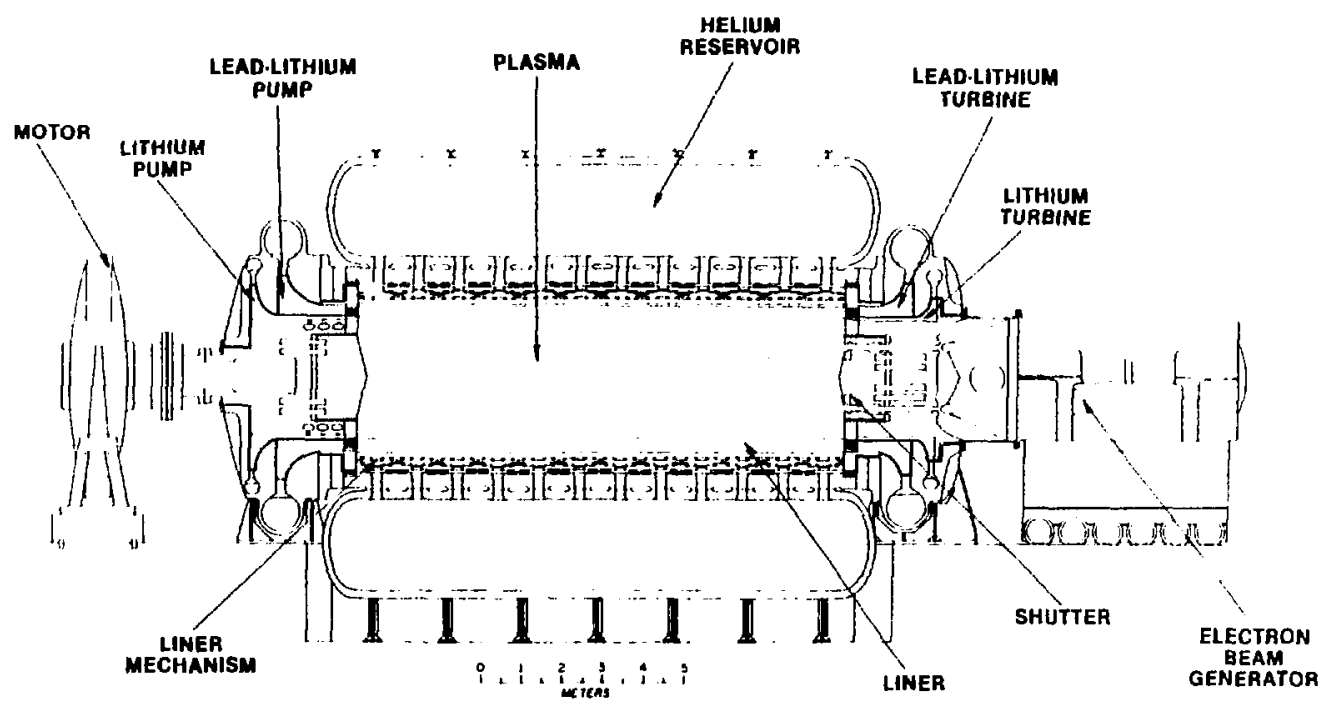




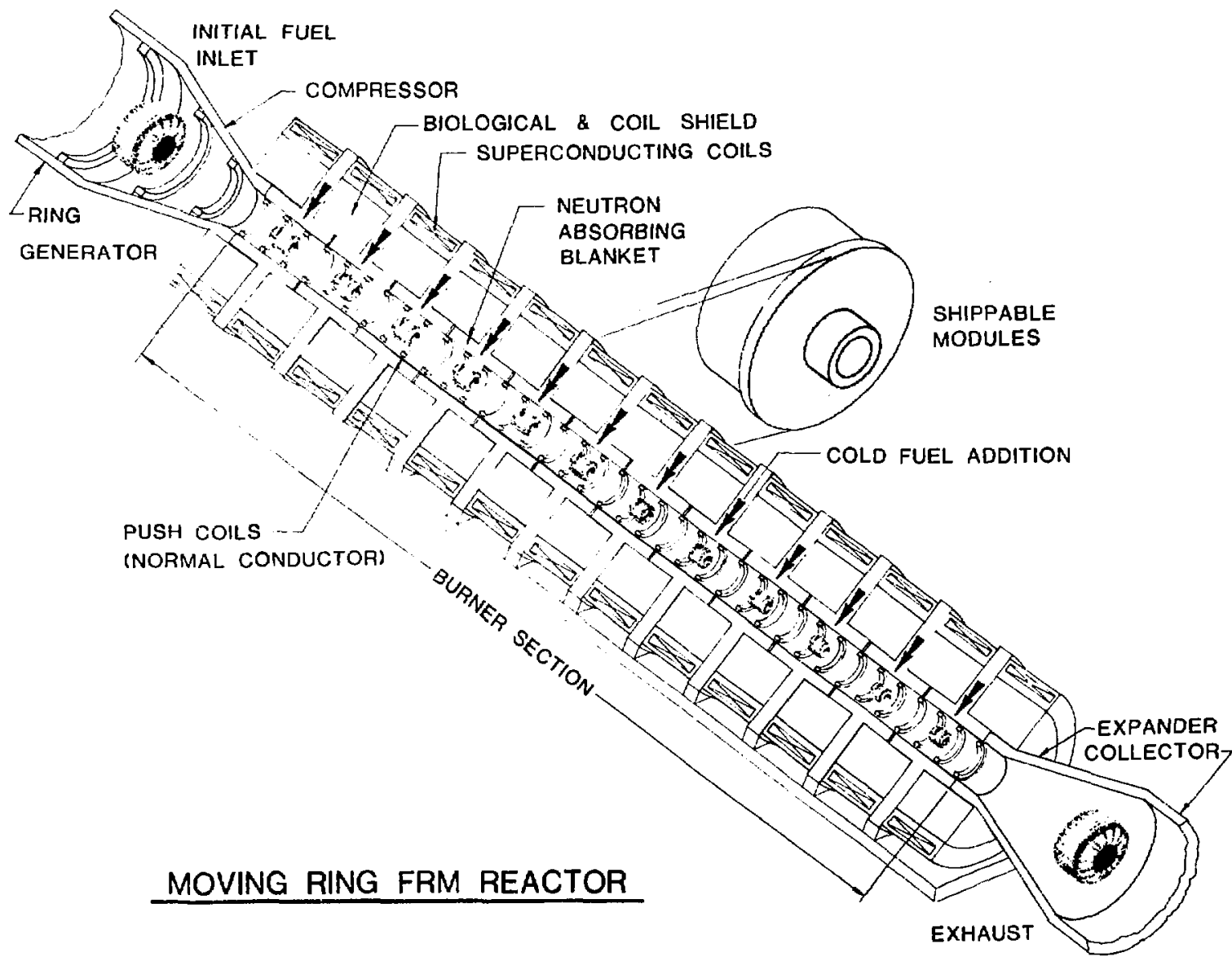

Fig. A-4.

Moving ring reactor.

uses the simple heating method of adiabatic compression, and a conducting wall can also be kept near the plasma during compression and burn. This passive liner approach avoids the mechanical complexity of cycling the liquid metal, and the first wall loading problems can be avolded by drifting the plasma instead of needing a renewable first wall as in LINUS. However, the complexity of maintaining this axial motion has been added. The field-reversed mirror ${ }^{3}$ (FRM) (see Fig. A-5), developed at LLL, uses a CT or linear array of CT's in a long-pulse or steady-state mirror configuration. The $\mathrm{CT}$ is heated and maintained with neutral beams. These beams must supply not only the heating but also must replace the particles and flux lost by diffusion for long pulse operation. The obvious advantage, however, is that the CT source does not have 
THE FIELD REVERSED MIRROR REACTOR

WOULD CONTAIN MULTIPLES OF A BASIC CELL

4

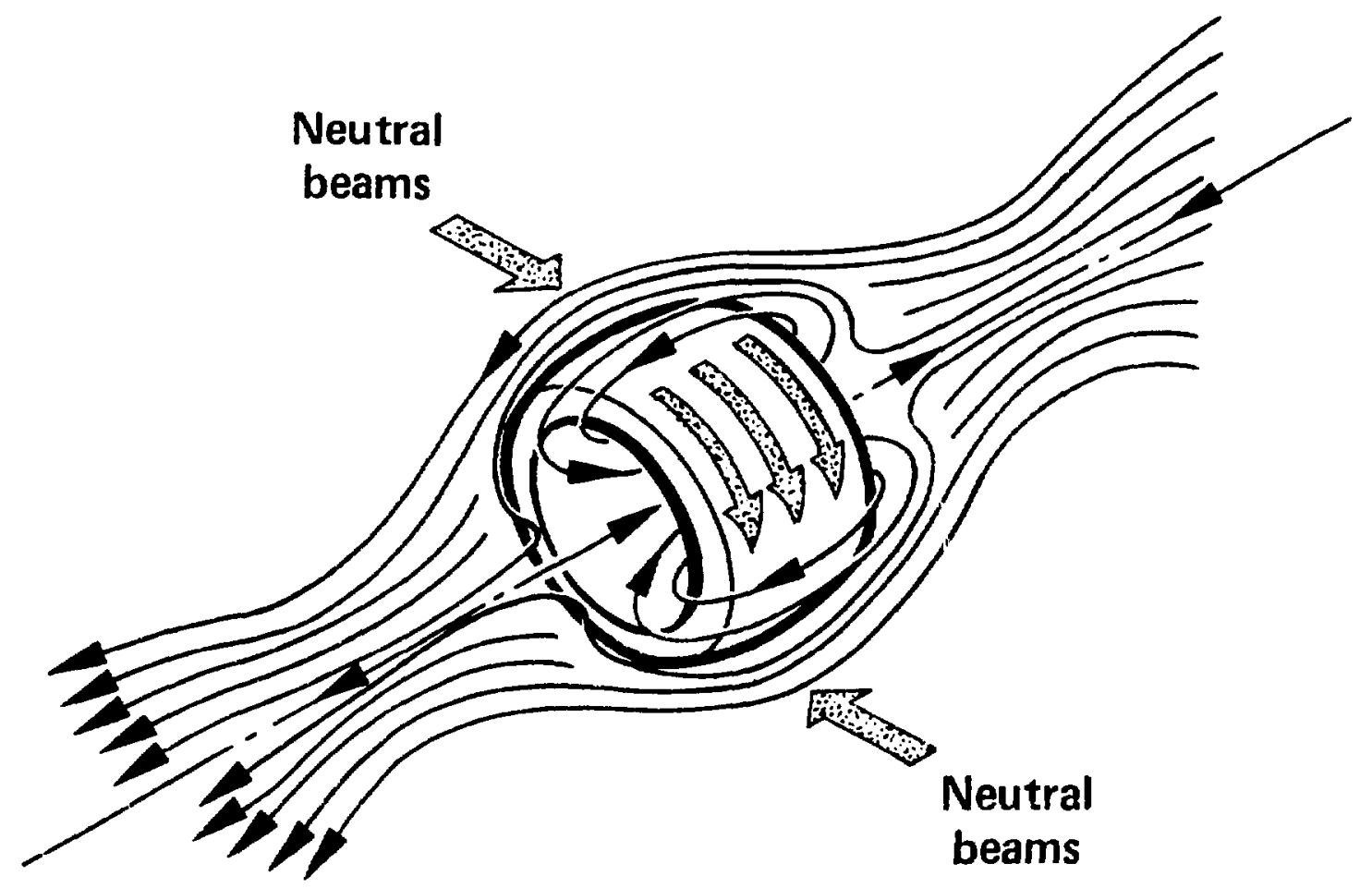

Parameter

Value

Vacuum field

$50 \mathrm{kG}$

Plasma length

$20 \mathrm{~cm}$

Plasma radius

$6 \mathrm{~cm}$

Beam energy

$\leqslant 200 \mathrm{keV}$

Fusion energy gain ( $Q$ )

5 to 9

Fusion power

$19 \mathrm{MW} / \mathrm{cell}$

Fig. A-5.

Field-reversed mirror reactor cell. 
to be operated nearly as often as in the LINUS or MRR systems. As presently conceived, all of these systems use a high-voltage, pulsed-power source for the $\mathrm{CT}$, so the size and pulse rate become a technological issue. Significant progress has been made in the recent past with this type of technology, and continued progress is needed to improve the attractiveness of all these s chemes.

The major physics issues are common to all of these reactors, but the specific impact on the design will be different. One of the major issues is the degree to which the equilibrium and gross stability depends on a conducting shell or feedback-controlled fields. Are quadrupole fields necessary or sufficient as assumed in the FRM? Is field diffusion into the metal walls slow enough so that stability is maintained during the implosion of LINUS or the drifting rings in MRR? How are these effects dependent on the plasma geometry, amount of $B_{t}$, plasma $B$, etc? Another major issue is the dependence of the transport rate of heat, particles and flux on the above mentioned equilibrium parameters. So little is known about these physics issues that an accurate assessment of the above reactor schemes is not possible at this time. However, these small, technologically attractive reactor ideas motivates the aggressive Program Plan which has been designed by LASL to answer the major physics questions. 


\section{LASL Compact Torus Physics Plan}

A. Introduction

A flow chart of the Physics Plan is shown in Fig. A-6. The production, translation, and trapping of grossly stable CT's, both with and without $B_{t}$, will be investigated experimentally. The confinement properties of both types of CT's will then be studied as single cells. The effect of multiple cells on confinement will also be studied. The most promising CT configuration will then be selected for heating studies in the single-cell form. The results of each of these studies will culminate in the design and operation of a CT Proof-of-Principle experiment. The need for a strong, parallel, theoretical program cannot be overemphasized if this proposed experimental Program Plan is to succeed. Each step of the Physics Plan is discussed below in more detail.

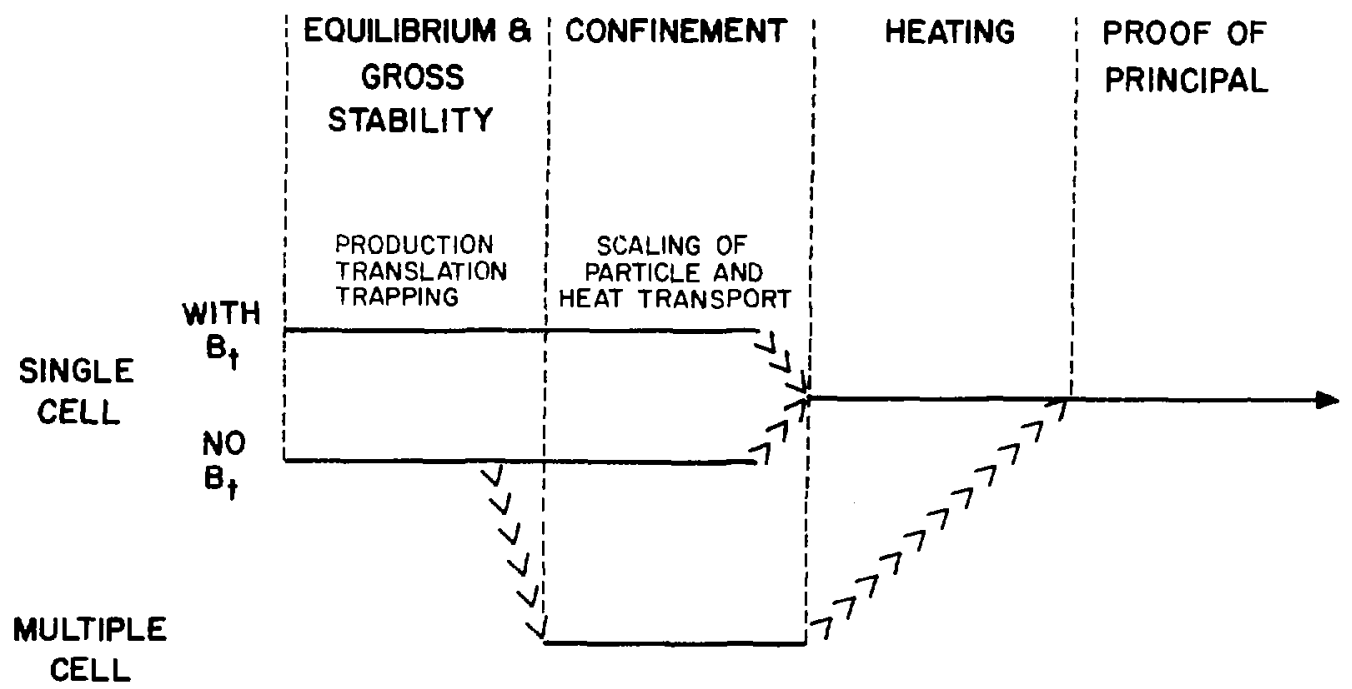

LASL Compact Torus Physics Plan.

Fig. A-6. 


\section{B. Production}

The choice of a CT source is crucial to the success of the near-term experiment as well as the reactor. Several different sources have been tried, and several more have been proposed. To our knowledge, only the three sources mentioned in Sec. I (reversed-field theta pinch, conical theta pinch, and coaxial gun) have been successful at generating $\mathrm{CT}^{\prime} \mathrm{s}$ with $\mathrm{a} / \rho_{\mathbf{i}}>1$. The philosophy of this Program Plan is to choose the sources that have the highest probability of yielding important physics results in the near term.

Since the field-reversed theta pinch (FRX system) is the only experimentally demonstrated source of CT's with no $B_{t}$, it is the natural choice for that type of CT. The formation process of the CT is shown in Fig. A-7. Once the CT is formed, it can be translated by pulsing a separately driven mirror coil (not shown) on one end of the system. This translation process has been demonstrated at Krasnaya Pachra and at LASL on FRX-A. Trapping of the CT has yet to be tried.

The conical theta-pinch gun and the modifled Marshall gun (added poloidal fleld, $B_{p}$ ) have both been used to form CT's with $B_{t}$. The Marshall gun is the preferred choice because of increased efficiency and experimental control over $B_{p}$ and $B_{t}$. The operation of the gun is shown schematically in Fig. A-8. Since this type of gun has not been operated in the high-temperature, low-density regime required in the Program, major questions still remain. Other sources such as the conical theta pinch should be considered as alternatives.

Several other alternatives that have not been successfully demonstrated have been proposed for producing CT's with $B_{t}$. The rotating hollow REB at NRL ${ }^{10}$ has formed reversed fields, but the lines intersect the wall. Passing current axially down a theta pinch (see Fig. A-9a) was proposed by LASL at the June 78 Princeton Spheromak Meeting. The use of a system similar to the old Hothouse-I facility at UCB is being considered at the University of Maryland (see Fig. A-9b). Several other techniques have also been proposed by PPPL. After considering each of these schemes, it appears that the FRX system and the modified gun have the best chance of producing CT plasmoids that can be translated and trapped for detalled physics study in the near term. 

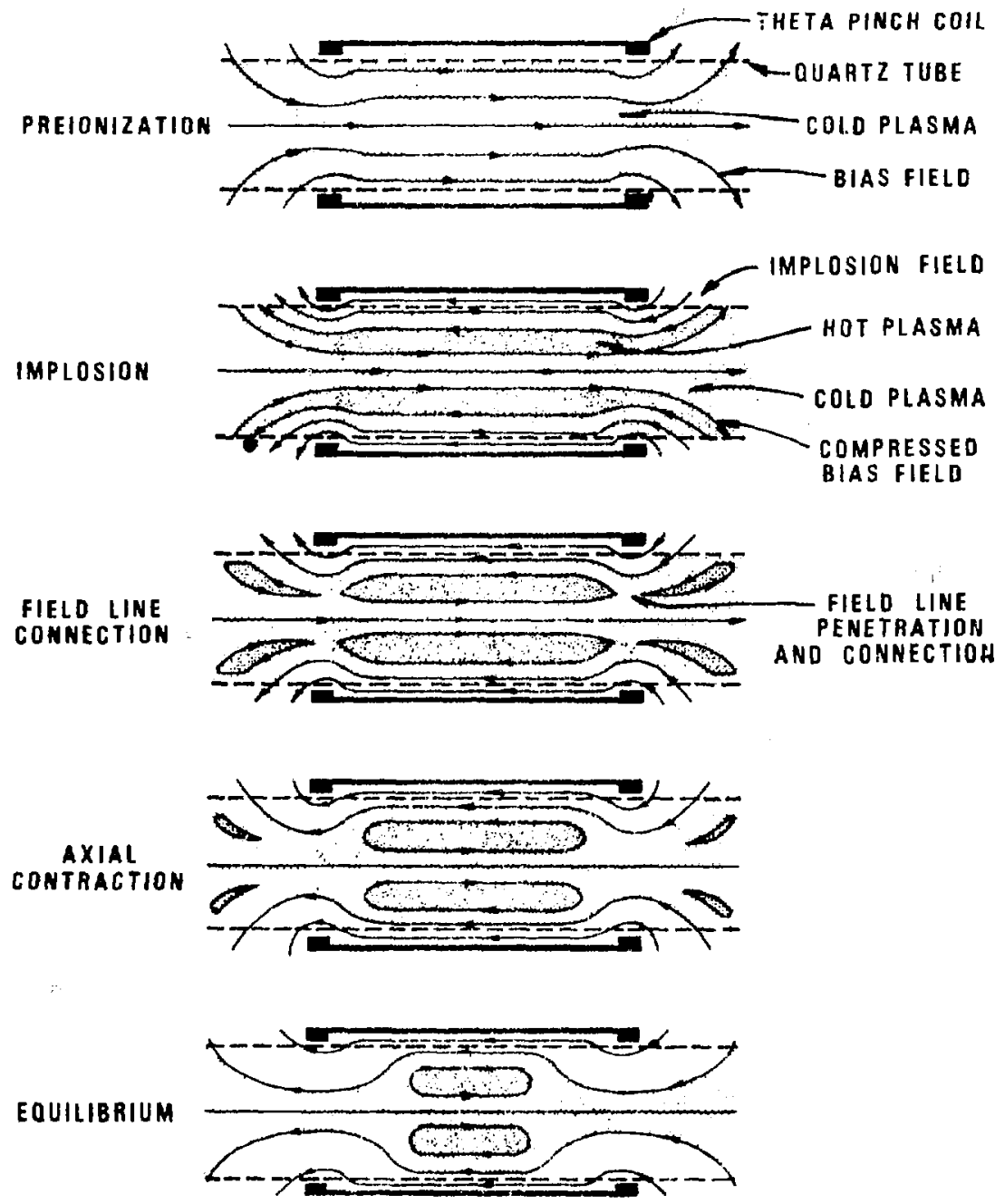

Fig. $A-7$.

Formation sequence of a CT in a field-reversed theta pinch.

\section{Equilibrium and Gross Stability}

Once the plasma has been trapficd, the dependence of gross stability on a variety of equilibrium parameters cin be studied. The gross shape of the CT, such as the values of $\mathrm{k} / \mathrm{a}$ and $\mathrm{b} / \mathrm{a}$, appear to be important. Metal walls may be required to maintain the optimum shape of the equilibrium or they may be needed to wall stabllize gross MHI) modes. The need for metal walls may be relaxed by using quadrapole fields. These issues need to be addressed experinentally and theoretically as well as the ifects of pressure profiles, pressure on the separatrix, ratios of $\mathrm{B}_{\mathrm{t}} / \mathrm{H}_{\mathrm{p}}$, and $\beta$ an the gross stability of the plasma. 


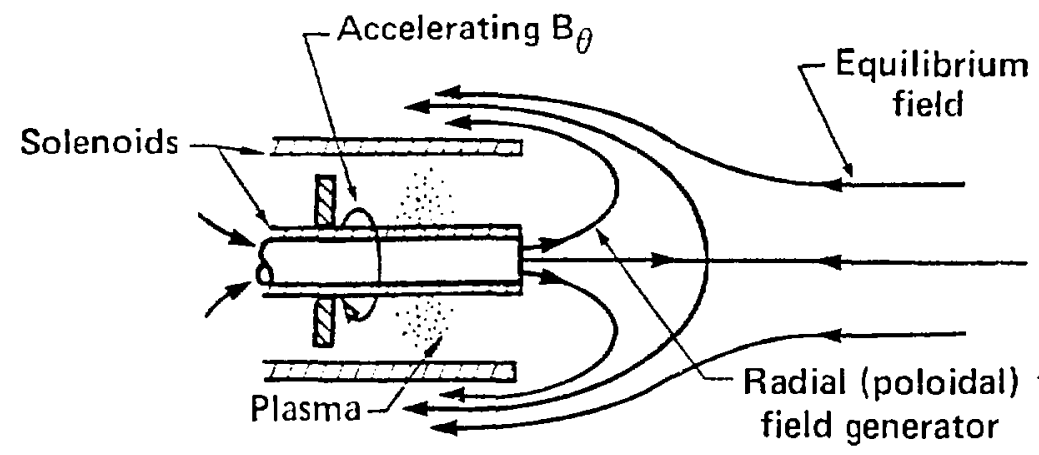

(a) During acceleration

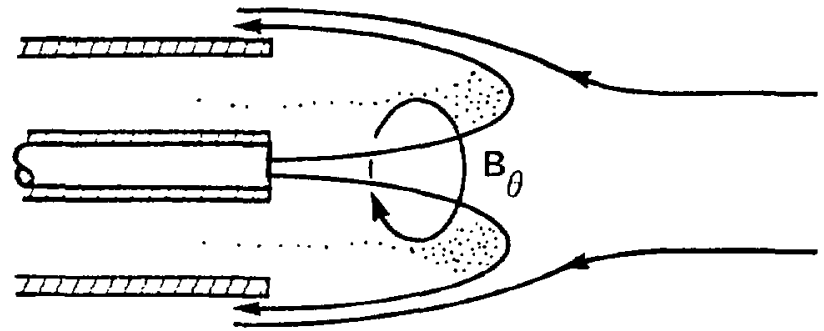

(b) Later

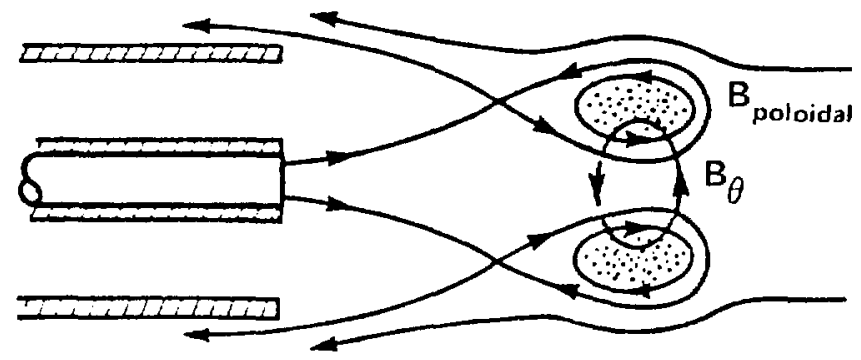

(c) Still later Fig. A-8.

Gun production of a CT (Lawrence Livermore Laboratory).

D. Confinement

The confinement properties of CT's are not likely to be classical. Internal, higher order MHD modes, resistive modes, and microturbulence from a varlety of sources are expected to enhance cross-field transport. The important parameters that need to be varied in the experiment include all of the equilibrium parameters mentioned above plus shear, $a / \rho_{1}\left(\rho_{1}\right.$ is the 1on gyroradius) and $\omega_{c 1} \tau_{1}$ ( $\omega_{c 1}$ is the ton gyrofrequency and $\tau_{1}$ the ion collision time). In particular, theory indicates that an optimum value of a/p may exist. This optimum arises from the opposite a/ $\rho_{1}$ scaling of two possible sources of transport. Increased $a / \rho_{1}$ decreases transport from modes such as 


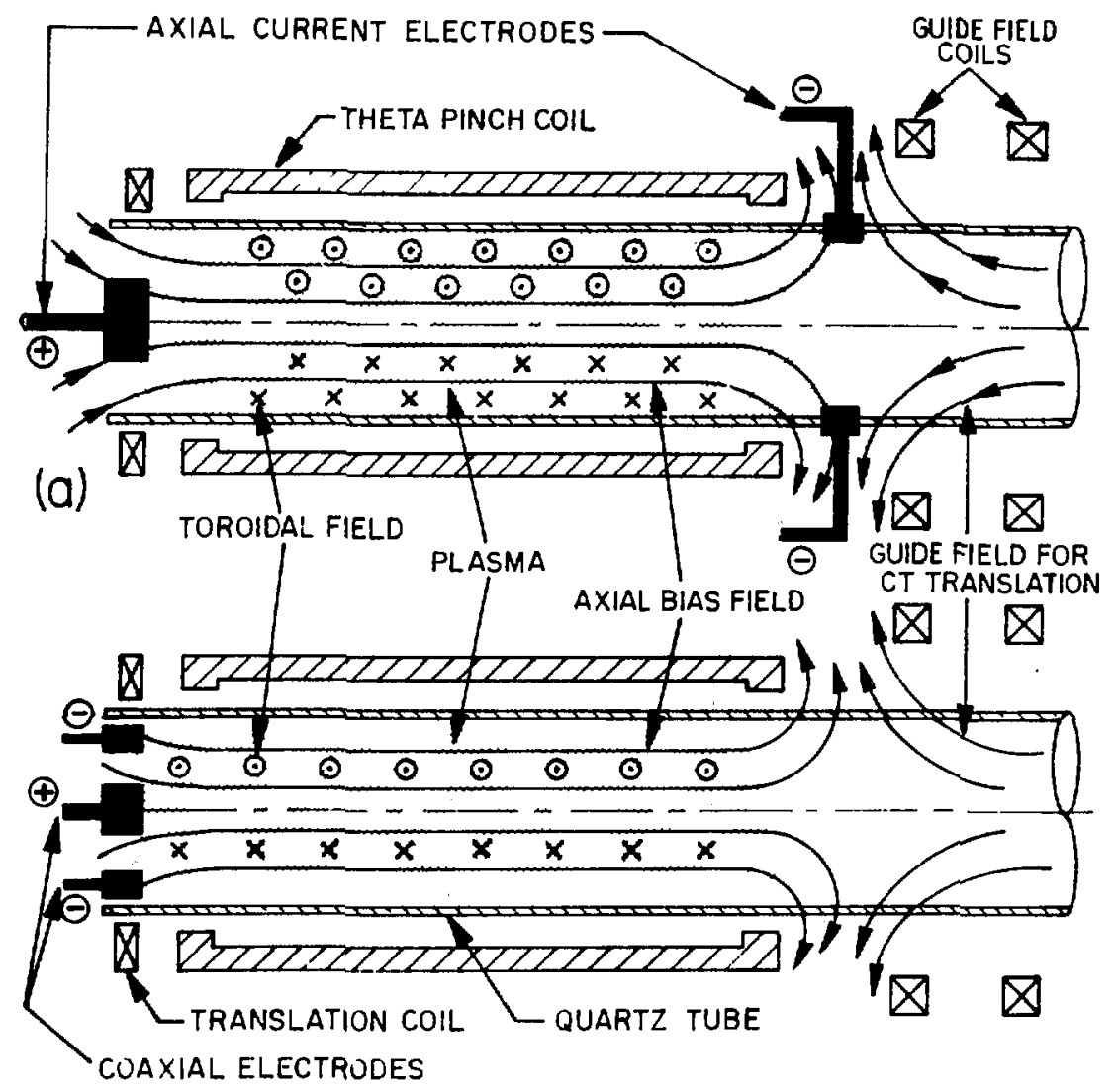

(b)

Fig. A-9.

Formation of a CT with $B_{t}$ using two modified theta-pinch geometries. The two geometries are shown just prior to the implosion with fields antiparallel to the Indicated axial blas field. The subsequent reconnection of the antiparallel fields near the ends should be similar to the sequence in Fig. 7 . (a) An axial current is drawn between the electrodes to establish $B_{t}$ just prior to implosion. (b) An ionizing shock propagates axially from the coaxial electrodes to establish $B_{t}$ just prior to implosion.

the lower hybrid drift, but decreased $a / \rho_{i}$ provides enhanced FLR stabilization of MHD modes. Preliminary experimental results indicate that increased lifetime results from increased $a / \rho_{i}$. Future experiments must extend this study of transport to larger values of $a / \rho_{i}$ and to higher temperatures. In 
addition to the single-cell studies, experiments are needed to test the enhanced confinement predicted for the multiple-cell geometry.

E. Heating

The results of the confinement studies will be used to select the CT equilibrium parameters which are most promising for the heating studies which follow. Three types of heating schemes appear the most attractive at the present time: 1) neutral beams as proposed for the FRM, 2) adiabatic compression as proposed for the MRR and LINUS concepts, and 3) RF heating, most likely in the form of magnetosonic heating. These heating approaches need more careful examination before one or more is chosen for the heating experiments. Care must also be taken that the heating scheme does not enhance the transport unacceptably or drive the CT grossly unstable. If the heating, transport, and equilibrium studies are compatible with a reactor, then a Proof-of-Principle experiment becomes the logical next step. 
IV. Description of LASL Compact Torus Experimental Plan

A. Introduction

In Fig. A-10 the plan is shown for a series of experiments to accomplish the physics objectives discussed above. The constraints assumed in cost and manpower are explained in Sec. $V$ below. Each box represents the interval of an experiment's operation with arrows showing the flow of needed information for

\section{LASL COMPACT TORUS EXPERIMENTAL PLAN}
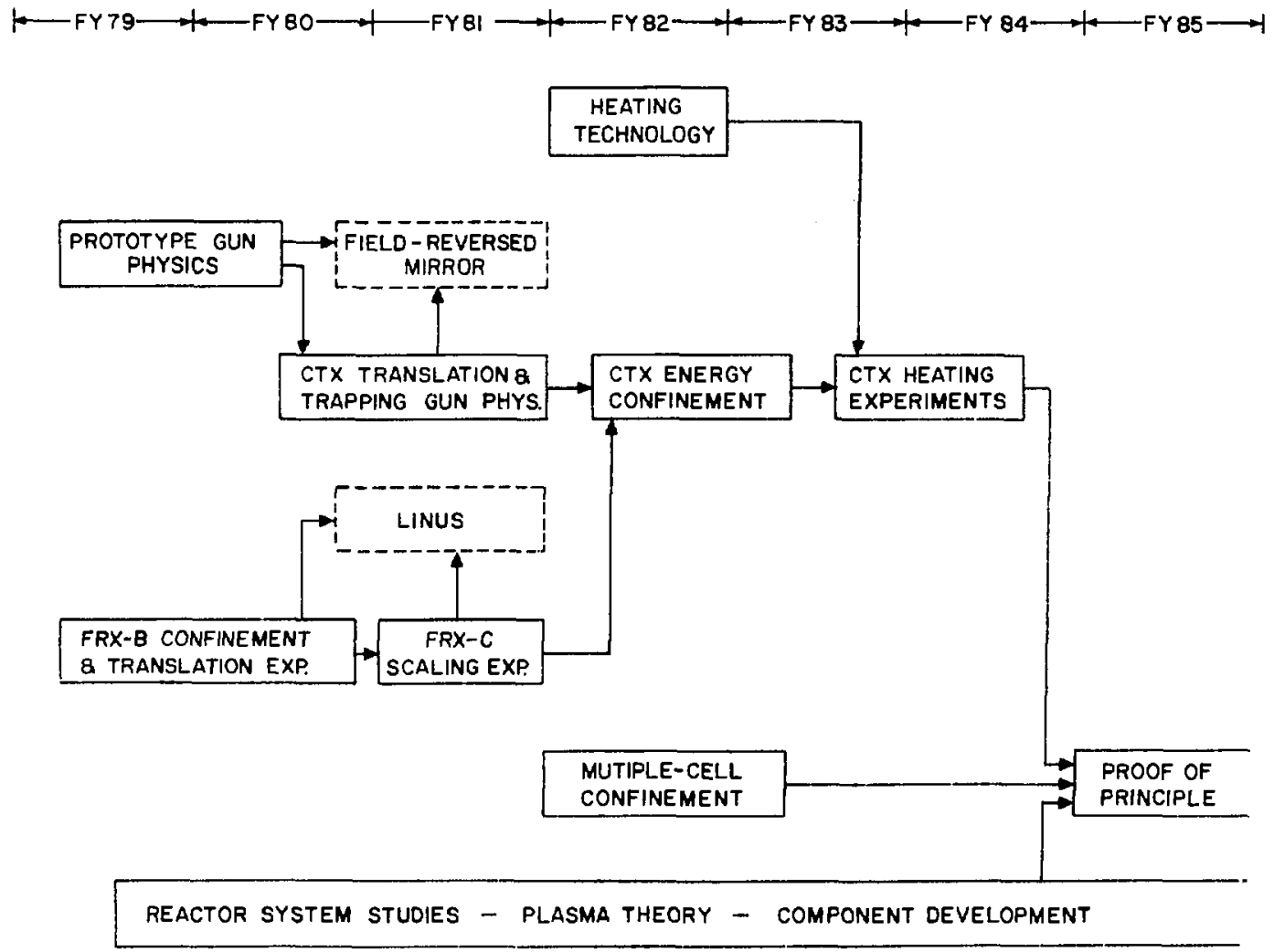

Compact Torus Experimental Plan.

Fig. A-10. 
other subsequent, or in some cases, concurrent experiments. Fiscal years indicated at the top show the approximate time scale.

In addition to the specific experiments indicated in Fig. A-10, other CTR-Division activities are important to the success of this program. The most important are the ongoing reactor studies indicated as a long bar at the bottom of Fig. A-10. The interaction between LASL's system studies and experimental groups hopes to ensure that all experiments are designed to test the relevant physics and technology, but for simplicity a single arrow is shown contributing to the Proof-of-Principle experiment. Similarly an ongoing theory program and technology development program contribute at all phases of this experimental plan. Component development in the areas of pulsed power, RF heating, and neutral beam injection is anticipated to be needed in this program.

Computer control and data acquisition methods already developed in previous LASL experiments are adequate for the near term plans. Clearly future larger experiments will require engineering and development in this area. Diagnostic measurements, an essential part of all plasma experimental physics, are reasonably well developed and are constantly being refined at LASL for this plasma regime although new developments will be needed when the program reaches the phase of heating studies. The measurement of internal magnetic fields (without perturbing the plasma) is a difficult diagnostic of particular Importance in CT configurations with their intrinsically complex field patterns. Fortunately this need also exists in the RFP program, and concerted efforts are underway in the LASL CTR-Division to develop methods of measuring internal magnetic fields.

The research effort represented by this Compact Torus Experimental Plan both compliments and benefits from the main CTR-Division program to develop the RFP concept. Although the experimental methods of plasma production are rather different, both programs study the physics of an axisymmetric torus in approximately the same regimes of density and temperature. Diagnostic techniques are of mutual interest as already mentioned with regard to magnetic field measurement. Another example is the development of space-resolved Thomson scattering for ZT-40 which can be applied to the CT program. Concepts and theoretical studies developed in one program are likely to benefit the other. For example, the elongated prolate geometry of the field-reversed theta pinch has proven experimentally to be beneficial for plasma stabilty. As theoretical understanding of this result is obtained, it becomes possible to 
investigate whether a similar geometrical modification would improve the RFP configuration. Other CTR-Division activities also compliment the CT program. Precise heat flow measurements in the Q-machine are important for understanding the physics of plasma outside the separatrix of CT's. The multiple-cell experiment described in IV-E will particularly benefit from such studies.

\section{B. The FRX-B Experiment}

The development of CT's without a toroidal magnetic field begins with the presently operating FRX-B experiment (Fig. A-11). As shown in Fig. A-10, the experiment operating through FY 1980 contributes the main.data base for a future scaling experiment, FRX-C, and a multiple-cell confinement experiment. To date the FRX-B has established that a CT with a grossly stable equilibrium can be formed. The diagnostic measurements underway will establish a data base with a limited range in $a / \rho_{i}$ and geometrical parameters provided by varying the initial filling pressure, initial bias, and so forth.

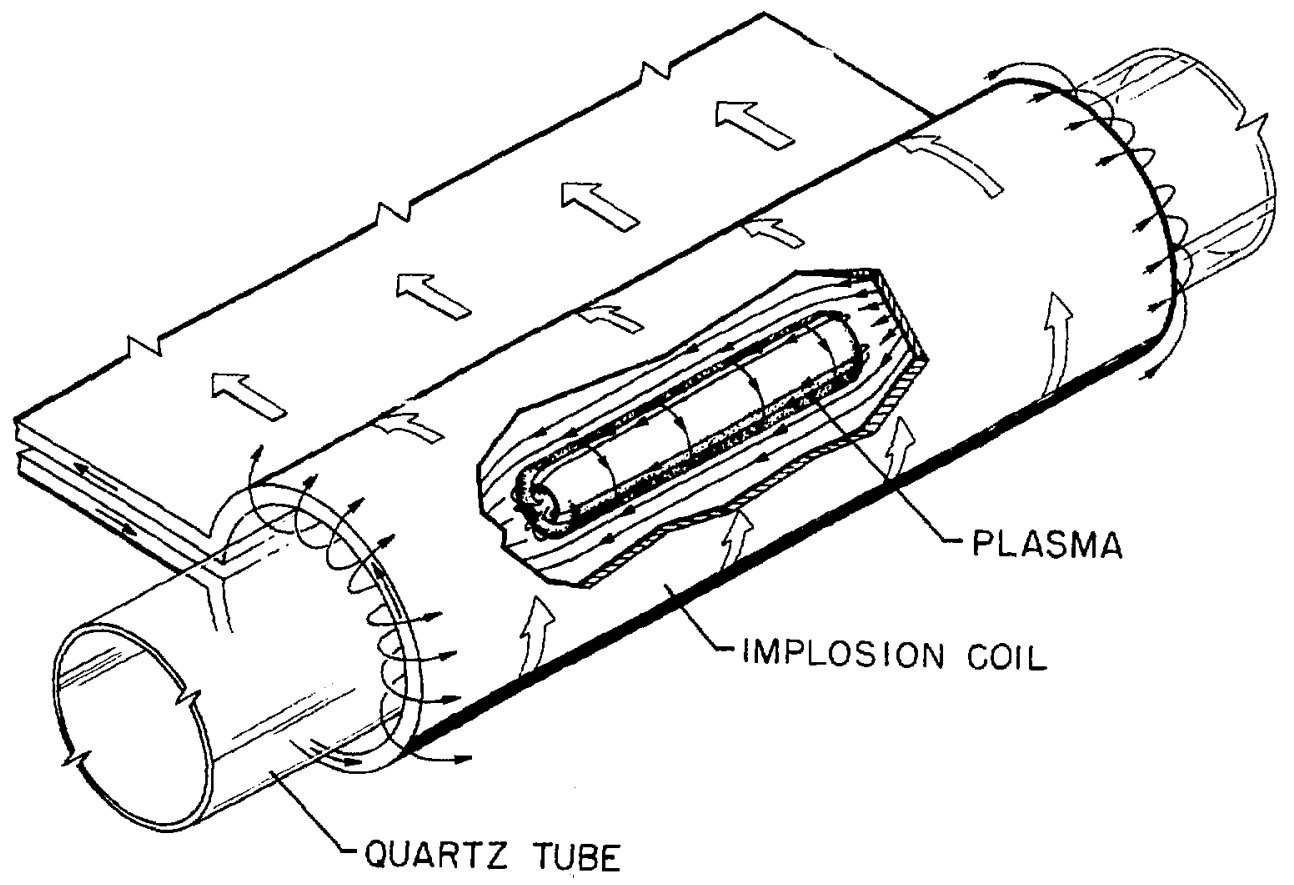

FRX-B coil and plasma geometry.

Fig. A-11. 
One important physics issue addressed by the experiment is the origin of the plasma rotation which leads to an $m=2$ disruption. At present it is believed that the rotation results from plasma particle losses, but more complete diagnostics are required to establish this interpretation experimentally. If the present interpretation is correct, the $n=2$ mode develops after more than one-half of the plasma is lost. Therefore, the mode is not the primary cause of plasma loss. Thus the plans to explore scaling aimed at better confinement will also address the $m=2$ rotational mode.

Before termination of FRX-B near the end of FY 1980, it will also be possible to make initial tests of translation and trapping in an external quasi-steady coil. The development of pulsed gas techniques and pre-ionization methods underway for FRX-B translation experiments will be of general applicability in the CT program. Translation experiments will provide new physics information about CT's. For example, the need for a netal wall in close proximity to the plasma can be investigated because the geometry of metal walls can be varied in the guide-field region.

It should be noted that results from the FRX-B experiment contribute in an important way to the LINUS program. Based on data obtained in FRX-B and the scaling experiment described below, it should be possible to establish whether theta-pinch generation of a CT is adequate for the requirements of LINUS.

\section{The FRX-C Experiment}

The FRX-C is a scaling experiment which extrapolates the FRX-B results towards the higher temperature and longer lifetime needed for fusion. Scheduled to begin operation late in FY 1980, as shown in Fig. A-10, it provides a means for production and injection of plasma with no toroidal field into a steady mirror field.

Based on field-reversed theta-pinch experiments at LASL and elsewhere, it can be shown that collisionless high-temperature CT's produced by theta-pinch methods require large applied voltage with minimum subsequent compression before translation. The hardware needed for the experiment shown in Fig. A-12 is largely available from parts salvaged from the Staged Theta Pinch Experiment. Technology developed in that experiment and the Implosion Heating Experiment lead to confidence that a high energy $C T$ can be produced with temperature in the $\mathrm{keV}$, range. Compared to FRX-B a larger coil diameter $(45 \mathrm{~cm})$ 


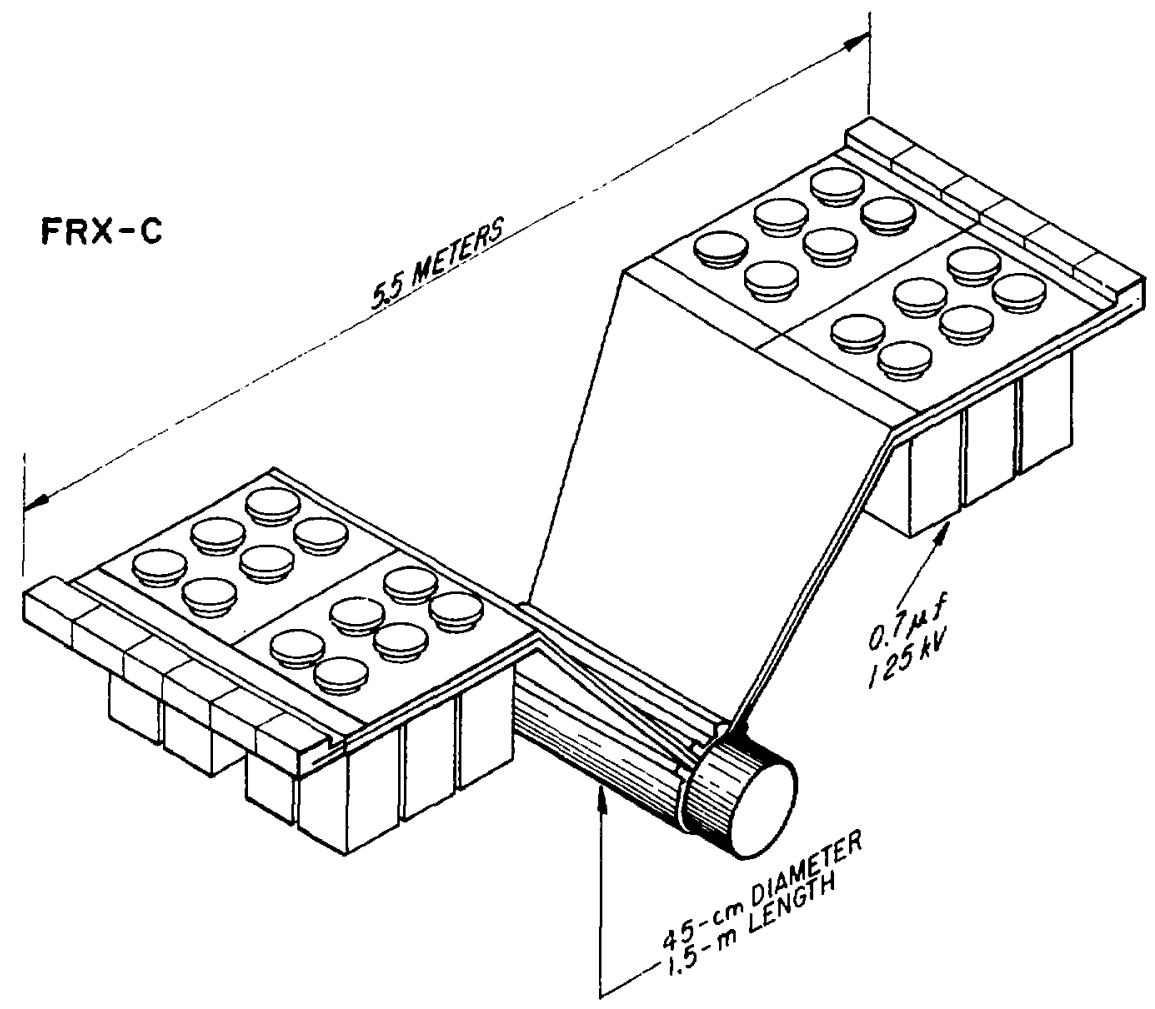

Fig. A-12.

FRX $-C$ main bank, collector plate and coil geometry.

and length $(150 \mathrm{~cm})$ combined with higher temperature provide key tests of the scaling of CT's with geometry and $a / p_{i}$.

The configuration shown in Fig. A-12 is chosen to permit locating the FRX $-C$ experiment adjacent to the CTX vacuum vessel and mirror coil described below. This allows translation and trapping of the theta-pinch produced CT into a steady-state magnetic mirror where the confinement can be measured. Plasma is transferred through a quasi-steady solenoid designed to decouple the high-voltage generator from the mirror colls.

\section{CTX Prototype Gun Investigations}

The first plasma source for the CTX facility, to be described below, will be a coaxial plasma gun with pololdal field components. This possible method of producing CT's having both toroldal and poloidal fields was described in part I. The scheme follows the recent suggestion of $C$. W. Hartmanll and the 
much earlier experiments of Alfvén and coworkers 4 with low energy guns containing the required poloidal field (see Fig. A-8). Typically, the high energy guns without poloidal field tested at LASL $^{12}$ have produced two velocity components. The fast component has the right range of energy and particle inventory needed for the CT and FRM programs, but, unfortunately, the slow component contains about 100 times the desired inventory. The key experimental issues are whether the slow component can be eliminated or rendered harmless and whether the fast component can be trapped in the desired closed-field configuration.

The use of plasma guns as a source for producing CT's is also being investigated at LLL in their Field Reversed Mirror (FRM) program. The present effort at LASL was started as a cooperation with LLL because the numerous issues and questions to be investigated require both programs to get answers on a timely basis. The Livermore approach employs an only slightly modified LASL gun operating in the usual density regime. An important question is whether this approach can induce the necessary magnetic field line reconnection to separate the fast and slow components. The LASL approach involves more extensive modification to the gun in an attempt to operate at lower densities and eliminate the slower component. There are indications from the work of Marshall and Henins in 1964-65, 13 that the addition of a bias field (i.e., a magnetic field in the gun barrel parallel to the gun electrodes), as is present in our new gun, may indeed allow operation at somewhat lower than conventional densities. They also found that the presence of a bias field supressed the formation of the slow plasma component.

Figure A-13 shows a schematic diagram of the new gun being constructed at LASL. The radial dimensions of the gun have been altered to achieve a ratio between the inner and outer electrode diameters nearer to unity than is normal for LASL coaxial guns. This may decrease the tendency, believed to originate from the $1 / \mathrm{r}$ dependence of the driving magnetic field, for the gun to produce fast and slow components of the output plasma. A pair of magnetic field coils, called the poloidal field coils, have been introduced just inside and outside of the inner and outer electrodes respectively. These coils are run in series and produce the largely radial field at the muzzle of the gun that forms the poloidal.field for the CT. The bias field coil is placed outside of the outer poloidal field coil. In order to provide easy access to the inner poloidal field coll and to allow diagnostic access down the axis, the usual arrangement 


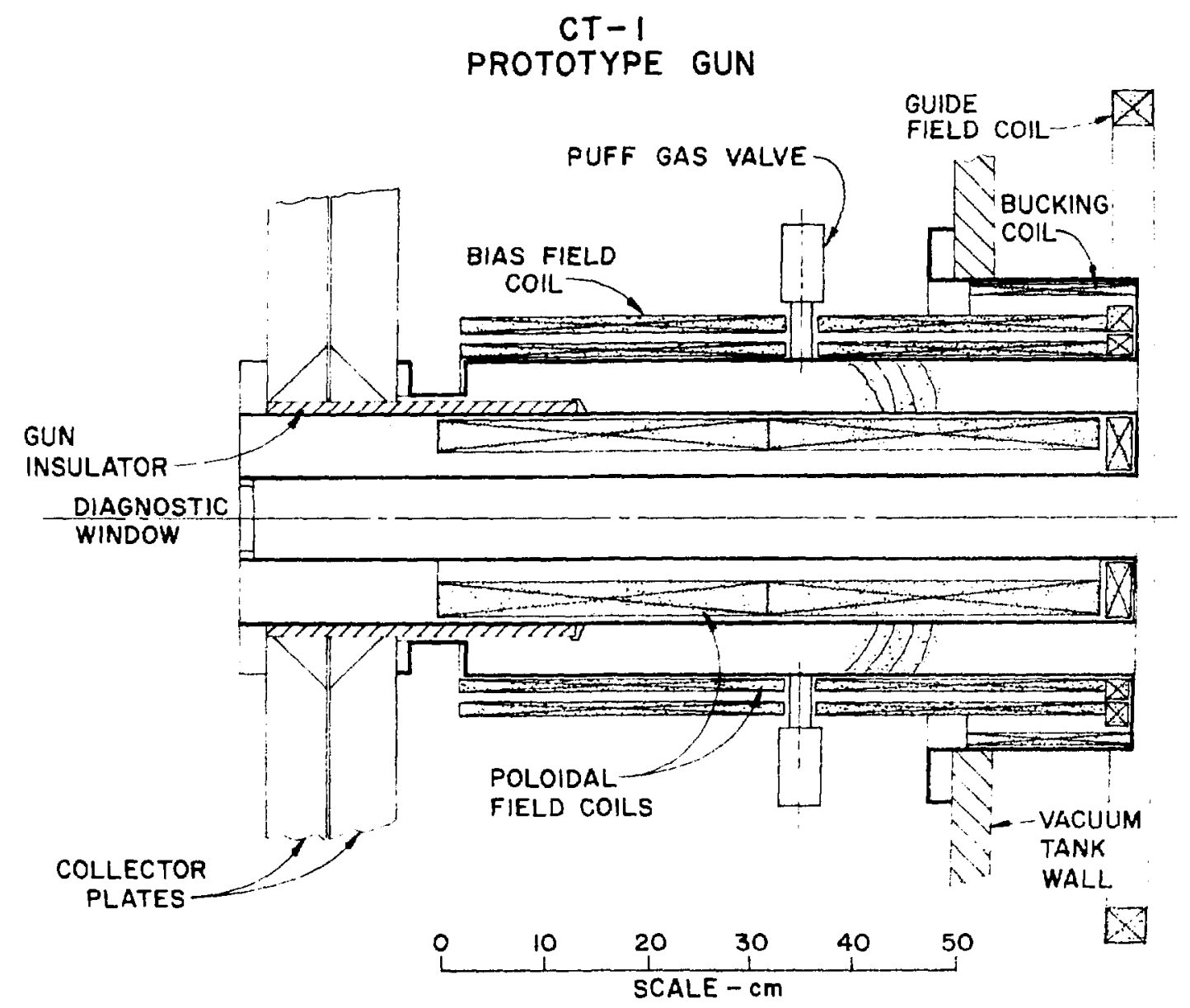

Fig. A-13.

Cross section of modifled LASL gun.

of a single gas puff valve on axis has been changed to having six $s$ izultaneously operated puff valves feeding gas through the outer electrode. is shown in Fig. A-10, the initial experiments will be done using the -ojfíiec plasma gun on the present gun facility. This work will be done in EExa:LE: with the construction of the CTX facility. The emphasis of these in:estizations will be on the problem of the production of $C T^{\prime} s$ with toroidal fielis. Otiner issues such as translation and trapping of the tori, energy 
containment time, etc., will be investigated later on the CTX facility itself. It is anticipated that the effect of the variation of a variety of parameters (such as the gun voltage, the strength of the bias and poloidal magnetic fields and the amount and timing of the neutral gas puffed into the gun) will have to be investigated before it is learned how to produce CT's from a gun source.

\section{E. CTX}

CTX provides the central facility for LASL's Compact Torus program. It is presently being installed in the area formerly occupied by the Staged Theta Pinch experiment. Figure A-14 is a side view of CTX. The central element of the facility is a 4.6-m long, 1.5-m diameter vacuum tank fitted with external DC magnetic field coils. Fortunately, the coil/tank system already exists at LASL, having been obtained years ago for a different experiment. A new power supply for the DC coils will provide a guide field of up to $10 \mathrm{kG}$, so the system is ideal for studying the trapping and the confinement properties of CT's.

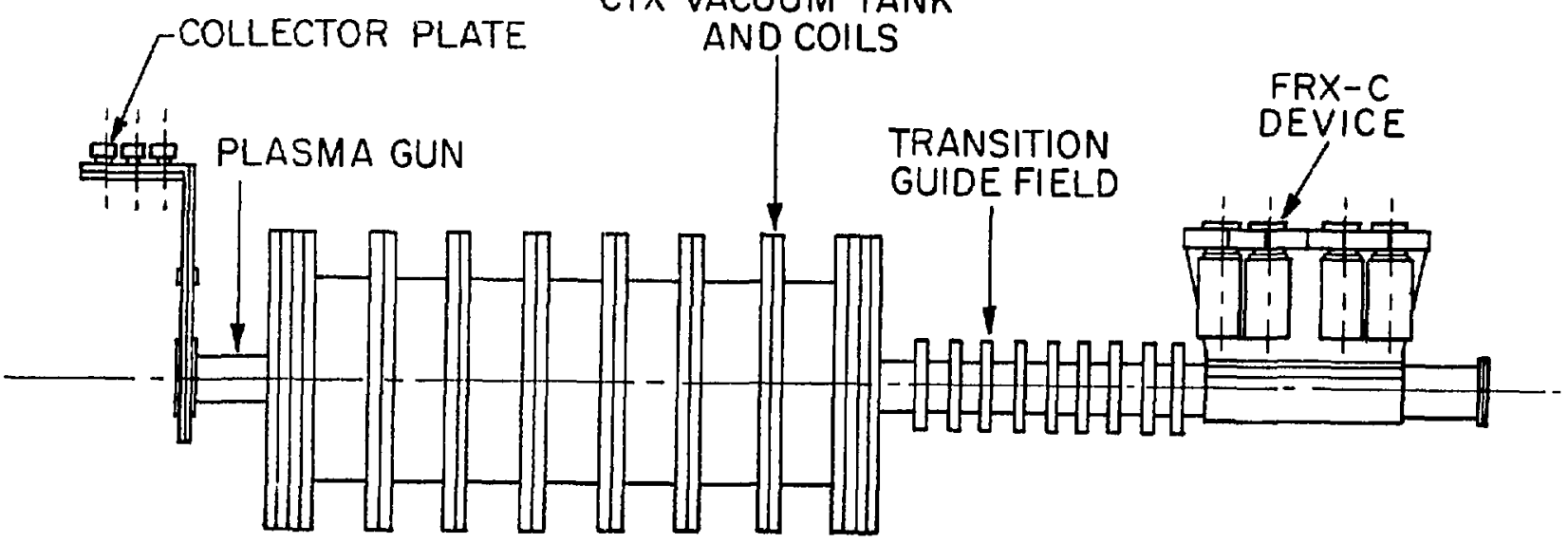

Side view of CTX showing the gun and FRX-C sources. 
Both ends of the CTX central vacuum tank are available for plasma sources. Compact tor from two sources, then, can be studied on the same facility with common diagnostics. If the schedule demanded it, and if the necessary funding were available, one can imagine operating the two sources "simultaneously" by dual shift operation of the facility. As is shown in Figs. 14 and 15, it is presently planned to install a plasma gun source in one end and the FRX-C source in the other end, thus allowing the study of CT's with and without toroidal fields. As is shown in Fig. A-10, the first experiments on CTX will address the equilibrium and gross stability issues of gun-produced CT's. In particular, the problems of translation, trapping, the necessity of nearby metal walls, etc., can be studied, the questions of production having already been investigated in the prototype gun experiments. Later, when the properties of the FRX-C plasmas have been established, this source will be connected to CTX. With the two different sources the effect on the energy containment time

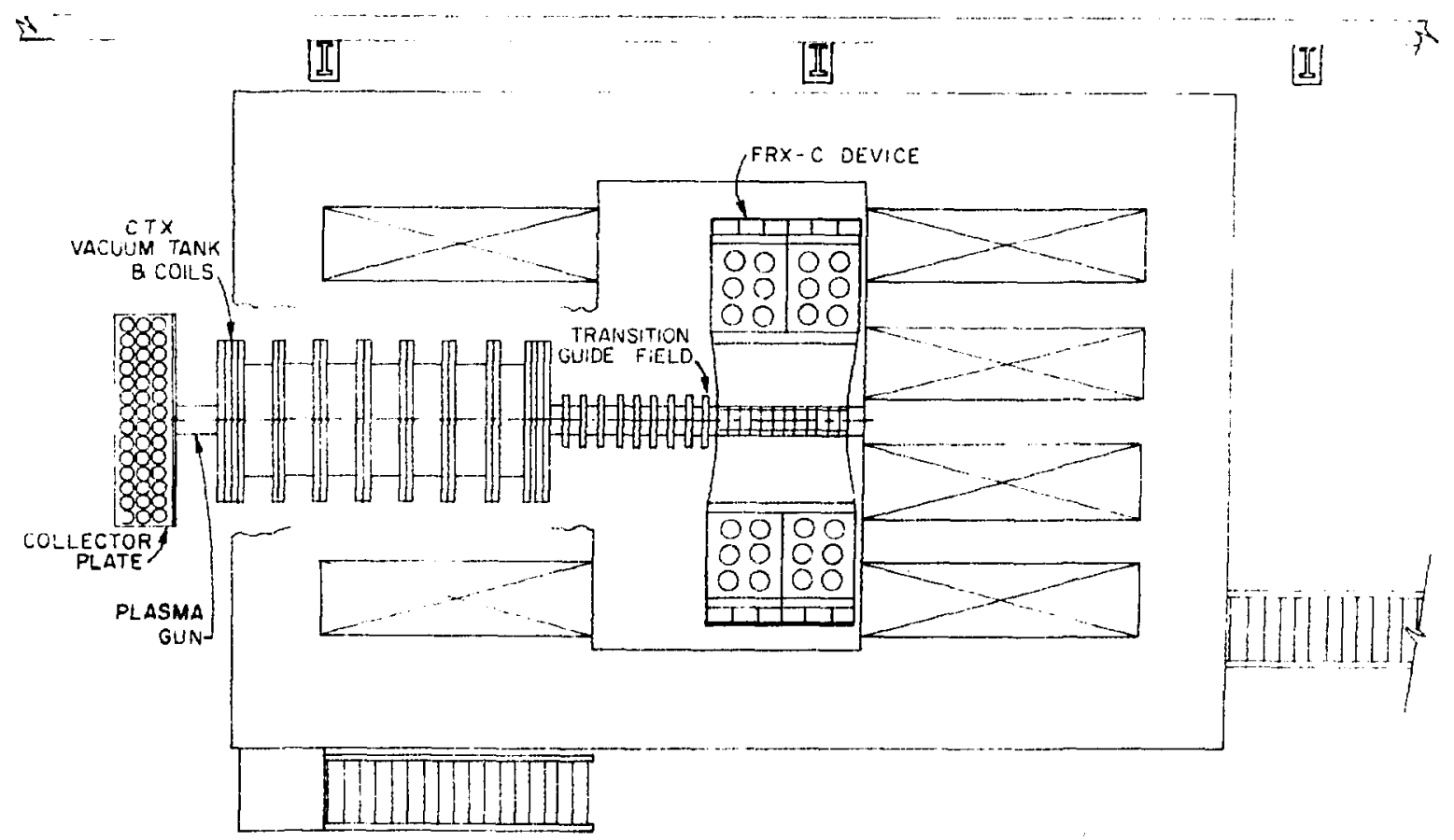

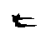
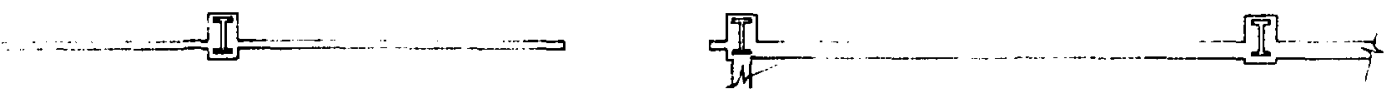

Fig. A-15.

Plan view of CTX in the experimental area. 
of such parameters as magnetic field shear, $b$, the geometry of the torus, $a / p_{i}$, etc., can be studied. These investigations concerning energy and particle confinement in $\mathrm{CT}^{\prime} \mathrm{s}$ will occupy more than a year and will address the single-cell confinement section of the Physics Plan.

During the period of the confinement studies it will be necessary to study various heating options for the next phase of the Physics Plan. Possible options are neutral beam heating, RF heating, (magnetosonic and other possibilities), and compressional heating. More than one type of heater needs to be developed because it is possible that a particular heating method could cause equilibrium stability or transport problems for the type of CT chosen for heating studies. Therefore, we anticipate a parallel effort to develop components and hardware for the heating options. During this period it will also be necessary to obtain familiarity with new (to LASL) diagnostics such as charge exchange techniques.

E. Multiple-Cel1 Experiment

Theory indicates that confinement of a CT is related to the plasma pressure profile, particularly the pressure on and near the magnetic field separatrix. A significant increase of confinement for plasma lost on open field lines is possible by using the effect of multiple magnetic mirrors in a linear array of CT's. There is a clear need to study the physics of such a configuration before deciding on the optimum reactor embodiment and the associated proof-of-Principle experiment. Thus the Compact Torus Experimental Plan, Pig. A-lO, includes conversion of the present Scylla IV-P device into a multiplewcell experiment in FY 1981 with operation in FY 1982. The arrangement shown in Fig. A-16 assumes that Scylla IV-P is modified to produce plasma with the same parameters as $F K X-B$ in all respects except length and number of plasma cells. A data base therefore exists to compare single-cell and multiple-cell confinement. Variations in cell length determined by the periodicity of the external. coil permit study of the resistive tearing mode and confinement for a variable number of cells. 


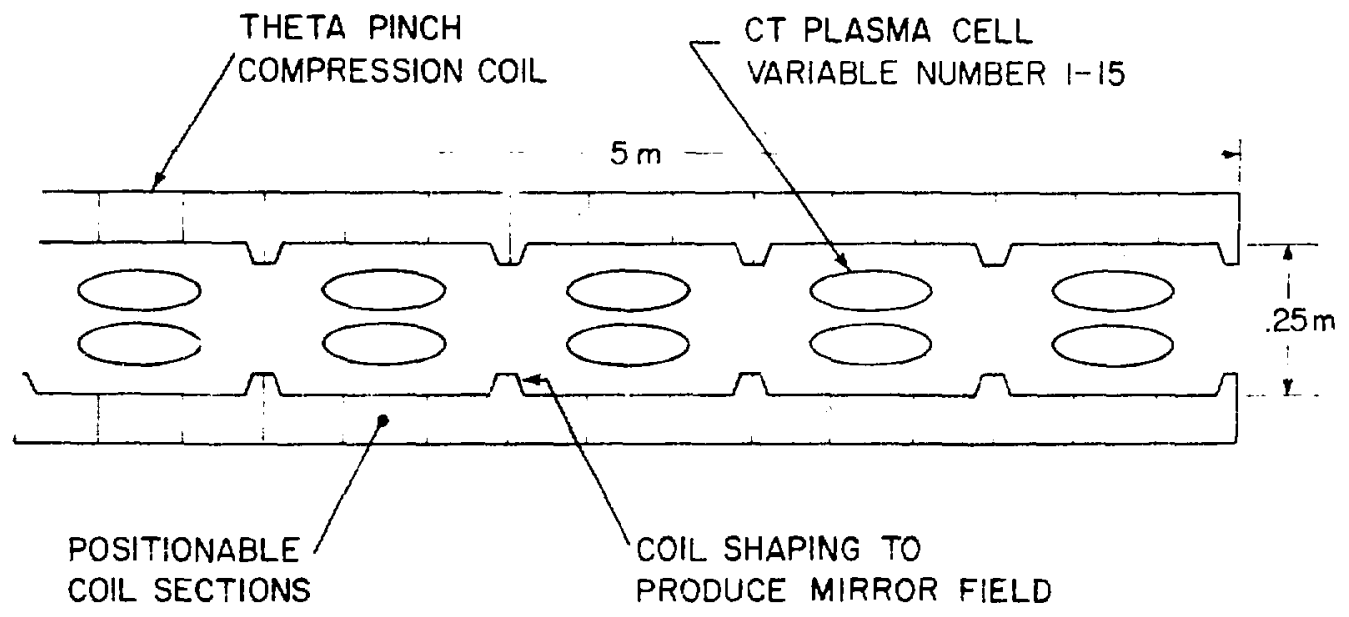

MULIIPLE-CELL EXPERIMENT

Fig. A-16.

Multiple-cell experiment on Scylla IV -P theta pinch.

64 


\section{Schedule and Manpower}

\section{A. Introduction}

Obviously the physics of CT's is not well established at the present time. Therefore, the PERT chart shown in Fig. A-17 is designed to answer as many key questions as possible as quickly as possible. An accelerated program at higher cost could be considered, but the assumptions made here are 1) the total CTR-Division resources grow at a modest rate, and 2) the CT program will not interfere with the major project in the LASL CTR-Division, viz., the Reversed Field Pinch, ZT-40 expertment. The unified application of remaining resources in the division yields the aggressive Program Plan developed in this document and summarized in Fig. A-17. It should be stressed that the experiments

\section{LASL COMPACT TORUS PERT CHART}

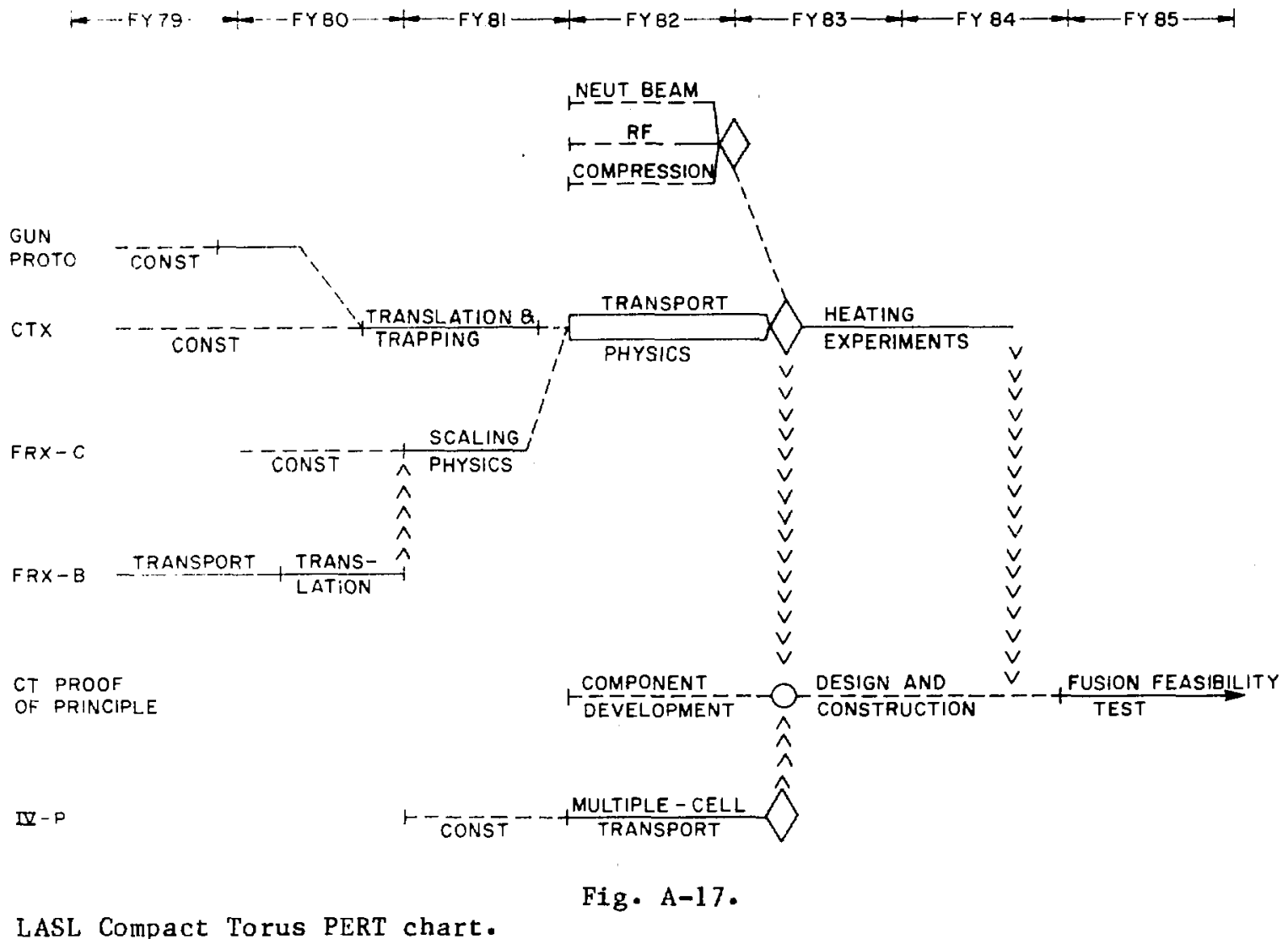


planned make good use of available hardware and require minimum major procurement for the next two fiscal years (FY' 80 and FY' 81 ). Experiments are carefully arranged during this time to permit adequate operational and diagnostic support while keeping an approximately constant level of design and construction as is consistent with the available engineering support. Assuming successful demonstration of the answers to physics issues defined above, the program grows in the years FY 1982-1985.

B. Pert Chart Logic

As discussed above in general, the single-cell CT investigations follow two main paths on Fig. A-17. The upper path concerns tori with toroidal field. It starts with the construction of the CTX facility and simultaneous production studies in the prototype gun experiment. These studies shift to translation, trapping, gross stability, etc, investigations on the CTX facility through August 1981. On the lower path, which is for the theta-pinch branch, transport and translation experiments continue on FRX-B while design and construction of the FRX-C experiment is underway. The FRX-C experiment is built in its final position as a source for the CTX facility. This results in a considerably more crowded space situation than if $F R X-C$ were built elsewhere, and there will be some interference between the operation of CTX and the construction of FRX-C for a few months. However, the overall savings in time and effort of not having to build two versions of FRX-C (or alternately of not having to move FRX-C) makes this plan necessary if the physics results of transport and heating are to be obtained on a timely basis. In October 1980 the FRX-B experiment stops, and FRX-C begins and rurs for about a year. In August 1981 the wall between CTX and FRX-C is removed, and FRX-C is connected to CTX with a short transition section. This takes only about two months which is the payoff for this crowding of the two experiments together. At this time the construction effort shifts to the multiple-cell experiment. Beginning in October 1981, single-cell transport physics is studied on CTX with two sources; a smaller crew does the multiple-cell transport physics on the modified Scylla IV-P facility. Also in October 1981 additional staff are acquired for the beginning of component development for a future Proof-of-Principle experinent and also to begiit the development of the heating hardware. In October 1982 a choice is made as to which should be the initial. heating method to be tried on CTX. 
In January 1983 two very important decisions are made for the whole program. At this time a choice is made as to which type of single-cell CT will be heated. Also, based on the comparison of the results from single-cell and multiple-cell transport studies, a provisional choice is made as to which cell configuration should be used in a follow-on, larger, fusion feasibility test. Input from the reactor studies which have been going on throughout this time will also bear on these two choices. Finally, the heating experiments conducted on CTX, provide information more or less continually for the design of the fusion feasibility test.

C. Manpower Projection

Using Fig. A-17 as a guide for requirements, it is seen that the major expansion occurs in FY 1982-1984 in preparation for a Proof-of-Principle Experiment. A preliminary estimate of the manpower needed to meet these requirements is given in Table $I$.

TABLE I.

MANPOWER ESTIMATE

SCIENTIF IC

SUPPORT

$\underline{\text { FY }}$

$\underline{\text { Staff }}$

$\underline{\text { Tech }}$

$\underline{\text { Staff }}$

Tech

1980

16

20

1981

17

20

1982

23

22

24

23

21

24

5

6

7

1983

1984

9

10

13

14

13

14 


\section{References}

1. A. E. Robson, "A Conceptual Design for an Imploding-Liner Fusion Reactor (LINUS)" NRL Memorandum Report 3861, September 1978.

2. A. C. Smith Jr., et al., "Preliminary Conceptual Design of the Moving Ring Field-Reversed Mirror Reactor," Pacific Gas and Electric Company 78 FUS-1, August 31, 1978.

3. G. A. Carlson, in "Mirror Fusion 1978: The Erice Lectures and Innsbruck Papers," Lawrence Livermore Laboratory, 1978.

4. H. Alfvén, et al., J. Nuc1. Energy, Part C: P1. Phys. 1,116 (190).

5. W. B. Jones and R. D. Miller, Phys. Fluids 11, 1550 (1968).

6. R. Turner, Phys. Fluids 132398 (1970).

7. D. R. Wells, et al., Phys. Rev. Lett. 33, 1203 (1974).

8. M. N. Bussac, et al., "Low Aspect Ratio Limit of the Toroidal Reactor: Spheromak," IAEA-CN-37X-1, Innsbruck, 1978.

9. W. Grossman, et al., Bull. Am. Phys. Soc. 22, 1189 (1977).

10. J. D. Sethian et al., Phys. Rev. Lett.1, 798 (1978).

11. C. W. Hartman, Mirror League Meeting, Livermore, March, 1978.

12. I. Henins and J. E. Hammel, Bull. Am. Phys. Soc. 22, 1115 (1977). 


\title{
APPENDIX B--REVIEW OF PREVIOUS EXPERIMENTS
}

\author{
R. E. Siemon
}

Early in the history of theta-pinch research the obvious advantages of a closed field line configuration were recognized. Every major laboratory that has reported theta-pinch experiments has devoted part of its effort to field reversed configurations. A brief recap of the major conclusions recorded in the literature is included here to help in formulating the key issues and defining the proposed experimental program.

Table B-1 summarizes parameters associated with various experiments. It is intended to give an overview and should be used in conjunction with the original references. The lifetime in the table is the best reported by the authors, and to the extent possible the plasma parameters are consistent with the stated lifetime. Lifetime for the quiescent phase is defined as the duration of a field-reversed configuration independent of why it is terminated. The main indicators are excluded flux or a hollow luminous structure observed from the end. The first indication of $n=2$ activity, when observed, is at a time generally somewhat shorter than the lifetime stated in the table.

A. Culham

Early experiments were reported by Bodin et al. ${ }^{1-3}$ using second half-cycle operation in which no control of the blas or preionization was possible. It was found that an important feature of reversed field operation is the generation of axially moving area waves or shocks leading to irreversible heating processes beyond those of implosion and compression. An axial contraction of the field-reversed configuration by typically a factor of three or so is a common observation in experiments. Following the axial contraction, the plasma lifetime was usually limited by an instability linked to rapid rotation. 3 According to end-on framing photographs the field reversed configuration disappeared before the onset of rotation. Typical parameters are listed in Table $\mathrm{B}-1$.

Later experiments by Green ${ }^{4,5}$ drew attention to means for extending the lifetime of the field-reversed configuration (from 2 us of earlier results to over $6 \mu \mathrm{s})$. The best confinement resulied when trapped field was minimized 


\begin{tabular}{|c|c|c|c|c|c|c|c|c|c|c|c|c|c|c|}
\hline & & 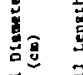 & $\frac{\bar{a}}{5}$ & $\widetilde{E}$ & $\frac{\widehat{g}}{3}$ & & 送 & g g & $\Xi$ & & & & & \\
\hline & 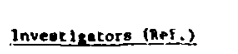 & $\overrightarrow{\mathrm{g}}$ & $z^{\prime}$ & $=$ & $\infty^{-1}$ & 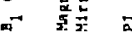 & $\bar{z} \mathrm{a}^{\circ}$ & $\varepsilon^{0}$ & $\therefore$ & 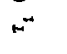 & $=$ & 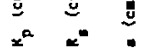 & 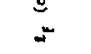 & 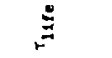 \\
\hline & & 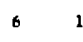 & 1000 & ss & $0-4$ & $6.7 \quad 1.2$ & $=80$ & $5.3 \times 10^{15}$ & 200 & 100 & $6 \times 10^{16}$ & & - & 3 wee \\
\hline & in et & 6.9 & 436 & 62 & & $6.4 \quad 1.5$ & 3 & & & -- & -- & -- & & 4 wee \\
\hline & & 5.4 & 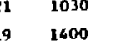 & $\begin{array}{l}86 \\
125 \\
125\end{array}$ & $\begin{array}{l}2.3 \\
2.12 \\
2.12\end{array}$ & & $\theta$ & & ${ }^{180-000}$ & & & - & 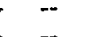 & \\
\hline & (areen (4) & 10 & $10 \quad 630$ & so & 0.5 & $\begin{array}{l}7.9 \\
4.8 \\
4.2 .0\end{array}$ & 255 & $\begin{array}{l}5.3 \times 10^{6} \\
3.6 \times 10^{15}\end{array}$ & $\begin{array}{l}800 \\
300\end{array}$ & $\begin{array}{l}1000 \\
300\end{array}$ & $\begin{array}{l}2 \times 10^{2} \\
20^{17^{10}}\end{array}$ & $=-$ & $\bar{z}$ r & 6 woec \\
\hline & Rostn (6) & 5 & 1235 & ${ }^{15-80}$ & $<5$ & $\begin{array}{l}2-6 \\
-6\end{array}$ & $=25-124$ & $1.6-7.9 \times 10^{10^{1.5}}$ & - & -- & $--_{17}$ & $r \quad \cdot$ & -- & \\
\hline & 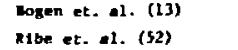 & io & $\begin{array}{l}100 \\
100 \\
10000\end{array}$ & $\begin{array}{l}60 \\
93 \\
93\end{array}$ & ${ }_{0-12}^{2}$ & $\begin{array}{l}8.9 \\
0.1 \\
0.1 .0\end{array}$ & $\begin{aligned} \theta & 230 \\
\theta & 30-12\end{aligned}$ & $\begin{array}{l}1.5 \times 1.20^{16} \\
3.38-2.2 \times 10^{15}\end{array}$ & $\begin{array}{c}600 \\
800\end{array}$ & 2000 & $\begin{array}{l}10^{17} \\
0 \times 10^{16}\end{array}$ & $\overline{1.5}=-$ & & 3 \\
\hline & Hense 6 Kolb (32) & 13.5 & 1801100 & 4 & $s$ & $2.0 \quad 1.0$ & $8 \quad 30$ & $3.3 \times 10^{15}$ & .. & -- & & $\ldots \ldots$ & & $25 \mathrm{suc} \cdot$ \\
\hline & Kolb & 10.5 & $180 \quad 260$ & 88 & 5.8 & $3.0 \quad 1.0$ & 9 & $60 x>2$ & 900 & 600 & $1.3 \times 10^{17}$ & 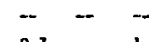 & & \\
\hline & 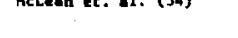 & 130 & 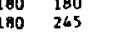 & 55 & 4.0 & $\begin{array}{l}2.6 \\
3.1 \\
3.1 .0 \\
1.0\end{array}$ & 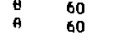 & $4.0 x$ & $\begin{array}{l}300 \\
200 \\
200\end{array}$ & $\begin{array}{l}\frac{300}{200} \\
200\end{array}$ & 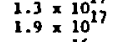 & & 160 & \\
\hline & 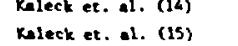 & 10.5. & & & 1.9 & $3^{1.6}$ & & & & & & & Bo & \\
\hline & & 10.2 & 92 & 27 & 0.64 & 3. & & $3 \times 10^{15}$ & $\ldots$ & & & & $0^{\circ}$ & \\
\hline & frosasenn (19) & $\begin{array}{l}\substack{10.5 \\
10.5 \\
10.5} \\
\text {. }\end{array}$ & $\begin{array}{ll}40 \\
40 \\
0\end{array}$ & $\begin{array}{l}28 \\
28 \\
28 \\
2\end{array}$ & $\begin{array}{l}0.8 \\
0.8 \\
0.8\end{array}$ & 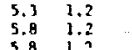 & 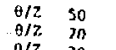 & 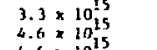 & $\begin{array}{c}60 \\
j 8 \\
j 8\end{array}$ & $\because$ & $\begin{array}{ll}4 & \times 10106 \\
6 \times 10^{16} & 16\end{array}$ & $\begin{array}{l}1.1 \\
1.0 \\
\text {. }\end{array}$ & 18 & 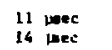 \\
\hline & & $\therefore 2$ & is & 4 & in & $\begin{array}{lll}\mathrm{NA} & x, 0\end{array}$ & 10-7 & $.066-6.6 \times 10^{15}$ & 20 & . & $6=10^{16}$ & $\begin{array}{l}1.6 \\
16\end{array}$ & $\begin{array}{lll}5 & 26 \\
-3 & 40\end{array}$ & \\
\hline & 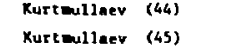 & ${ }^{20}$ & 90 & ${ }_{0,5}^{2}$ & $\begin{array}{l}1.0 \\
0.3\end{array}$ & $\begin{array}{l}\mathrm{BA}_{\mathrm{BA}} \\
\mathrm{BA}\end{array}$ & $\because 0.5$ & & $\begin{array}{l}1000 \\
300\end{array}$ & $\begin{array}{l}300 \\
70\end{array}$ & $\begin{array}{l}7 \times 10^{13} \\
2 \times x 0^{14}\end{array}$ & 10 & -32 & \\
\hline & Kurcteusisea & ${ }_{40}^{20}$ & & & $\frac{2}{2}$ & $* n$ & & $\left(\begin{array}{ll}1014 \\
1_{14}^{14}\end{array}\right.$ & $\begin{array}{l}200 \\
100 \\
100\end{array}$ & $\begin{array}{l}100 \\
100\end{array}$ & 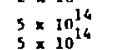 & 8 & 30 & \\
\hline & Inford et. & & & & 1.0 & & & $6.6 \times 10^{\prime}$ & 象 & 200 & $3 \times 10^{15}$ & & & \\
\hline
\end{tabular}

"Lifetion lialted by manetic field uavefors. 
because in that situation the axial compression which led to instabilities was minimized.

Bodin $^{6}$ reported experiments in which framing camera photography was used to identify an axial structure in the early formation of the field reversed configuration. Discrete rings were observed to be formed which then moved axially and sometimes merged into a contracted single cell. The rings were formed early in the implosion phase and were associated with resistive tearing modes that were fully developed in 0.2-0.5 usec. The theory for resistive tearing modes cited to agree with the experiment was given in a paper by Furth, Killeen, and Rosenbluth ${ }^{7}$ referred to as FKR theory.

An important limit on the maximum possible bias fleld was pointed out by Green and Newton. 8 Calculations and experiments were cited that show that the trapped bias is limited to a value $B=5 \times 10^{3} E_{\theta}\left(\alpha \quad p_{0}\right)^{1 / 2}$ where $B$ is the maximum initial bias, $E_{\theta}$ is the electric field at the discharge tube wall in $\mathrm{V} / \mathrm{cm}, \alpha$ the fraction of gas lonized, and $\mathrm{p}_{0}$ the initial deuterium presssure in mtorr.

B. Jül1ch

The Jülich group was among the first to develop techniques for independent preionization and bias.9-12 A noteworthy result of the early work was that Impurities played an important role in determining the lifetime.13 By discharge cleaning, the plasma lifetime could be extended to last for a complete half cycle $(1.8 \mu \mathrm{s})$, whereas rapid decay of trapped field and non-symmetric discharges were associated with higher levels of impurity. Also of interest in the early work was the paper by Kever ${ }^{12}$ which indicated that Implosion heating becomes less effective when the bias field exceeds a critical value. The value to within a multiplying constant is identical to the maximum bias derived by Green and Newton. 8

A very interesting experiment called Juliettal3,14 was reported in the late $60^{\circ} \mathrm{s}$. The diagnostics were mainly excluded flux and plasma streaks. Conclusions for parameters listed in Table $I$ were as follows:

1. Complex axial variations are observed presumably resulting from a resistive tearing mode (typically three or four plasma cells in a $1.3-\mathrm{m}$ coil). 
2. The tendency for tearing is reduced by reducing the magnetic compression following the inftial implosion.

3. The "fragments" of plasma that result from tearing appear to be stable and long-lived (particle containment in excess of $50 \mu \mathrm{s}$ ).

Based on the experimental results, the opinion was offered that the tearing mode was not a fundamental limitation since the resulting fragments were stable. Furthermore, weak magnetic mirrors should help in the confinement of the fragments which tended to move out of the ends of the coil.

\section{Garching}

Measurements by Keilhacker ${ }^{16}$ showed that quartz shielded probes placed inside a field reversed configuration led to localized cooling and resistive instabilities which rapidly destroyed the configuration. In a study of resistive tearing modes Eberhagen and Glaser 17 observed that either inserted probes or empty external side pockets (and the associated hole in the external coil) could induce the plasma to break into discrete plasma rings. The plasma formed with smooth walls was sometimes unstable, but could be free of the tearing mode if a discharge cleaning procedure was used. The major impurity problem was felt to be water deposits adsorbed on the quartz tube walls. It was also reported that the ring formation time appeared to be slow in comparison with the predictions of theory. In discharges free of the tearing mode ${ }^{18}$ time- and space-resolved density measurements showed that the axial contraction was consistent with a complete sweep up of the initial plasma.

A relatively complete characterization of a stable field-reversed configuration is given in the paper by Eberhagen and Grossmann. 19 It was found that an axially uniform single plasma cell was formed which decayed slowly until discuption by a rotational $n=2$ instability ended the confinement. Parameters are given in Table $B-1$. Possible explanations offered for the absence of tearing modes seen in earlier experiments were 1) a reduced level of initial plasma pèrturbation, or 2) a stabilizing effect from the observed plasma rotation. Shaped coils (mirror ratio 1.2) were found to eliminate a cendency for plasma to leave the coll axially. Before the disruption by $n=2$ there were no observable MHD instabilities although the excluded flux exhibited a gradual decay during the quiescent period of about $15 \mu \mathrm{s}$. It was also noted 
that under conditions of poor preionization the plasma exhibited strong axial inhomogeneities which could have been mistaken for tearing modes had they not been elimfnated by more efficient preionization.

The most recent Garching experiment was a Pololdal Field Belt Pinch. 20 The geometry of coll and vacuum vessel resembled a belt pinch but the windings were arranged to produce only a pololdal fleld. The aspect ratio (major to minor radius, $\mathrm{R} / \mathrm{a}$ ) was approximately 10 and as a result of the larger plasma moment of inertia it was possible to avoid the rotational $n=2$ instability. A resistive mode (separation Into two rings) limited the plasma Iffe, and it displayed a growth rate in good agreement with FKR theory. ${ }^{7}$ The plasma lifetime was about ten times longer than the characteristic growth time for MHD Instabilities but the authors caution that the relatively low temperatures $\left(T \sim 20 \mathrm{eV}\right.$ and $\left.\omega_{1} \tau_{1}<1\right)$ may lead to results inapplicable for fusion conditions.

\section{Naval Research Laboratory}

The early work by Kolb and colleagues $21-24$ was more concerned with heating than with confinement. In particular cases it was noted that large electric fields can arise at the field null in a field reversed experiment. 22 As a consequence neutrons can be produced by nonthermal acceleration of ions.

Models for plasma conflnement and loss processes in the theta-pinch with zero or parallel bias were determined to agree fairly well with experiment. In constrast, the factors which influence heating and confinement with an initial reverse field were stated to be complex and poorly understood. 24

A frequent experimental observation was the formation of two well-defined filaments that rotate with angular velocity $\Omega \sim 10^{7} \mathrm{sec}^{-1}$. The origin of the implied angular momentum was discussed in a paper by Rostoker and Kolb. 25 They argue that leakage of plasma cannot explain the rotation because l) a substantial fraction of the plasma would have to leak out of the ends, and 2) the predicted sign of rotation would be wrong. The explanation given assumes constant angular momentum during the dissipation of trapped fields, but it does not explain the origin of the initial value of angular momentum. 
The PHAROS experiments ${ }^{26-28}$ used a larger coil and demonstrated longer plasma lifetimes. Based on results with applied small transverse magnetic fields, it was established that rotation can be a result of transverse fields. 29-31 Hintz and Kolb ${ }^{32}$ reached the following conclusions:

1. Confinement limited by magnetic field duration (no crowbar) could be obtained with field reversed configurations, while zero or parallel bias fields led to more rapid plasma losses.

2. An axial contraction was observed for the fleld reversed case. Outside the central region a narrow thread of plasma connected the bulk of the plasma to the ends of the coll.

3. While rotation can in some Instances result from small transverse flelds, it remains unclear whether that explains rotation in the cases where the coil and feed plate arrangement limited the stray fields to less than one percent.

4. The rotational velocity and $\mathrm{n}=2$ instability growth rate increase with larger transverse fields and decrease with larger current density or larger electron temperature.

With an extended current puj. $2^{35}$ it became clear that plasma confinement was not limited by the magnetic field duration. With the highest temperatures, confinement was limited by the onset of $n=2$ instabilities and not by particle 1oss.

Detailed measurements of plasta particle loss were presented by Mclean et al.34 Two coil diameters were compared. The higher temperature (small bore) confinement exceeded Bohm by more than an order of magnitude. The lower temperature (larger bore) showed improved confinement during a quiescent phase after which plasma was lost rapidly at roughly a rate consistent with Bohm. The axtal contraction and other features of field reversed operation were in good qualitative agreement with the other laboratories.

In an attempt to improve confinement further PHAROS was converted to a hard-core experiment. ${ }^{36-39}$ The results were of limited success primarily because of a complex instability eventually associated with a helical structure following current paths in the plasma. 39 The limits for confinement of the observed multhelix plasma were never established because of the limited duration of the applied magnetic fields. 
Axial irregularities, posstbly associated with the development of resistive instabilities in other experiments, were given a new interpretation by Dixon, Düchs, and Elton. 40 Their evidence shows that the structure originates with the initial breakdown phase of the preheater discharge. A theory by Haines 41 for instabilities associated with thermal waves is proposed to explain the results.

\section{E. Kurchatov Institute (Krasnaya Pachra)}

The first work by Kurtmullaev on field-reversed configurations was reported in two papers by Es'kov et al.42,43 at the Sixth European Conference in 1973. The experiment 42 demonstrated that the axial shock formed after reconnection is an effective heating mechanism. A metal disk (perpendicular to the axis) Inside the discharge tube at one end of the external coll was used to speed the reconnection process. The axial shock propagating away from the disk increased the plasma pressure by a factor of ten over the value predicted for radial implosion alone. The second paper 43 was a proposal to imbed toroidal field in the intial plasma with an axial current and then form a field-reversed configuration with rapid increase of the pololdal ( $\theta-p i n c h$ ) field.

Experimental results with and without toroldal fields were reported in 1974.44 It was found, as in other experiments, that the "1ifetime without toroidal field is at least an order of magnitude greater than expected, evidently ". - related to pecularities in the behavior of the magnetic field in toroidal geometry with an elongated cross section." Plasma confinement was limited to about ten microseconds because of a rather poor crowbar. Preliminary results with embedded toroidal field from a hard-core shielded conductor indicated: 1) the conditions for shaping and heating the toroidal plasma are noticeably different as a consequence of plasma interacting with the structure on axis, and 2 ) it is possible to create a closed toroidal field of prolonged existence.

A solution to the problem of limited bias fleld (Green and Newton, Ref. 8) was reported at Lausanne. 45 Longitudinal conductors parallel to the axis outside the discharge tube are pulsed to form a barrier octopole field during the phase when the applied field begins to rise. In this way the expansion of 
plasma against the walls of the vacuum vessel is inhibited and larger bias field can be employed. Probe measurements indicated a significant increase in the trapped flux. Within the limits imposed by a poor crowbar it was concluded that the confinement time was as much as $50 \mu \mathrm{sec}$ under those conditions where the plasma diameter to coil diameter ratio was 0.5 to 0.8 . A diffuse current sheet was associated with the long confinement time. Parameters, given in Table I, were obtained with a delayed crowbar and a fairly low magnetic field.

A discussion of the experiments was also presented in a 1976 report. 46 An introductory comment was the following: "For the purpose of stabilization one can introduce toroidal magnetic fleld. However, at the present $t$ ine promising results were obtained concerning stability of such structures in the absence of this component of the magnetic field." It appears that while theoretical considerations have motivated the experimenters to introduce toroidal fields, the experiments thus far have not shown any resulting improvements. The report offers many observations and conclusions:

1. Heating by an axial moving shock increases the temperature by a factor $(\ell / R)^{2}$ compared to the radial Implosion, and this amounts to a factor of 10-30 in typical experimental arrangements.

2. The geometry of field-reversed systems makes it possible to transport the plasma toroid and thereby spatially separate the region of formation and heating from the fusion blanket region.

3. Experimentally the longest stable confinement is for ratios of plasma radius to wall radius of order unfty. Strong radial compression after the closed configuration is formed leads to losses of particles, magnetic flux, and energy.

4. A rapidly pulsed barrier field (Ioffe bars) is an effective way of reducing plasma losses during the time when the applied poloidal field is being reversed. The consequence of increasing the trapped flux is a general tendency toward stability associated with the increase in radial dimensions.

5. Calculations for the proposed case of embedded toroldal field indicate that significant heating and high beta are consistent with stable equilibrium ( $q>1$ ) formed with embedded toroidal field.

6. Calculations are presented which describe the equilibrium of a highly elongated torold without embedded toroldal flelds and the heating to be expected from the axial shock compression. It is corcluded that the 
maximum shock heating occurs when the inftial current layer of thickness $\delta$ is small with respect to the separatrix radius.

7. For experimental parameters, the axial contraction time is comparable to a sonic transit time, and therefore neither shock heating nor an adiabatic compression description is strictly applicable.

8. The present work addresses and solves the following fundamental problems: a) pulsed formation of a compact toroidal plasma

b) shock heating of the plasma by longitudinal compression with the aid of poloidal field

c) relaxation of shock perturbations without losses in the presence of stable confinement

d) inhibition of the radial particle and magnetic flux losses and the prevention of MHD instability in the inversion phase of the field.

In the Innsbruck paper 47 there are a number of new developments reported. A significant Improvement in confinement time ( $\tau \sim 100 \mu \mathrm{sec})$, a demonstrated method of transporting plasma torolds, and refinements in controlling the field reconnection at the ends are the major points.

With regard to field reconnection, it was found possible in experiments to greatly delay tearing at the ends (up to $40 \mathrm{usec}$ ). During that time plasma was confined radially by the barrier fields and axially by steady-state mirror coils at the ends of the shock coil. Also during that time the reversed field was applied on a relatively slow time scale. Then a fast rising "trigger coil" permitted rapid reconnectionn to be inftiated at the ends with the subsequent development of a strong axial shock. Without the trigger coll the axial shock was more diffuse and weak. The prolonged life of a fleld-reversed but open-ended configuration is thought to differ from all previous experiments because of the barrier field which maintained the plasma several centimeters from the material walls. This wall-plasma isolation was found benefictal in suppressing tearing modes. Tearing was observed to occur under certain conditions such as near x-ray targets inserted into the discharge tube or when the bias field was initially weak. It was also established that perturbations in the process of developing could be stabilized by the action of the longltudinal shock and compression.

The advantages in a fusion reactor to be gained by separating the high-voltage formation and heating region from the fusion region require transporting a plasma toroid. A method of accomplishing the transfer from the 
shock coil to a quasi-steady magnet was reported. The "trigger coil" used to initiate an axial moving shock was activated at only one end of the shock coil. The subsequent shock moved away from the trigger coil, and providing the timing was adjusted to synchronize with the diffusive reconnection at ihe other end, the result was a plasma toroid which moved axially at roughly the poloidal Fiston velocity $\left(\sim 10^{7} \mathrm{~cm} / \mathrm{sec}\right)$.

Confinement was found to be limited by the crowbar time $t \sim 100$ Hsec. No sign of instability was observed, and it was stated that no particje loss or energy loss other than adiabatic decompression were measurable during the time of observation. Lnfortunately these very encouraging results were not documinted very completely with data in the paper. Interferometric donsity neasurements were given but the magnetic field waveform and geometry werc not included for tise conditions of the measurement. Table B-1 assumes the nomiral paraneters listed elsewhere for the coil with $8.8 \mathrm{~cm}$ radius. The good results were found to be cricically dependent on the ratio of separatrix ridius to wall radius. It was ctseryed that the separatrix radius must exceed a valut at 2.5 to $3.5 \mathrm{~cm}$ for a wail radius of $8.8 \mathrm{~cm}$. This was possible far initial pressures ranging from appeoximately 2-ljumtorr. On the other hand, filling pressuru wutside this range leads to a sharp decrease in confinement (t 510 , sec) : It was concluded that a diffuse current sheet with thickness romajable tu tive separatrix radius is optimum for stability and confinement.

F. Los $k$ lamos

Farly experiments with reversed bias fields were motivated by heating questions. 48-54 It was observed that fast compression of preionized plasma with an embedded reverse bias field emitted neutrons, whereas zero or positive bias did not. This effect was understood as an enhanced ion heating during the implosicn. Subsequently it was determined that lower filling presssure without a bias permitted heating and neutron emission 53 while avaiding the various complexities assocfated with reversed bias such as the $n=2$ rotational mode. The standard Los Alamos theta-pinch configuration became the zero-bias mode and the Scyllac project utilized that mode experimentally and assumed it in the theory. 
Recent experiments by Linford et a1.55-59 have reexamined the potentials of confinement in the fleld reversed configuration. Dlagnostics include external and internal magnetic probes, streak and framing cameras, spectroscopy, interferometry, and pressure probes. The parameters listed in Table $B \rightarrow 1$ were reported at Innsbruck. 55

Termination of the confinement usually follows the development of an $n=2$ mode. Direct evidence linking the instability to a gradually acceierated plasma rotation is provided by measuring the CV Doppler shift. The instability begins when the rotational velocity reaches about 0.4 of the diamagnetic $\mathrm{drift}$ velocity. Theoretical estimates by Freidberg and Pearlstein ${ }^{60}$ and Seyler 61 show that the deuterlum velocity should exceed the diamagnetic drift speed by a factor of 1.3 to 1.7 before instability occurs. Since the theories correctly predict the observed frequency of $n=2$ motion, it is now believed that the $C V$ rotation is an underestimate of the deuterlum plasma rotation.

Interferometry, luminosity measurements, and pressure probes sensing the flow of plasma on open field lines, all indicate that a significant plasma pressure exists on the fleld line separatrix and the nearby open field lines. This feature is mentloned only brlefly, if at all, in other published work. Together the data indicate that plasma is confined on closed field lines for a time considerably langer than the expected confinement on open lines, but the loss rate is finite and leads to a steady flow of plasma out of the system during the qufescent phase. The estimated particle confinement time associated with the parameters in Table B-l is 50-80 usec according to the pressure probe measurement.

G. University of Maryland

Experiments on the Maryland high voltage theta pinch ${ }^{62}$ are frequently performed using a reverse bias field. Since the magnetic field has a half-period of about $0.6 \mu \mathrm{sec}$, the time of observation is too limited for an estimate of plasma lifetime. However, Chen 63 reports neutron emission measurements which indicate significantly reduced plasma loss in the field reversed configuration. 
A paper by Hess and Griem ${ }^{64}$ reports that the plasma beyond the ends of the coil is heated too rapidly to be explained by classical energy transport mechanisms. The data were taken with a fleld reversed configuration and pose a question not yet resolved. The role of convective energy transport may be important but was not determined in the experiment.

Irby, Drake, and Griem $^{64}$ have recently reported very interesting observations of tearing mode activity in the field reversed mode. Thorough mapping of the internal magnetfc ffeld displays magnetic islands formed on a time scale of an Alfven transit time. A theoretical interpretiation is given which points out 1) the FKR theory of tearing modes ${ }^{7}$ does not apply because the fleld reversed theta pinch has no shear, and 2) slab models, often used to calculate resistive mode growth rates, are not applicable to an experiment in which field lines in the viclnity of a field null are essentially circular. A g, rowth rate is derived assuming magnetized electrons

$$
\gamma=\frac{\Omega_{i}}{\left(a / r_{i}\right)^{5 / 2}} \quad, \quad \frac{z}{r_{1}} \gg 1
$$

where $\gamma$ is the growth rate, $\Omega_{1}$ is the ton gyrofrequency, a is the scale length for field curvature, and $r_{1}$ is the fon larmor radius. The calculation is in reasonable agreement with experimental observation. The nonlinear evolution and apparent saturation in other ffeld reversed experiments is receiving further study. 


\section{References}

1. H. A. B. Bodin et al., Nucl. Fusion: 1962 Supplement, Part 2, 511 (1961).

2. H. A. B. Bodin et al., Nucl. Fusion: 1962 Supplement, Part 2, 521 (1962).

3. H. A. B. Bodin and A. A. Newton, Phys. Fluias 6, 1338 (i963).

4. T. S. Green, Phys. Fluids $\underline{6}, 864$ (1963).

5. T. S. Green, Nuc1. Fusion 3. 57 (1963).

6. H. A. B. Bodin, Nucl. Fusion $\underline{3}, 215$ (1963).

7. H. P. Furth, J. Killeen, and M. N. Rosenbluth, Phys. Flu1ds $\underline{6}, 459$ (1963).

8. T. S. Green and A. A. Newton, Phys. Fluids 9. 1386 (1966).

9. H. L. Jordan, Nucl. Fusion: 1962 Supplement, Part 2, 589 (1962).

10. H. Beerwald et al., ibid, 595.

11. E. Hintz, 1bid., 601 .

12. H. Kever, ibid., 613.

13. P. Bogen, E. Hintz, J. Schlüter, Nuc1. Fusion 4, 131 (1964).

14. A. Kaleck et al., Proceedings of the APS Topical Conference on Pulsed High Density Plasmas, Los Alamos Scientific Laboratory report LA-3770, A4-1 (1967).

15. A. Kaleck et a1., Plasma Physics and Controlled Nuclear Fusion Research, Novosibirsk (1968), 581, (IAEA, Vienna 1969).

16. M. Kellhacker, Nuc1. Fuston 4 , 287 (1964).

17. A. Eberhagen and H. Glaser, Nuc1. Fusion 4, 296 (1964).

18. A. Eberhagen and H. Glaser, Z. Naturforschung 20a, 1268 (1965).

19. A. Eberhagen and W. Grossmann, Z. Physik 248. 130 (1971).

20. G. Becker et al., Plasma Physics and Controlled Nuclear Fusion Research 1974, p. 47, Fifth Conference Proceedings (Tokyo), (IAEA, Vienna) 1975.

21. A. C. Kclb, Proceedings of the Second United Nations International Conference on the Peaceful Uses of Atomic Energy, Geneva, 1958 (United Nations, Geneva, 1958) Vol. 31, p. 328. 
22. A. C. Kolb, C. B. Dobble, and H. R. Griem, Phys. Rev. Letters 3 , 5 (1959).

23. A. C. Kolb, H. R. Griem, and W. R. Faust, Proceedings of the Fourth International Conference on Ionization Phenomena in Gases, R. Nilsson, Editor (North-Holland Publishing Company, Amsterdam, 1959), Vol. IV C, p. 1037 .

24. A. C. Kolb, Rev. Modern Phys. 32, 748 (1960).

25. N. Rostoker and A. C. Kolb, Phys. Rev. 124, 965 (1961).

26. H. R. Griem et al., Nucl. Fuslon: 1962 Supplement, Part 2, 543 (1962).

27. A. C. Kolb et a1., Nucl. Fusion: 1962 Supplement, Part 2, 553 (1962).

28. K. Hain and A. C. Kolb, Nuc1. Fusion: 1962 Supplement, Part 2, 561 (1962).

29. M. G. Haines, Phys. Letters 6 , 313 (1963).

30. E. Hintz et al. Phys. Fluids I, 153 (1964).

31. P. C. Thonemann and A. C. Kolb, Phys. Fluids $\underline{8}, 1455$ (1965).

32. A. C. Kolb, P. C. Thonemann, and E. Hintz, Phys. Flulds $\underline{8}, 1005$ (1965).

33. E. Hintz and A. C. Kolb, Phys. Flu1ds 8, 1347 (1965).

34. E. A. Mclean, A. D. Anderson, and H. R. Grlem, Proceedings of APS Topical Conference on Pulsed High-Denslty Plasmas, Sept. 9-12, 1967, Los Alamos Scientific Laboratory report LA-3770, p. A5-1.

35. A. C. Kolb et al., Plasma Physics and Controlled Nuclear Fusion Research, Vol. I, p. 261, (IAEA, Vienna, 1966).

36. A. C. Kolb, M. P. Young, and E. A. McLean, Proceedings of APS Topical Conference on Pulsed High-Density Plasmas, Sept.9-12, 1967, Los Alamos Scientific Laboratory report LA-3770, p. A5-1.

37. A. C. Kolb et al., Plasma Physics and Controlled Nulcear Fusion Research (IAEA, Vienna, 1969), Vol II, p. 567.

38. D. Düchs, R. H. Dixon, and R. C. Elton, in Proceedings of the Second Topical Conference on Pulsed High-Beta Plasmas, edited by W. Lotz (Institut für Plasmaphysik, Max-Planck-Institut, Garching, West Germany, 1972), p. 111 .

39. D. Düchs, R. H. Dixon, and R. C. Elton, Phys. Fluids $\underline{17}, 124$ (1974).

40. R. H. Dfxon, D. F. Düchs, and R. C. Elton, Phys. Fluids 16, 1762 (1973). 
41. M. G. Haines, J. Plasma Physics 12,1 (1974).

42. A. G. Es'kov et a1., Proceedings of the Sixth European Conference on Controlled Fusion and Plasma Physics, Moscow, USSR, 30 July-4 August, 1973, Vol I, p 599.

43. A.G. Es'kov et al., ibid, p. 595.

44. A. G. Es'kov et al., Plasma Physics and Controlled Nuclear Fusion Research 1974, Fifth Conference Proceedings, Tokyo, 1974 (IAEA, Vienna, 1975) p. 155 .

45. A. G. Es'kov et al., Proceedings of the Seventh European Conference on Controlled Fusion and Plasma Physics, (Lausanne, 1975) Vol I, p. 55.

46. A. G. Es'kov et al., IAE-2750, Kurchatov Institute, Moscow, USSR, 1976 (UCRL-Translation-11290, W. Sadowski, 1977).

47. A. G. Es'kov et al., Proceedings of the Seventh IAEA Conference on Plasma Physics and Controlled Thermonuclear Research, Innsbruck, 1978 (to be published).

48. E. M. Little, W. E. Quinn, and F. L. Ribe, Phys. Fluids 4, 711 (1961).

49. R. C. Mjolsenss, F. L. Ribe, W. B. Riesenfeld, Phys. Fluids 4, 730 (1961).

50. E. M. Little and W. E. Quinn, Phys. Fluids 6, 875 (1963).

51. F. C. Jahoda and G. A. Sawyer, Phys. Fluids $\underline{6}, 1195$ (1963).

52. F. C. Jahoda et al., J. Appl. Phys. 35, 2351 (1964).

53. E. M. Little, W. E. Quinn, and G. A. Sawyer, Phys. Fluids $\underline{8}$. 1168 (1965).

54. G. A. Sawyer et al., Phys. Fluids 10,1564 (1967).

55. R. K. Linford et

Proceedings of the Seventh IAEA Conference on Plasma

Physics and Controlled Thermonuclear Research, Innsbruck, 1978 (to be published).

56. D. A. Platts, R. K. Linford, and K. S. Thomas, Bull. Am. Phys. Soc. 21, $1074(1976)$.

57. D. A. Platts et a1., Bull. Am. Phys. Soc. 22, 1191 (1977).

58. R. K. Linford et a1., Bull. Am. Phys. Soc. 22, 1191 (1977).

59. R. K. Linford et a1., Bull. Am. Phys. Soc $\underline{23}, 781$ (1978).

60. J. P. Freidberg, L. D. Pearlstein, Phys. Fluids 21, 1207 (1978). 
61. C. E. Seyler (to be published).

62. A. W. Desilva, D. F. Düchs, G. C. Goldenbaum, H. R. Griem, E. A. Hintz, A. C. Kolb, H.-J. Kunze, and I. M. Vitkovitsky, P1asma Physics and Controlled Nuclear Fusion Research, Novosibirsk, 1968. (IAEA, Vienna, 1969), Vol. I, p. 143.

63. Y. G. Chen, Phys. Rev. Letters 37,703 (1976).

64. R. A. Hess, H. R. Griem, Phys. Fluids $\underline{18}, 1056$ (1975).

65. J. H. Irby, J. F. Drake, H: R. Griem, Phys. Rev. Lett. $42, .228$ (1979). 
APPENDIX $C$

D. C. Barnes and C. E. Seyler

A number of field reversed theta pinch experiments have demonstrated the formation of a reversed field configuration by a fast tearing mode followed by a quiescent phase in which no gross instabilitites are observed. This quiescent phase is later terminated by an $n=2$ rotationally driven mode. Motivated by these experimental observations theoretical work has concentrated on four major areas: formation and equilibrium; stability of a static (nonrotating) configuration; stability of rotationally driven modes; the origin and scaling of the spin up time to the onset of rotational instability.

A complete description of the formation of a fleld reversed configuration by rapid compression and reconnection of magnetic field lines requires a physical model wich includes single particle effects for the plasma ions. The hybrid models with massless electrons recently developed by Sgro and Nielsonl and by Hewett and Sgro ${ }^{2}$ includes all ion orbit effects as well as resistivity and thermal conduction for fluid electrons. One-dimensional simulations of the formation of field-reversed configurations have been carried out ${ }^{3}$ and the results of the FRX experiments have been qualitatively reproduced in the simulations. Two-dimensional ( $r, 2)$ calculations are necessary to correctly represent reconnection and axial contraction during the formation phase and this work is in progress.

A simpler description of the gross features of the quiescent configuration is obtained by considering single fluid, scalar pressure MHD equilibria. These are obtained as solutions to the Grad-Schafranov equation

$$
r \frac{\partial}{\partial r} \frac{1}{r} \frac{\partial \psi}{\partial r}+\frac{\partial^{2} \psi}{\partial z^{2}}=-4 \pi r^{2} p^{-}(\psi)
$$

where $\psi$ is the magnetic $f$ lux and $p(\psi)$ is a suitably chosen function. Anderson ${ }^{4}$ has numerically obtained solutions to the above which agree well with the plasma parameters produced in the FRX experiments by choosing $p(\psi)$ and the external boundary conditions in an appropriate way. 
Using a slightly more complicated numerical scheme, Grossmann and Hameiri 5 have determined $\psi$ and $p(\psi)$ consistent with adiabatic constraints appropriate to quasi-static time varying boundary conditions. These calculations determine the relationship between radial and axial contraction and allow one to investigate adiabatic compression and transport in field reversed configurations.

An even simpler model of equilibrium is provided by considering the plasma to be a sharp boundary, skin current equilibrium. Models of this sort have been developed by $\mathrm{Kadish}^{6}$ and independently by Linford ${ }^{7}$. The later model predicts the relation between the plasma major radius, $R$, minor radius, $a$, separatrix radius, $r_{s}$, wall radius, $r_{w}$, and half length, $b$.

A major conclusion ${ }^{7}$ from the sharp boundary equilibrium model is that a large compression of the plasma (large $r_{w} / r_{s}$ ) produces a plasma with a large inverse aspect ratio, $a / R$, and $a$ small elongation, $b / a$. The diffuse calculations have generated elongated equilibria and verified the predicted scaling of $b / a$ with $r_{w} / r_{s}$. The $a / R$ scaling with compression is more complicated since this $d$. sends on the profile chosen. In addition, the axial profile of the diffuse solutions tends to be much more Gaussian in shape than the trapezoidal like variation observed experimentally. Both the aspect ratio scaling and the axial shape are currently being studied.

A most surprising feature of field reversed experiments is the lack of unstable MHD modes. Recent theoretical work has been directed toward understanding these modes in a stationary field reversed configuration without toroidal field. Several classes of modes have been identifled and analyzed. These are: axisymmetric modes; gross displacements; small $n$ modes; and. large $n$ modes, where $\mathrm{n}$ is the turoidal (azimuthal) mode number.

For axisymmetric $(n=0)$ modes, an examination of $\delta W^{8}$ shows that stability depends on the pressure profile $p(\psi)$. A sufficient condition for $n=0$ stability to fixed boundary modes is $\mathrm{p}^{-\infty}<0.9$ This is difficult to realize in all of the plasma region with realistic boundary conditions but much less restrictive conditions should be sufficient as well.

The most dangerous free boundary $n=0$ mode is a gross displacement of the plasma along the major axis. For elongated configurations, the applfed axial field generally has a maximum in the midplane of the device and this axial mode will be unstable in the absence of an outer conducting wall. A conducting wall placed at a moderate distance from the plasma stabilizes this mode. It may 
also be possible to stabilize this motion by placing mirrors at the ends of the device but other instabilities limit the strength of these mirrors.

A second possible gross motion of the plasma is a sideways displacement of the plasma perpendicular to the major axis. This $n=1$ mode is similar to unstable Scyllac modes in bumpy and helical systems. A stralghtforward calculation shows that because, of the internal field reversed plasma ring, this sideways mode is normally stable and is destabllized only if strong external mirrors are applied at the ends of the device. Thus, there may be a stability window in which end mirrors stabllize axial translation without a conducting shell but do not destabilize the $n=1$ sideways mode.

For more general $n=1$ modes such as tilting and for $n>1$ but finite, large scale numerical methods are required. In three-dimensional MHD calculations ${ }^{4}$ using the MALICEl0 simulation code, all of the modes discussed above as well as large scale modes with $n \leq 3$ are well resolved and equilibria which are completely stable to all such modes have been constructed. These calculations have been carrled out for equilibria with plasma pressure on open field lines outside the separatrix. A major conclusion is that a field reversed configuration can be unstable to an $n=2$ flute like mode if the plasma is overcompressed. This occurs elther because the equilibrium changes as the plasma is moved farther from the wall or because wall stabilization is lost (both effects occur simultaneously in the calculations). Finally, $n=0$ or 1 modes (Including tilting) are always found to be stable in these simulat lons.

The above stability considerations explain the observed good gross stablitty of fleld reversed plasmas. To complete the MHD stability picture, localized modes are also considered. These local modes may be 1mportant for transport discussed below and may impose a lower limit on finite gyroradius size. Local modes correspond to the $11 \mathrm{mit} n+\infty$ and may be considered using the well known energy principle. There are two classes of these modes for a configuration which is symmetric about the midplane, even and odd modes. Within each class, the poloidal mode number, $m$, counts one half the number of nodes around a giver. fleld line.

The $\mathrm{n}=0$ mode (which is even) is a local interchange mode for which the normal displacement of a field line is approximately constant along the line. This mode is the analog of the $n=0$ sausage mode in a straight, cylindrical z-pinch and may be stabilized by a proper cholce of the pressure profile. 
Interchange stability for tiese zero shear configurations depends on the presence of closed field lines. Thus, the situation is quite different from that in devices with significant toroidal field. Interchange stable solutions have been constructed using analyticll and numerical ${ }^{5}$ techniques. These configurations are elongated and have finite pressure on or near the separatrix.

The $n=1$ local kink occurs either as an axial (odd) or a radial (even) translation of the field lines. Both of these modes are unstable for an elongated Hill's vortex, which is the only equilibrium investigated so far. The axial mode is the least dangerous of these two. For this mode the growth rate scales inversely with the elongation and the mode becomes stable for toroidal mode numbers smali compared to the elongation. The growti rate of the radial mode, which resembles a theta-pinch flute mode, is also inversely rolated to the elongation but this node may be unstable at small toroidal mode numbers as indicated by ti:e three-dimensional calculations described previously, Finice gyroradius effects on both of these modes are significant and probably supress them experimentally.

Finally, : itwcomb ${ }^{12}$ has slown that all $m>2$ modes are stable for the Hill's vortex and it is expected that this is generally true. A complete scudy of these lucal modes and finite gyroradius effects is underway.

In the prasent field reversal experfments it is rotational instability which terminates plasma confinement. 13 The rotational stability of infinitely long, Firld revorsed theta pinches has been studied by Pearlstein ${ }^{14}$, Freidberg ${ }^{15}$, and Seyler ${ }^{16}$. Freidberg used the finite Larmor radius equations in which he treated the field null in an ad hoc manner. The predicted value of the critical rotation velocity was about $\Omega \simeq 1.6 \omega_{\star}$, where $\omega_{\star}$ is the ion diamagnetic drift frequency. Using the exact Vlasov-Fluid equations in which no field null singularities occur, Seyler found the critical rotation velocity to be somewhat lower, $s \simeq 1.3-1.5 \omega_{*}$, depending upon the profiles. Both results, however, are in qualitative agreement, the threshold for $n=2$ rotational instability is $\Omega>\omega_{*}$.

The mechanism which causes the plasma to spin up to this instability threshold has not been positively identified. However, three types of explanations have been proposed. First, the plasma may initially rotate and because of profile changes related to transport later reach the critical rotation. The magnitude of initial rotation for this explanation to hold 
requires the ions to carry a large fraction of the current initially. Since this is inconsistent with a number of theta-pinch experiments, this explanation seems unlikely.

A second possible explanation of plasma rotation might be end shorting on the open field lines and viscous transfer of rotation to the remainder of the plasma. This is unlikely to be the full explanation because the energy avallable in the open field region is much less than the final rotational energy of the plasma.

Finally, particle and flux loss from the closed field region might result in the loss of angular momentum from the system and corresponding plasma rotation. This appears to be the most likely cause of rotation. There are five types of particle orbits in a field reversed configuration and only those encircling the axis or outside the separatrix have negative angular momentum. These particles are lost in a thermal transit time, resulting in a net gain of angular momentum by the confined plasma. In addition, resistive decay of the closed field flux corresponds to a net gain of mechanical angular momentum by the plasma. Thus, both of these effects are consistent with tiue observed rotation of the plasma.

The rotational velocity reaching the instability threshold requires the loss of one half to two thirds of the plasma. Classical cross field transport times are much too long and have the wrong scaling with temperature.

Anamalous transport may come about either from fluid (MHD) turbulence or from microturbulence. The instability of local MHD modes suggests the possibility of fluid turbulent transport but this is probably unimportant in the present experimental regime. This local MHD activity is supressed by finite gyroradius effects. Thus, transport from this source should increase when the ion gyroradius, $\rho_{f}$, is reduced. Experimentally, the opposite occurs ${ }^{13}$, transport worsens as $p_{1} / a$ increases.

Microturbulence can explain the observed confinement times in the present small experiments. Hamasak $1^{18}$ has obtained reasonably good agreement with the experimental observations using lower hybrid drift anamolous resistivity and including radial geometric effects. In future experiments and reactor type plasmas, the transport should be much more nearly classical. In this regime, the universal drift instability needs to be investigated. Finite $\beta$ effects may stabilize this tnstability but the effects of field line curvature may also be important. 
These rotation and transport considerations suggest that the rotational instability occurs only after a large part of the plasma energy is lost. Thus, the basic limitation on plasma confinement is particle loss. Two possibilities for improving particle confinement are suggested by our present experimental and theoretical understanding. First, cross field losses should be reduced by increasing $a / \rho_{1}$, 1.e. increasing the slze of the configuration. Second, plasma on the open field lines might be confined for many transit times, providing significant pressure on the separatrix and again reducing cross field losses.

A field reversed multiple mirror experiment would achieve this by converting the streaming loss of these particles to a multiple mirror diffusive loss for which the open field line confineruent would scale as 18

$$
\tau=t^{2} / e_{z}^{2}
$$

where $t=t_{1}+t_{2}, t_{1}$ is the average $t$ ime an ion is trapped between cells and $t_{2}$ is the time between trapping, $l_{z}$ is the distance between cells, and $L$ is the total length of the device. 
References

1. A.G. Sgro and C. W. Nielson, Phys. Fluids, 19, 26 (1976).

2. D. W. Hewett, et al., Bull. Am. Phys. Soc, 22, 1176 (1977).

3. A. G. Sgro, private communication.

4. D. V. Anderson and D. C. Barnes, LLL internal memo, MFE/TC/78-340.

5. W. Grossman and E. Hameir1, Paper 2B9, Proc. Theoretical Aspects of Controlled Thermonuclear Research, (1979).

6. A. Kadish, private communication.

7. R. Linford, private communication.

8. I. B. Bernstein, et al., Proc. Roy. Soc. A., 244, 17 (1958).

9. D. Dobrote and M. S. Chu, Phys. Fluids, 161371 (1973).

10. J. U. Brackbil1, Meth. In Con. Phys., 16, 1 (1976).

11. D. C. Barnes and C. E. Seyler, LA-UR-79-13 (1979).

12. W. A. Newcomb, private communication.

13. R. Linford, et al., FRX experiments.

14. L. D. Pearlstein, et al., Paper No. CN-37-5-1-2, Proc. Seventh International Conference on Plasma Physics and Controlled Nuclear Fusion Research, Innsbruck, Austria (1978).

15. J. P. Freidberg, private communication.

16. C. E. Seyler, submitted to Phys. Fluids.

17. S. Hamasak1, Paper 1841, Proc. Theoretical Aspects of Controlled Thernonuclear Research (1979).

18. C. E. Seyler, et a1., Comments Plasma Phys. Cont. Fusion, 4,21 (1978). 


\section{APPENDIX D--FIELD-REVERSED THETA-PINCH SCALING}

\section{R. E. Siemon}

\section{Introduction}

The model developed here is intended to be as simple as possible while displaying some of the presently understood features of a field reversed configuration generated by theta-pinch heating techniques. The plasma pressure profile is assumed to be uniform in a field free annulus as shown in Fig. D- 1 . While this "thin skin" approximation 1s clearly a poor approximation to the diffuse profiles observed experimentally, it provides considerable algebraic simplification. The density in the annulus corresponds to the maximum density
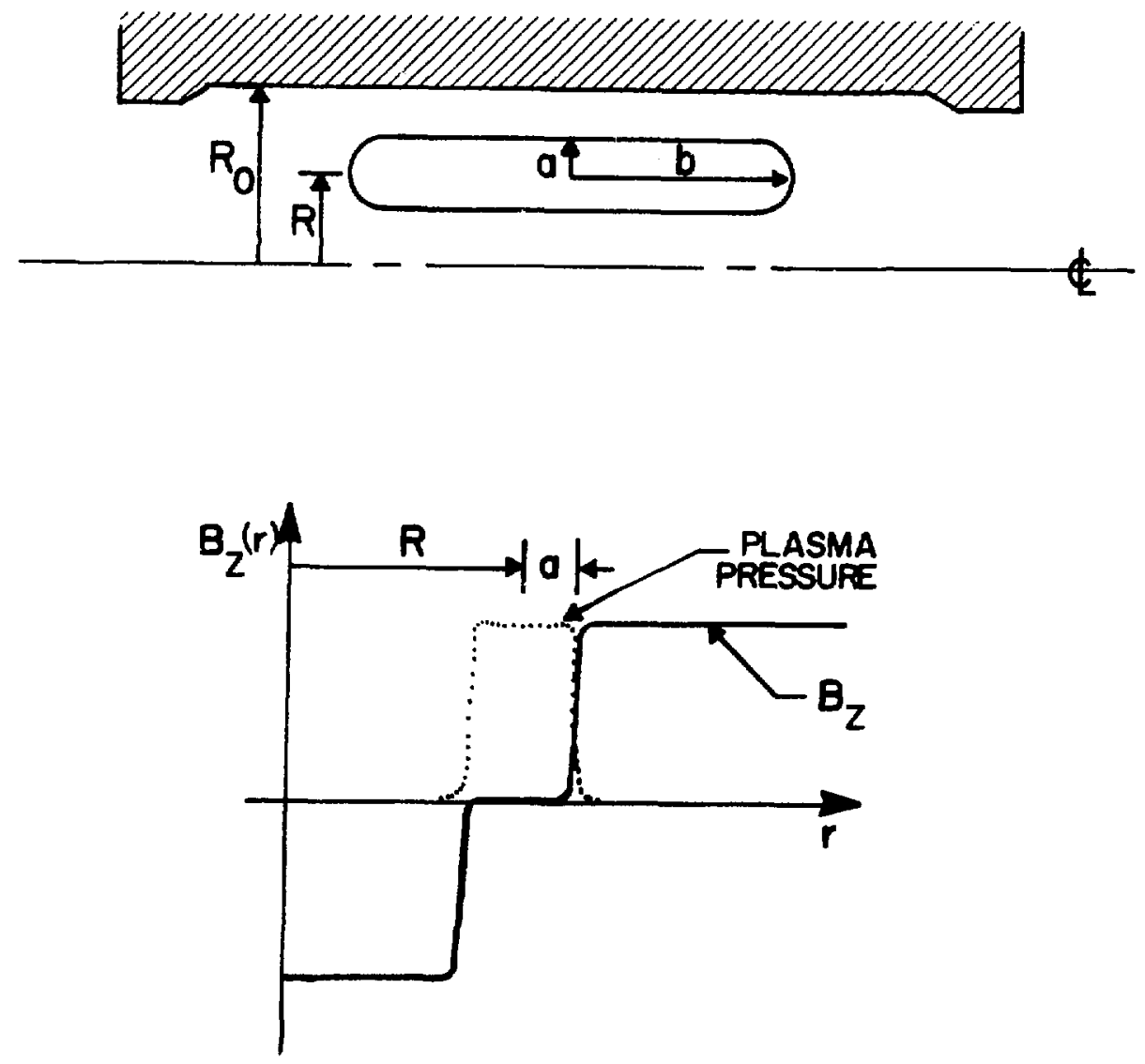

Fig. D-1.

Geometrical parameters for thin-skin plasma pressure approximation. 
of a diffuse profile $(\beta=1)$, and the radius a of the annulus corresponds to the characteristic width of the density in a diffuse profile. With some care in application of the results, it has generally been found that the "thin skin" model is a good starting point for developing scaling laws.

\section{Compression Factor $\mathrm{K}$}

From all experiments to date there appears to be a correlation between the amount of magnetic compression and the plasma lifetime. The compression factor $K$ is defined as follows

$$
K=\frac{B_{f}}{B_{i}}
$$

where $B_{f}$ is the final external magnetic fleld and $B_{i}$ is the initial bias field. The lifetimes from Table B-l of Appendix B are plotted in Fig. D-2 vs the factor $K$. Data are omitted for a few cases where the lifetime was stated to be limited by the magnetic field duration. A general tendency for increased lifetime with small $K$ is clear from the data, but the explanation for this correlation is uncertain. Wall stabilization may be involved since it is shown below that the plasma radius increases with reduced $k$. Equilibrium calculations indicate another effect. As $K$ increases, spacing between separatrix and plasma boundary at $\mathrm{R}+\mathrm{a}$ decreases as a result of two-dimensional equilibrium effects. It is not important for present purposes to explain the correlation. Rather, it is assumed that for scaling purposes the value of $\mathrm{K}$ must be kept comparable to the FRX experiments to insure stability. In this section and below the factors which influence $K$ are discussed.

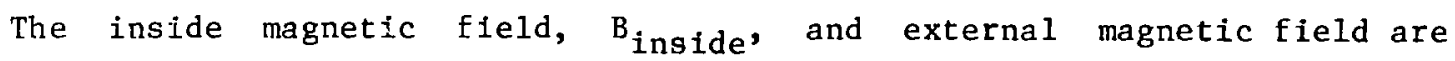
related through pressure balance

$$
\mathrm{n}_{\mathrm{f}} \mathrm{kT}=\frac{\mathrm{B}_{\mathrm{f}}^{2}}{8 \pi}=\frac{\mathrm{B}_{\text {inside }}}{8 \pi}
$$




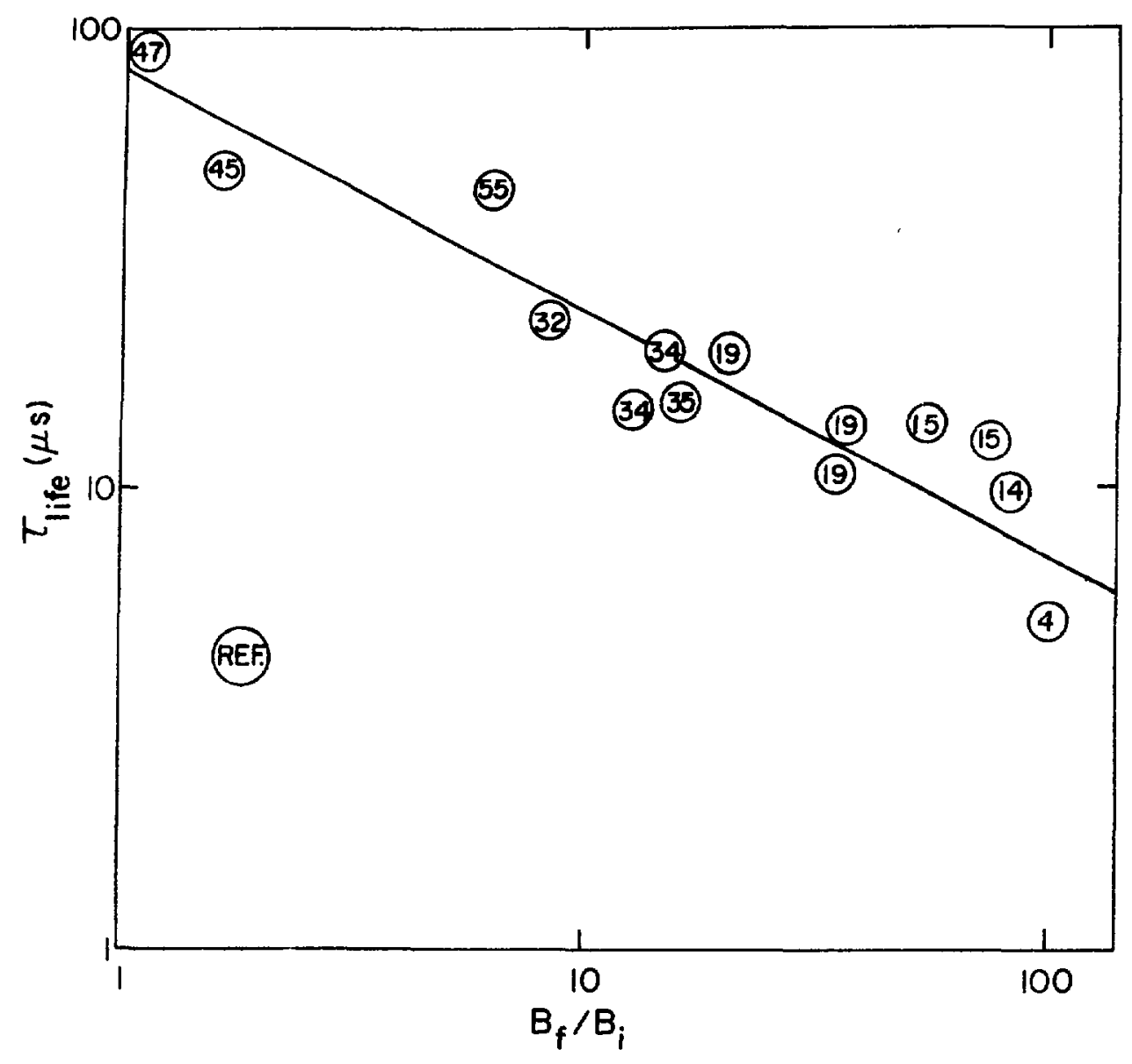

F1g. D-2.

Rough correlation of plasma lifetime with magnetic compression. Circled numbers are references in Appendix $B$.

where $B_{f}$ is the final external field, $n_{f}$ is the final maximum density (where $B$ $=0$ ) and $k T=k T_{e}+k T_{1}$. The plasma radius is determined by the quantity of trapped magnetic filux

$$
B_{f} R^{2}=k_{1} B_{i} R_{0}^{2}
$$

where $k_{1}$ is a constant less than unity representing the loss of initial flux during the turbulent implosion phase and $B_{1}$ is the initial bias magnetic field. In terms of $K$ the plasma radius $R$ is given by 


$$
\mathrm{R}=\left(\frac{\mathrm{k}_{1}}{\mathrm{~K}}\right)^{1 / 2} \mathrm{R}_{\mathrm{o}}
$$

In $F R X-B$ the measured values of $K(\simeq 5)$ and $R / R_{0}$ imply $k_{1} \approx 0.5$.

\section{Maximum Bias $B_{1}$}

The value of $\mathrm{K}$ is made small by making the initial bias as large as possible. However, there exists an upper limit, $B_{1}$, first discussed by Green and Newton. (See Appendix B.) The limit results from the initial phase of the implosion. The pre-ionized plasma with embedded flux finds itself unconfined near the wall as the external field begins to reverse. The maximum force of expansion occurs at the instant when the external field has zero magnitude, but an outward expansion continues until the applied magnetic field reverses direction and reaches the same magnitude as the initial bias. The flux lost as a result of the expansion requires detailed treatment of the resistivity of plasma interacting with the material wall. An approximate limiting value for the initial bias is obtained by requiring the field turn-on time to be much less than the Alfvén transit time,

$$
\begin{aligned}
& \frac{\mathrm{B}_{\mathbf{i}}}{\dot{\mathrm{B}}} \ll \frac{\mathrm{R}_{\mathrm{o}}}{\mathrm{V}_{\mathrm{A}}} \\
& \mathrm{V}_{\mathrm{A}}=\mathrm{B}_{\mathrm{i}} /\left(4 \pi \rho_{0}\right)^{1 / 2} .
\end{aligned}
$$

Thus

$$
B_{i}^{2} \ll R_{0} \dot{B}\left(4 \pi p_{0}\right)^{1 / 2}
$$

We define an upper limit on the bias to be 


$$
\mathrm{B}_{1}=\text { const } \times \mathrm{R}_{\mathrm{o}}^{1 / 2} \dot{\mathrm{B}}^{1 / 2} \rho_{\mathrm{O}}^{1 / 4}
$$

where the constant is chosen to give a limit in agreement with experiment. It is convenient to introduce the initial line density

$$
\mathrm{N}_{\mathrm{o}}=\pi \mathrm{R}_{\mathrm{o}}^{2} \mathrm{n}_{\mathrm{o}}
$$

in terms of which

$$
\mathrm{B}_{1}=\mathrm{k}_{\mathrm{B}} \dot{\mathrm{B}}^{1 / 2} \mathrm{~N}_{\mathrm{O}}^{1 / 4}
$$

Data from FRX-B indicate that for $B_{1}$ (gauss), $\dot{B}$ (gauss/sec) and $N_{0}\left(\mathrm{~cm}^{-1}\right.$ ) the value of $k_{B} \approx 1.4 \times 10^{-6}$. The maln purpose here is to develop the scaling rather than exact numbers. Green and Newton demonstrate that trapped flux (and thus R) varies as expected with increasing initial bias until an abrupt transition at a bias that scales as Eq. (5). Increasing the bias above $B_{1}$ does not increase the trapped flux because of the expanston process. The limit $B_{1}$ is set by the voltage $\left(\dot{B R}_{0}^{2}\right)$ and a weak dependence on $\mathrm{N}_{0}$. As explained later, variations in $N_{0}$ affect other quantities such as $a / \rho_{i}$.

\section{Optimum Compression, $\mathrm{K}_{1}$}

The choice $B_{1} \cong B_{1}$ corresponds to an optimum in the sense that $\mathrm{K}$ is as sma11 as possible with conventional theta-pinch heating. In considering the experimentally possible parameter varlations it is important not to increase $K$ since experiment indicates that the minimum $K$ produces the longest lifetime. The value of $k_{1}$ is defined as 


$$
\mathrm{K}_{1}=\frac{\mathrm{B}_{\mathrm{f}}}{\mathrm{B}_{1}}
$$

The scaling of the value of $\mathrm{K}_{1}$ with bank parameters

$$
V_{0}=\pi R_{0}^{2} \dot{B} / c
$$

and magnetic energy per unit length

$$
E_{m}=\left(B_{f}^{2} / 8 \pi\right) \pi R_{o}^{2}
$$

is

$$
\mathrm{K}_{1}=\frac{\mathrm{E}_{\mathrm{m}}^{1 / 2}}{\mathrm{v}_{0}^{1 / 2} \mathrm{~N}_{0}^{1 / 4}} \times \text { const }
$$

One feature to note is that the magnetic energy can not be increased alone, say by adding capacitors, without changing $K_{1}$. Experiments in the early 60's tended to use large $E_{m}$ and small voltage which is in a direction to increase $K_{1}$, reduce $R$, and limit the lifetime.

\section{Theta-pinch Heating}

Conventional scaling is

$$
k T=k_{3} \frac{\mathrm{B}_{\mathrm{f}} \dot{\mathrm{B}}^{1 / 2} \mathrm{R}_{\mathrm{o}}^{2}}{\mathrm{~N}_{\mathrm{o}}^{3 / 4}}
$$

where $k_{3}$ is adjusted to agree with the experiment. With temperature in $k e V, B_{f}$ 
(gauss), $\dot{B}$ (gauss/sec), $R_{0}(\mathrm{~cm}), N_{0}\left(\mathrm{~cm}^{-1}\right)$, the value of $k_{3}$ is about 42 for FRX-B and 64 for Scylla IV-P. The scaling is derived by starting with the snowplow equation

$$
\rho_{0} v_{r}^{2}=\frac{B^{2}}{8 \pi}
$$

During the implosion the B field vartes in time simply as $\dot{B} t$ where $\dot{B}$ is constant. Therefore

$$
V_{I}=\frac{\dot{B}}{\left(8 \pi \rho_{0}\right)^{1 / 2}} t
$$

The sheath reaches the axis in a time $t_{\text {imp }}$ which can be written in terms of an effective acceleration $a=\dot{B} /\left(8 \pi \rho_{0}\right)^{1 / 2}$

$$
t_{i m p}=\left(\frac{2 R_{o}}{a}\right)^{1 / 2} .
$$

The radial velocity increases linearly in time until the sheath reaches the axis. At that instant the directed energy is assumed to equilibrate. Following equilibration the increasing magnetic field provides further heating by adiabatic compression

$$
E^{T}=\frac{B_{f}}{B_{s}} \frac{1}{2} m_{i} v_{r}^{2}\left(t_{i m p}\right)
$$

ZEE $Z i \equiv Z d \bar{s}_{s}$ is the shock field at the end of the Implosion 


$$
\mathrm{B}_{\mathrm{s}}=\dot{\mathrm{B}} \mathrm{t}_{\mathrm{imp}}
$$

Combining Eqs. (9), (10), (11), and (12) gives the often used scaling law, Eq. (8) •

The model applies for initial bias fields of eufficiently small magnitude. The maximum bias is roughly estimated by requiring it to be small compared to the shock field $\mathrm{B}_{\mathbf{s}}$.

$$
B_{1} \ll B_{S}
$$

The value of $\mathrm{B}_{\mathrm{s}}$ obtained from $\mathrm{Eq} \cdot(9)$ is

$$
\mathrm{B}_{\mathrm{S}}=\dot{\mathrm{B}} \mathrm{t}_{1 \mathrm{mp}}=(32 \pi)^{1 / 4} \dot{\mathrm{B}^{1 / 2}} \mathrm{R}_{\mathrm{o}}^{1 / 2} \rho_{\mathrm{o}}^{1 / 4} .
$$

Comparison with Eq. (5) shows that the maximum bias consistent with heating scales identically to the maximum blas that is contained during the Initial transient while the field reverses.

One implication is that the optimum regime for stability, $\mathrm{K}=\mathrm{K}_{1}$, is inevitably associated with some compromise in heating efficiency and associated complexities in the heating process. Experimental data indicate that the compromise is not bad. The constant $k_{3}$ differs by less than a factor of two between Scylla IV-P with no bias and FRX-B with a bias.

Another implication concerns the use of multipole barrier fields to contain pre-lonized plasma as described by Kurtmullaev (see Appendix B). The above considerations show that increasing $B_{1}$ above $B_{1}$ necessarily corresponds to a reduction in heating efficiency. On the other hand, as Kurtmullaev points out, axial shock heating can still be employed. It is possible that Kurtmullaev's approach of barrier fields and axial shock heating offers advantages, and $1 t$ warrants further study. However, the present discussion is formulated under the assumption that conventional theta-pinch heating is being utilized. 


\section{Plasma Characteristic Thickness, a}

The remaining variable to determine is $a$, the half-width in the radial direction of the final plasma density profile. It is calculated by assuming that most of the initial particles in the tube are swept up by the implosion and contained during the subsequent axial contraction,

$$
2 \pi R(2 a) l_{f} n_{f}=k_{2} \pi\left(R_{o}^{2}-R^{2}\right) L_{o} n_{o}
$$

The constant $k_{2}$ is not yet well established in the FRX experiments but is probably close to unity as has been determined in some earlier experiments. In terms of previously defined variables

$$
a=\frac{2 k_{2} k_{3}}{k_{B} k_{1}^{l / 2}}\left(\frac{L_{o}}{\ell_{f}}\right)\left(1-\frac{k_{1}}{K}\right) \frac{K^{1 / 2}}{k_{1}} R_{o}
$$

It is assumed that $K$ might be less than $K_{1}$ since an initial bias less than $B_{1}$ is possible. The major dependence of $a$ is on $R_{0}$ if $K$ is chosen equal or close to $\mathrm{K}_{1}$.

A consequence of assuming that the heating is independent of the bias field is that the final density is also independent of bias field:

$$
\frac{n_{f}}{n_{0}}=\frac{k_{B}}{8 k_{3}^{\prime}} k_{1}
$$

where $\mathrm{k}_{3}=1.6 \times 10^{-9} \mathrm{k}_{3}$ to convert $\mathrm{keV}$ to ergs.

\section{Ratio a/p}

The ratio of radius, a, to ion gyro-radius, $\rho_{1}$, determines in many cases the finite-Larmor-radius (FLR) stabilization of short wavelength MHD instabilities. 
It is possible that present FRX experiments rely on such stabilization, and consequently a new experimental configuration must allow for operation with similar values of $a / \rho_{i}$. At the same time it is necessary to explore variations of the ratio since cross-field transport processes depend strongly on the same parameter. A reasonably large ratio $(>10)$ is probably necessary for meeting $\mathrm{n} \tau$ requirements in a fusion system.

The above scaling laws determine the ratio:

$$
\frac{a}{\rho_{i}}=\text { const } \times \frac{(a / R)^{1 / 2}\left(1-R^{2} / R_{0}^{2}\right)^{1 / 2}\left(L / \ell_{f}\right)^{1 / 2}}{\left(1+r_{T}\right)^{1 / 2}} N_{0}^{1 / 2}
$$

Here $r_{T}$ is the ratio of electron temperature to ion temperature which is necessarily introduced since the ion temperature determines the ion gyro radius. As long as $\mathrm{k} \approx \mathrm{K}_{1}$, the above relationship can be simplified

$$
\frac{a}{\rho_{i}} \cong \text { const } \times \mathrm{N}_{0}^{1 / 2}
$$

One concludes that $N_{0}$ is the most important variable in determining the ratio $a / \rho_{i}$. Voltage, discharge tube radius, final magnetic field, etc. are to first approximation of no consequence, although, unlike a conventional unbiased theta pinch, the result does depend weakly on other variables through such parameters as $\mathrm{a} / \mathrm{R}$ and $\mathrm{L} / \ell_{\mathrm{f}}$.

The selection of $\mathrm{N}_{0}$ is limited at the high end by loss of FLR stabilization and low temperatures (Eq. 8), and at the low end by anomalous resistance and poor implosion efficiency ("leaky piston"). The lower limit is derived by requiring that the commonly observed skin depth $c / \omega_{p 1}$ be small in comparison with the implosion distance $R_{0}$ :

$$
\frac{c}{\omega_{p i}} \leq R_{0}
$$


This Implies

$\mathrm{N}_{\mathrm{O}} \geq \operatorname{const}$

Most theta pinches work with $\mathrm{N}_{\mathrm{O}} \gtrsim 10^{16} \mathrm{~cm}^{-1}$ although the lower limit also depends on pre-ionization problems when the filling pressure is below a few mtorr.

\section{Heating with Fixed Compression}

The above scaling arguments can be summarized by expressing the temperature in terms of voltage, optimum compression factor, and filling pressure:

$$
\mathrm{kT}=\text { const } \times \frac{\mathrm{K}_{1} \mathrm{~V}_{\mathrm{o}}}{\mathrm{N}_{\mathrm{O}}^{1 / 2}} \text {. }
$$

It is clear from this equation that the desire for higher temperature with fixed $\mathrm{K}_{1}$ (for stability) and fixed $\mathrm{N}_{0}$ (to determine $a / \rho_{1}$ ) can only be met by going to higher voltage. One also sees that $a / \rho_{i}$ can be increased with fixed temperature by increasing $\mathrm{N}_{0}^{1 / 2}$ and $\mathrm{v}_{0}$ at the same time. 
APPENDIX E

Reprints of three recent papers on FRX-A and FRX-B experiments are attached.

1. Field Reversal Experiment (FRX)

Submitted to the Seventh International Conference on Plasma Physics and Controlled Nuclear Fusion Research, Innsbruck, Austria, Aug • 23, 1978.

2. Driven Mirror Studies on FRX-A

Submitted to Office of Fusion Energy, DOE, 1979.

3. Field Reversal Experiment

Submitted to the Ninth European Conference on Controlled Fusion and Plasma Physics, Culham, 1979. 
(Submitted to the Seventh International Conference on Plasma Physics and Controlled Nuclear Fusion Research, Innsbruck, Austria, Aug. 23, 1978.)

\author{
FIELD REVERSAL EXPERIMENTS (FRX) \\ R. K. Linford, W. T. Armstrong, D. A. Platts, E. G. Sherwood \\ University of California \\ Los Alamos Scientific Laboratory \\ Los Alamos, New Mexico 87545
}

I. INTRODUCTION

The reversed-field configuration (RFC) shown in Fig. I is topologically similar to the astron configuration, except that the toroidal current in the RFC is carried by thermalized plasma particles with $v_{d r i f t} v_{\text {thermal. RFC's }}$ have been produced in numerous theta pinch experiments [i-8jand recently by relativistic electron beams where the beam does not remain in the plasma $[9-10]$.

The early experiments, prior to the mid-60's, exhibited destruction of the RFC in less than 3is. The annihilation of the reversed field was not associated with any macroscopic distortion of the plasma shape, except for the disappearance of the central hole [2]. Observations included teariagmode-induced rings that nornaliy ccalesced before the annihilation [3], and the $m=2$ rotational instability that appeared afterwards $[1,2]$; however, the caise of the RFC destruction is not known.

More recent results have extended the l1fetime of the RFC up to

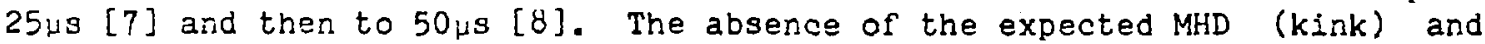
tearing instabilities has stimulated the hope that the RFC might be used to reduce end losses in linear systems. The RFC equilibrium and stability is being studied in two linear-theta-pinch facilities, FRX-A and FRX-B, in conjunction with several theoretical efforts.

\title{
II. EYPERIMENTAL SYSTEMS
}

Both FRX-A and FRX-B produce the RFC in a $20-\mathrm{cm} 1 . d$. quartz tube Inserted in a 25-cm i.d., one-turn, theta-pinch coli about I $m$ long. A $10 \%$ mirror field is produced by shaping the end of the coil. The majority of the detalled diagnostics have been done on FRX-A, which has a 7-kG peakfleld capability with a $3.2,3$ rise time. Simliar studies have been initiated on the more energetic FRX $-B(12 \mathrm{kG}$ and $1.8 \mathrm{\mu s}$ rise time).

The temporal behavior of the external B-field shown in Fig. 2 is produced by conventional crowbarred theta-pinch circuits operating in the "reversed bias" mode. The sequenoe begins with the slowly rising regative 
bias field, followed by the ringing theta-plnch preionization (PI), and then at $t=0$, the main field rises rapidly and is crowbarred with a 150-200 $\mathrm{ks}$ decay time. Comparison of the vacuum shot with the static 5 mTorr $\mathrm{D}_{2}$ fill shows the increased damping of the ringing PI as the plasma is formed. The bias fleld and PI plasma are allowed to diffuse before the -main field reverses the external field and implodes the plasma. The oppositely directed bias and main flelds reconnect near the ends of the system $[3,7]$ forming the RFC (see Fig. 1). The RFC excludes the open field lines from a substantial volume of the coil. This excluded flux effect results in $a$ lower coil inductance and $a$ higher coil current and external field shown in Fig. 2. When the RFC is destroyed (in this case, by the rotating $m=2$ mode), the field rapidly returns to the vacuum value. This effect has proved to be a reliable measure of the RFC lifetime. Fig. 2 represents typical data, but. not the longest lifetimes observed in the two machines.

other diagnostics are shown in Fig. 3. The spectrometer is used to estimate the electron temperature profiles by observing the ov $2781 \AA$, CIII $2297 \AA$, and CV $2271 \AA$ lines. The ion temperature and rotational velocity is inferred from the polychromator measurement of the Doppler broadening and shift of the CV $2271 \AA$ line. In addition to the end-on interferometer shown in Fig. 3, a feedback stabilized interferometer was used to measure the line density through the plasma diameter at various axial positions. A pressure probe [11] has also been used to measure the spatial and temporal behavior of the plasma escaping out of the end of the system.

\section{EXPERIMENTAL RESULTS}

The macroscopic behavior of the plasma is indicated by the side-on streak and end-on framing pictures in Fig. 4. Five phases of the plasma behavior can be seen during the usual "clean" mode of operation (Fig. 4(b)): (1) PI $(t<0)$, (2) implosion, field reconnection, and axial contraction $(0<t<5 \mu s)$, (3) equilibrium ( $5<t<15 u s)$, (4) onset and growth of the rotational $\mathrm{m}=2$ instability $(15<t<19 \mu \mathrm{s})$, and (5) the discuption of the RFC and loss of confinement $(19<t<23 \mu s)$. This behavior is very similar to that ooserved by Eberhagen and Grossmann [7].

An abrupt switch from clean to "dirty" mode operation (Fig. 4(a)) is produced by increasing the fill pressure, lowering the plasma purity, or lowering the power input to the plasma. This behavior suggests that the dirty mode is caused by failure to burn through a radiation barrier. The sudden destruction of the RFC before the onset of the rotational instability (Fig. 4(a)) characterizes the dirty mode and was observed in the behavior of the early experiments; however, the mechanism of the early RFC destruction is not understood. The dirty mode plasma contains CIII throughout the plasma life, indicating an electron temperature $\mathrm{T} \mathrm{e} \leqslant 20 \mathrm{eV}$. During the clean mode, $O V$ burns through during the implosion and $\mathrm{CV}$ exists during equilibrium and instability phases, indicating that $\mathrm{T}_{\mathrm{e}} \sim 100 \mathrm{eV}$ (see Fig. 5). The remainder of the paper considers only clean mode behavior.

The axial profiles of transverse line density shown in Fig. 6 confirm the plasma length of about $35 \mathrm{~cm}$ indicated by the streak photographs during the equilibrium phase. The axial profiles are also confirmed by $C V$ radiation measurements. The hollow radial profile indicated by the framing pictures is also confirmed by $\mathrm{CV}$ radiation and end-on interferonetry. 
However, accurate measurements of the radial density profile are hindered by cold plasma outside the region of the implosion coll.

Ion temperatures are inferred from the Doppler broadening of the CV $2271 \AA$ Iine, which does not suffer from signicant Stark effects in the density range of interest. Depending on the strength of the implosion field, $\mathrm{T}_{i}$ varies between 150 and $350 \mathrm{ev}$. For the densities of interest, the equilibration time between the $\mathrm{CV}$ and deuterium is less than 5us. The ion temperature is independent of radius and appears to decrease by about $20 \%$ during the $14 \mu \mathrm{s}$ of the equilibrium and instability phases. Although this decrease is about the same as the uncertainty of the measurement, it is also the amount that is consistent with classical equilibration between electrons and ions, assuming $T_{e}$ is fixed.

During the equilibrium and instability phases, the temporal behavior of the plasma size, density (Fig. 6), electron temperature (Fig. 5), and ion temperature indicate energy and particle confinement times that exceed $40 \mathrm{us}$, which is more than 10 times the expected confinement in an open-field system. Furthermore when the RFC is disrupted, the observed particle loss rates are consistent with the expected 3 to $4 \mathrm{HS}$, and the drop in CV (Fig. 5) is consistent with the slightly faster electron thermal losses expected $[12,13]$ along open-field lines.

These results indicate that the enhanced confinement is due to closed-field lines, but the reversed field has also been measured directly. A magnetic field probe was inserted along the axis of the system that measured reversed fields of the order of $45 \%$ of the external fields. However, this internal probe shortened the Iifetime of the RFC and was removed.

Pressure prote measurements of the plasma escaping from the end of tine coil region confirmed and clarified several other measurements. A short, small burst of pressure was measured at all radii, which apparently corresponds to plasma lost at the time of implosion before the field line closure. All subsequent plasma loss occurred in a circular jet of plasma along the system axis less than $4 \mathrm{~cm}$ in diameter at the mirror peak and expanding along a vacuum flux tube. Time of flight measurements of this jet indicate that the majority of plasma loss occurs in two equally energetic bursts of similar mass that originate during (1) the axial contraction and (2) just following the RFC disruption. Between these two bursts (during the equilibrium and instability phases) the pressure from the escaping plasma corresponds to a characteristic loss time of 50 to $80 \mathrm{us}$. The first pressure burst corresponds to the loss of $1 / 2$ the initial fill during the axial contraction. This measurement is consistent with the measured inventory after the axial contraction. The magnitude of the two pressure peaks also agrees with a plasma pressure equal to the confinini field pressure $(3=1)$. In addition, assuming an appropriate rigid rotor density profile, the measured density, temperatures, and $B$ field are consistent with pressure balatce. The cause of the loss of plasma during the contraction phase is not yet understood. 
Ion rotational velocity is determined by the Doppler shift of the CV $2271 \AA$ line along a varlety of chords through the plasma. The tempcral behavior of the observed rigid-rotor rotation is shown in Fig. 7. The onset of the rotational instability always occurs at the same normalized value of rotation.

Several minor modifications of the system have been made in an attempt to limit the rotation or generally to extend the life of the plasma. Since Kurtmuliaev and co-workers [7] did not see the $m=2$ mode, hexapole fields were added and both first- and second-half-cycle operation were used in an effort to duplicate their results. No significant change in the maximum attainable lifetimes was observed.

Cuartz end plugs were inserted in an effort to control the radial electric field and the associated rotation [14]. The effects were generally detrimental, but the results were clouded by possible interference of the plugs with the PI process. Experimentally, the plasma stability is very sensitive to the PI conditions.

The passive mirrors were removed from the end of the main coil in FRX-A and separately powered mirror coils were added. With a 1 us quarter period risetime and a mirror ratio $\sim 2$, the uncrowbarred mirrors resulted in $R F C$ lifecimes up to $45 \mathrm{us}$. The plasma parameters under these conditions are being measured. The mirror power supply will soon be crowbarred to provide additional axial implosion energy, similar to the Kurtmullaev experiment. A possible explanation for the improved lifetime is that more rapid closure of the field lines confines a larger fraction of the plasma, and hence, greater inertia opposes the accelerating torque.

The higher energy FRX-B can produce up to $40_{\mu}$ SFC lifetimes with the original passive mirrors.

IV. DISCUSSION

The radial equilibrium profile of the observed RFC is probabiy similar to an infinitely long system because the length $\sim 35 \mathrm{~cm}$ is long compared to Its radial dimensions $\leqslant 6 \mathrm{~cm}$. Viscosity will cause the radial equilibrium to approach a rigid-rotor profile. Although existing measurements such as ion rotation are all compatibie with the rigid-rotor model, accurate radial density and $B-f i e l d$ profiles are needed to determine the actual radial equilibrium. The axial equilibrium behavior of the plasma is similar to the results of belt-pinch equilibrium calculations $[15,16]$. The plasma in both FRX systems contracts to about $1 / 3$ of the coil length and oscillates slightly with the crowbarred field modulation (Fig. 2) with very little change in radial dimensions. Several attempts are being made [17] to calculate the equilibrium shape with appropriate FRX boundary conditions.

The observed confinement time during the equilibrium phase of about 40 to $80 \mathrm{ws}$ is longer than predicted for Bonm diffusion, but is substantialiy shorter than the $300 \mu \mathrm{s}$ classical time, assuming the estimate of $\mathrm{T}_{\mathrm{e}}=100 \mathrm{eV}$ is accurate. These shorter confinement times might be caused by microinstability-enhanced diffusion $[18,19]$ or by ion-ion diffusion. This likeparticle diffusion can dominate electron-ion diffusion for small values of $a / \rho_{i}$ where a is the minor radius of the plasma and $\rho_{i}$ is the ion gyro radius in the external field [20]. For the observed values of $2<a / 0_{i}<3$, both 
sources of anomalous diffusion are viable. The diagnostic studies of the recently observed longer-lived plasmas should increase the accuracy of the measured particle and energy confinement time.

The study of the stability properties of the FRX-produced plasmas is dominated by the rotational $m=2$ mode. The rotational modes for a standard theta plnch have been theoretically determined by Freidberg and Pearlstein [21]. The theory predicts a stable range of normalized rotation $0<-2 / \Omega *<1$ for a rigid-rotor profile. Freidberg modified the model to handle the rigid-rotor RFC. He made a simple, but not unique, analytic continuation in the vicinity of the singular point at $B=0$. The result predicted 
the critical rotation of $-\Omega / \delta \sim 1.6$ for the $m=2$ mode. Because the finite Larmor radius (FLR) expansion used in the code breaks down near $B=0$, the validity of the result was suspect. However, the results were verified $\left(-\Omega / \Omega^{*} \sim 1.5\right)$ by using a Vlasov fluid code [22] that correctly calculates the orbits near $\mathrm{B}=0$.

The experimental and theoretical results qualitatively agree that a stable range of rotation does exist. However, the theoretically predicted value of the critical rotation is four times higher than experimentally observed. The error bars in Fig. 7 do not include the uncertainty in determining $\Omega$ from measured quantities, but it is certainly accurate to within a factor of 2. Other possibilities exist to explain the error, such as that the RFC may not have the assumed rigid-rotor equilibrium, or tine $C V$ ions may not be rotating at the same velocity as the deuterium, even though calculations indicate they should.

The apparent lowering of the rotational threshold for instability may arise from a coupling between the rotational mode and the MHD kink mode. Because of the geometry, the fastest growing kink mode probably results in the same $m=2$ distortion. Even though geometry and FLR effects may reduce the growth rate of the kink so that it is not observed in a non-rotational fom during the plasma lifetime, the free energy in the field that drives the kink may lower the threshold of the rotational mode. Since both rotational models are one dimensional, they do not contain tinis possible driving force. Freidberg is examining the possibility of adding a gravitational term to simulate this effect. In addition, a 3-dimensional MHD code [23] is being used to study the effects of geometry and rotation on the kink mode $[24]$.

The macroscopic tearing of the plasma into distinct rings has never been observed in the FRX systems. In spite of recent advances in the understanding of tearing modes [25,26], a two-dimensional model may have to be developed to accurately determine the stability of finite length layers as produced in the FRX systems. But even if long layers tend to tear into distinct rings, the result may not be detrimental to fusion concepts [27].

The continuing focus of research is to understand and control the $m=2$ rotational instability. Detailed experimental studies of the dependence of this mode on temperature, geometry, and RFC formation process are being pursued. Advanced theoretical models including geonetry and kinetic affects are under development.

\section{ACKNOWLEDGEMENT}

The assistance of Dr. C. J. Buchenauer in the development of the UV polychromator is gratefully acknowledged. In addition, the expert technical services of G. L. Stelzer and G. M. Sandoval are greatly appreciated. 


\section{REFERENCES}

[1] ROSTOKER, N., KOLB, A. C., Phys. Rev. 124 (1961) 965.

[2] BODIN, H. A. B., NEWTON, A. A., Phys, Fluids 6 (1963) 1338.

[3] BODIN, H. A. B., Nucl. Fusion 3 (1963) 215.

[4] LITTLE, E: M., CUINN, W. E., Phys. Fluids 6 (1963) 875.

[5] ALIDIERES, M., AYMAR, K., JOURDAN, P., KOCCHLIN, F., SAMAIN, A., Plasma Phys. 10 (1968) 841.

[6] KALEK, A., KONEN, L., NOLL, P., SIGUTA, K., WAELBROEK, F., WATANABE, K., WITULSKI, H., "Limitation of the Confinement of Plasma in a Liner Theta Pinch with Trapped Reverse Magnetic Field," Conf. Plams Phys, and Contr. Nucl. Fusion, Novosibirsk, Vol. II (1968) 581.

[7] ERERHAGEN, A., GROSSMANN, W., Z. Phys, 248 (1971) 130.

[8] ES'KOV, A. G., et al. "Plasma Confinement in a Pulsed System With a Compact Toroidal Configuration." (Proc. Seventh European Conf. Controlled Fusion and Plasma Physics 1975) 1 Lausanne (1975) 55.

[9] HAMMAR, D. A., ROBSON, A. E., GERBER, K. A., SETHIAN, J. D., Physical Lett. $60 A$ (1977) 31.

[10] ROBERSON, C. W., Bull. Am. Phys. SOc. 22 (1977) 1168.

[11] YORK, T. M., Rev. Sci. Instrum. 41 (1970) 519.

[12] CHU, T. K., JOHNSON, L. C., Phys, Fluids 20 (1977) 1684.

[13] GARY, S. P., SGRO, A. G., DESILVA, A. W., submitted to Phys. Fluids.

[14] COMMISSO, R. J., EKDAHL, C. A., FREESE, K. B., MCKENNA, K. F., CUINN, W. E., Phys, Rev. Lett. 39 (1977) 137.

[15] KADISH, A., SiEVENS, D. C., Nucl. Fusion 14 (1974) 821.

[16] BAKER, D. A., MANN, L. H., "Toroidal Belt Pinch Equilibria," Los Alamo3 Scientific Laboratory internal report LA-UR-73-104 (19?3).

[17] MANN, L. W. (LASL), ANDERSON, D. V. (LLL), GROSSMANN (NYU), persOHal communication.

[18] DAVIDSON, R. C., KRALL, N. A., Nucl. Fusion 17 (1977) 1313.

[19] HAMASAKI, S., KRALL, N. A., Bull. Am. Phys. Soc. 22 (1972) 1136.

[20] TUSZEWSKI, M., LICHTENBERG, A. J., Phys. Fluids 20 (1977) 1263.

[21] FREIDBERG, J. P., PEARLSTEIN, L. D., to be published in Phys. Fllids.

[22] SEYLER, C. E., to be published.

[23] BRACKBILL, J. U., Meth. Comp. Phys., 16 (1976) 1.

[24] ANDERSON, D. V., personal corimunication.

[25] FINN, J. M., KAW, P. K., Phys. Fluids 20 (1977) 72.

[26] DRAKE, J. F., LEE, Y. C., Phys. Fluids 20 (1977) 1341.

[27] GROSSMANN, W., SEYLER, C. E., STEINHAUER, L., Bull. Am. Phys. SOc. 22 (1977) 1189. 


\section{FIGURE CAPTIONS}

Fig. 1. Reversed-field configuration.

Fig. 2. Comparison of the external $B$ field measured in FRX-A and FRX-B between the quartz tube and the coil at the axial midplane. Traces are shown for both vacuum and a static 5 mTarr $D_{2}$ fill pressure.

Fig. 3. Schematic arrangement for some of the diagnostics on FRX-A.

Fig. 4. Side-on streak and end-on framing pictures for the (a) dirty mode and (b) clean mode of operation. The framing rate is $1.7 \mu \mathrm{s} / \mathrm{frame}$, with the first frame at $t=3 \mu s$. The thin, outer luminous ring in the framing pictures corresponds to the $20-\mathrm{cm}$ diameter quartz tube.

Fig. 5. Oscilloscope traces comparing the temporal behavior of the (a) CIII $2297 \AA$ radiation, (b) $\mathrm{CV} 2271 \AA$ radiation, and (c) the external magnetic field for clean mode operation. The intensities of the two carbon lines are plotted on the same scale.

Fig. 6. The line density through the RFC diameter as a function of axial position for three times during the equilibrium phase. The connecting lines are added for visual clarity.

Fig. 7. The temporal history of the CV rotational velocity normalized to the deuterium diamagnetic drift velocity. 


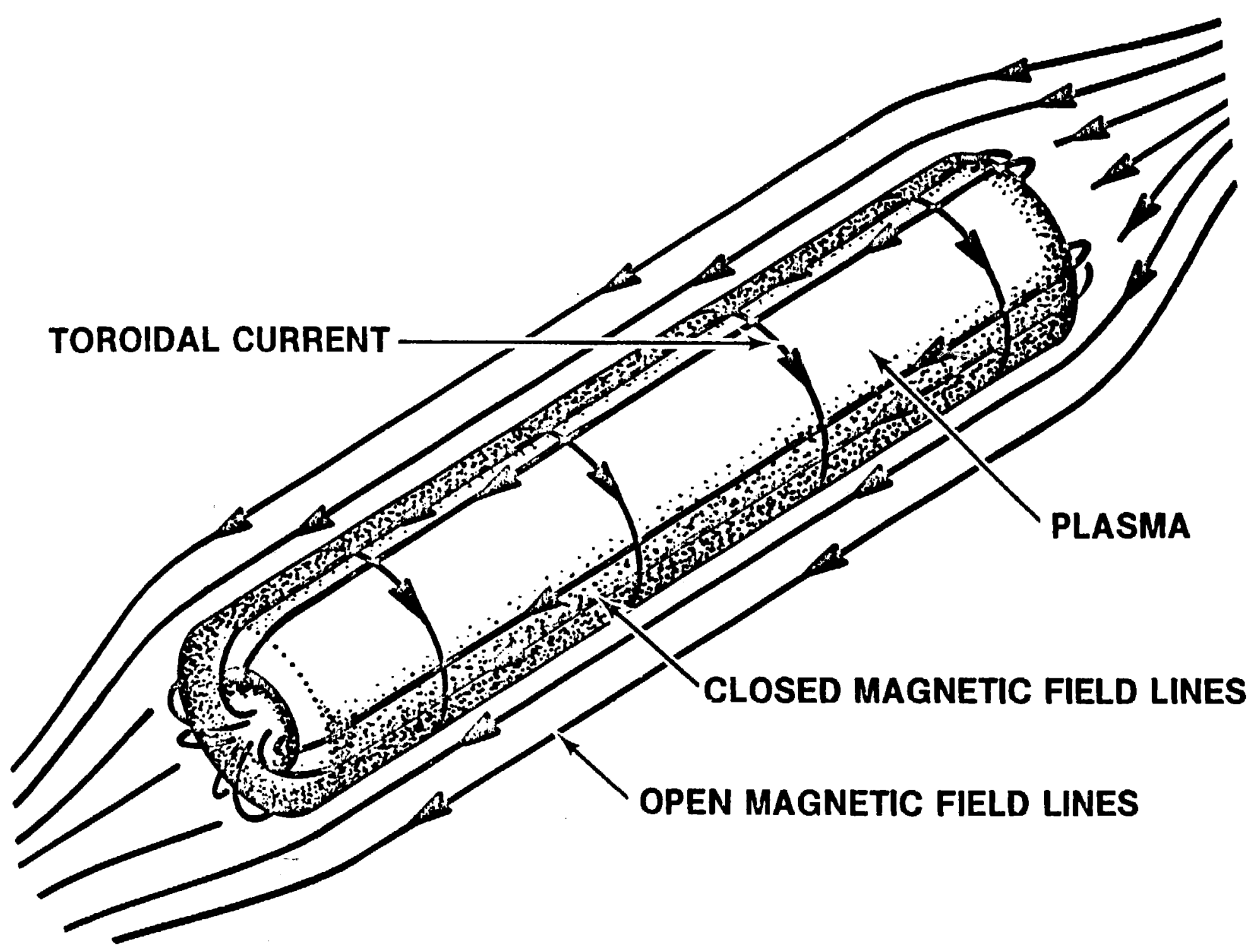

Fig, 1. Reversed-field configuration. 

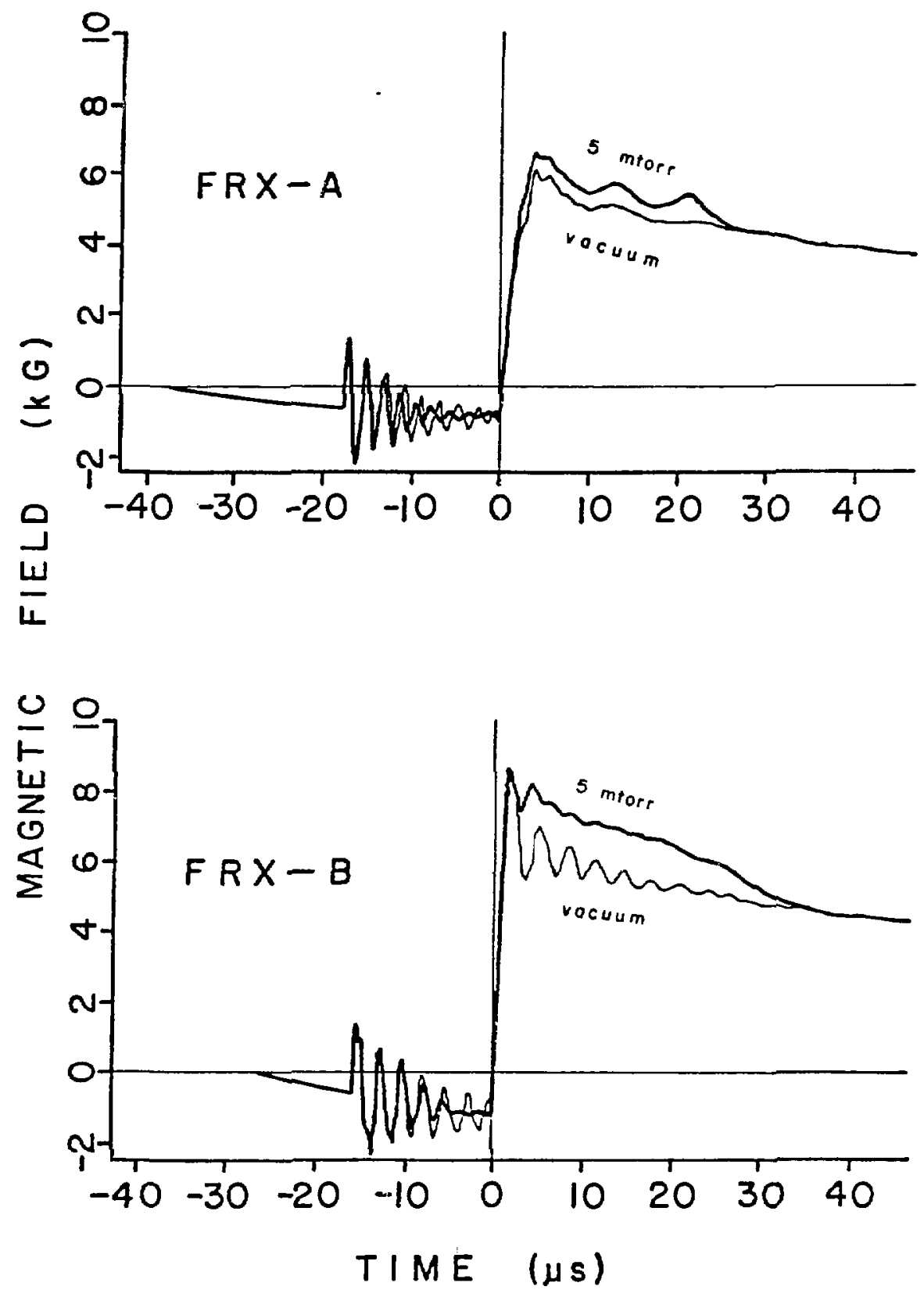

Fig. 2, Comparison of the external B field measured in FRX-A and FRX-B between the quartz tube and the coll at the axlal midplane. Traces are shown for both vacuum and a static 5 mtorr $D_{2}$ fill pressure. 


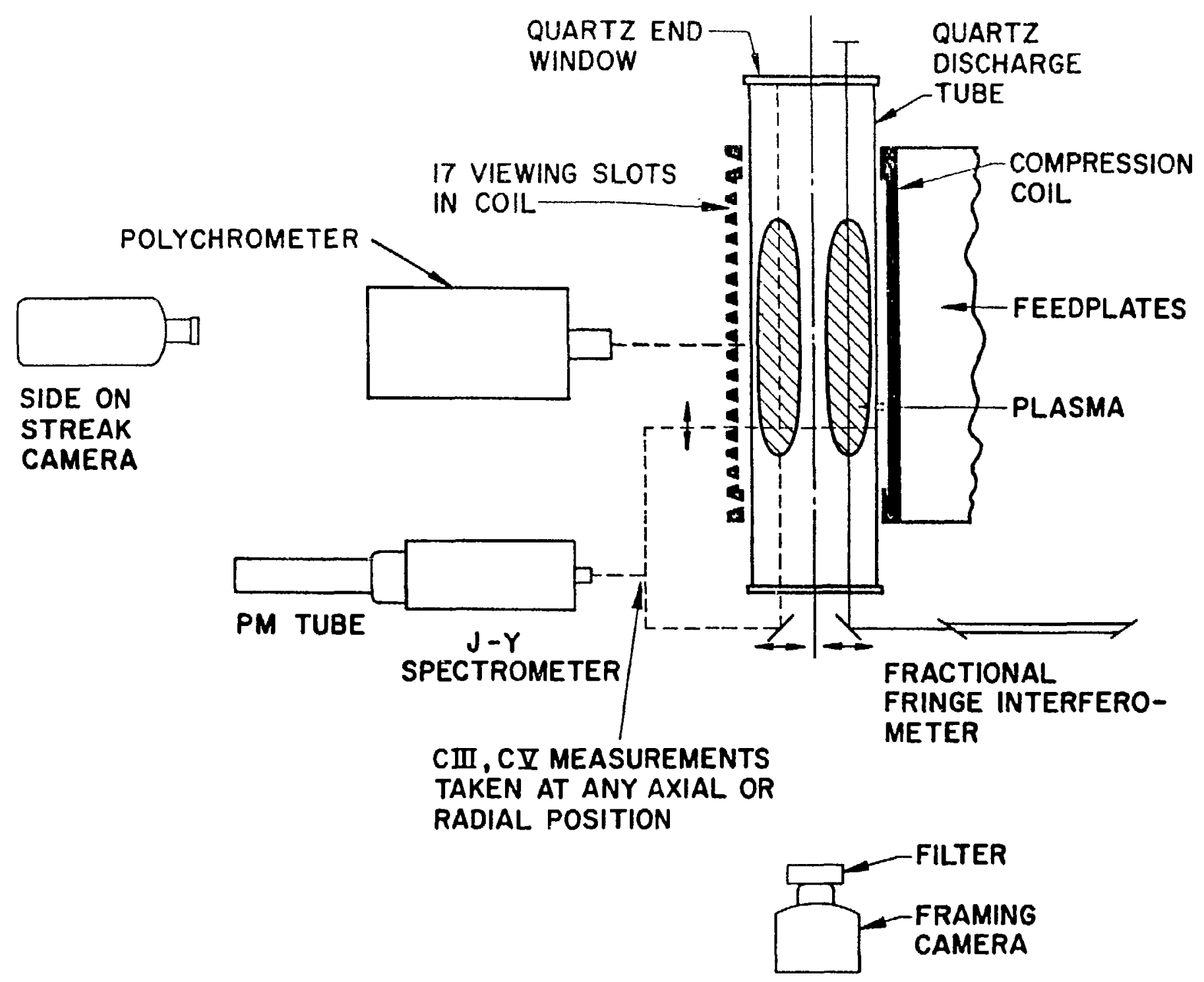

Fig. 3. Schematic arrangement for some of the diagnostics on FRX-A. 

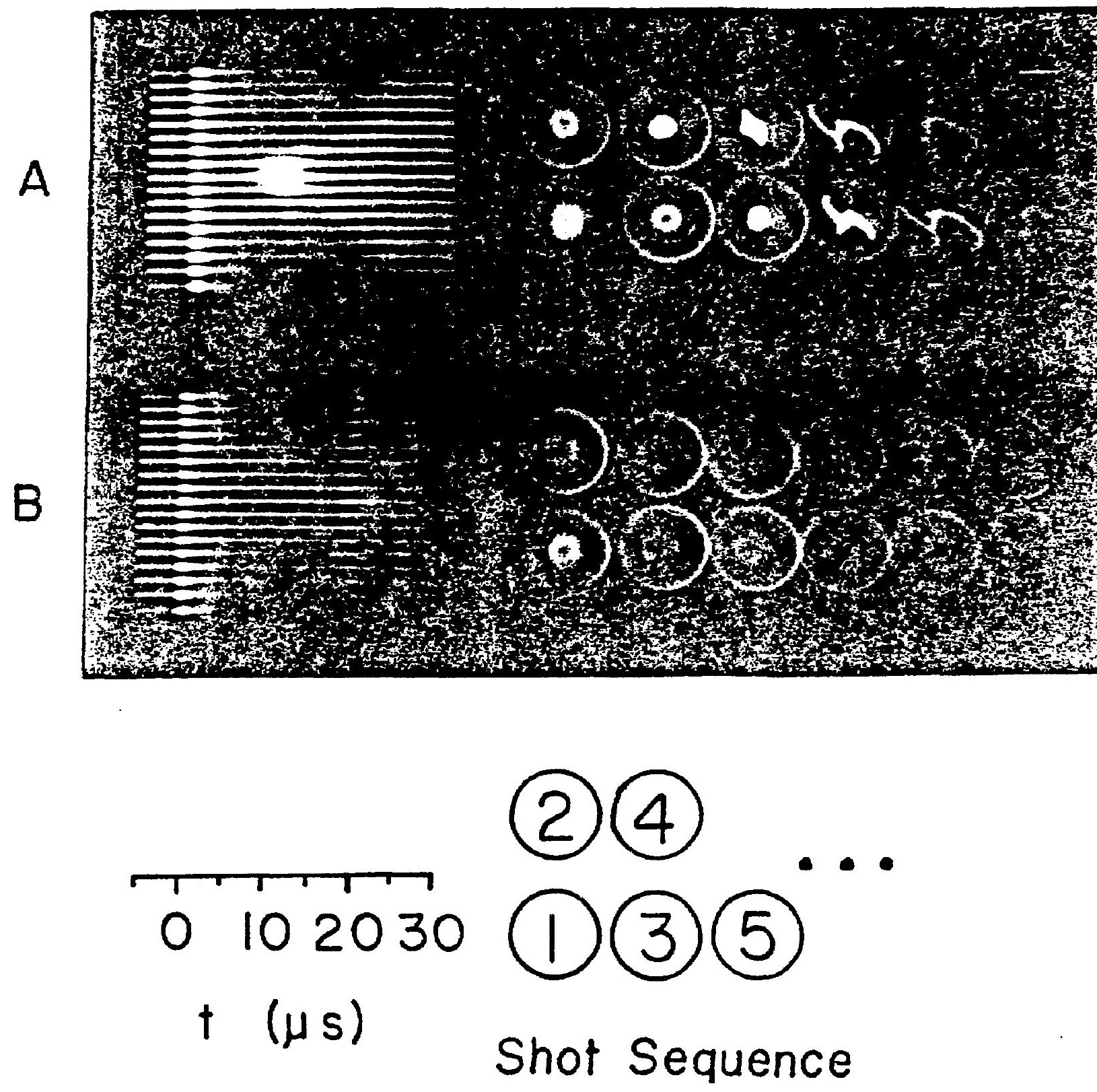

Fig, 4, Side-on streak and end-on framing pictures for the (A) dirty mode and (B) clean mode of operation. The framing rate is $1.7 \mathrm{Ls} / \mathrm{frame}$, with the first frame at $t=3 \mu \mathrm{s}$. The thin, outer luminous ring in the framing pictures corresponds to the $20-\mathrm{cm}$ diameter quartz tube. 


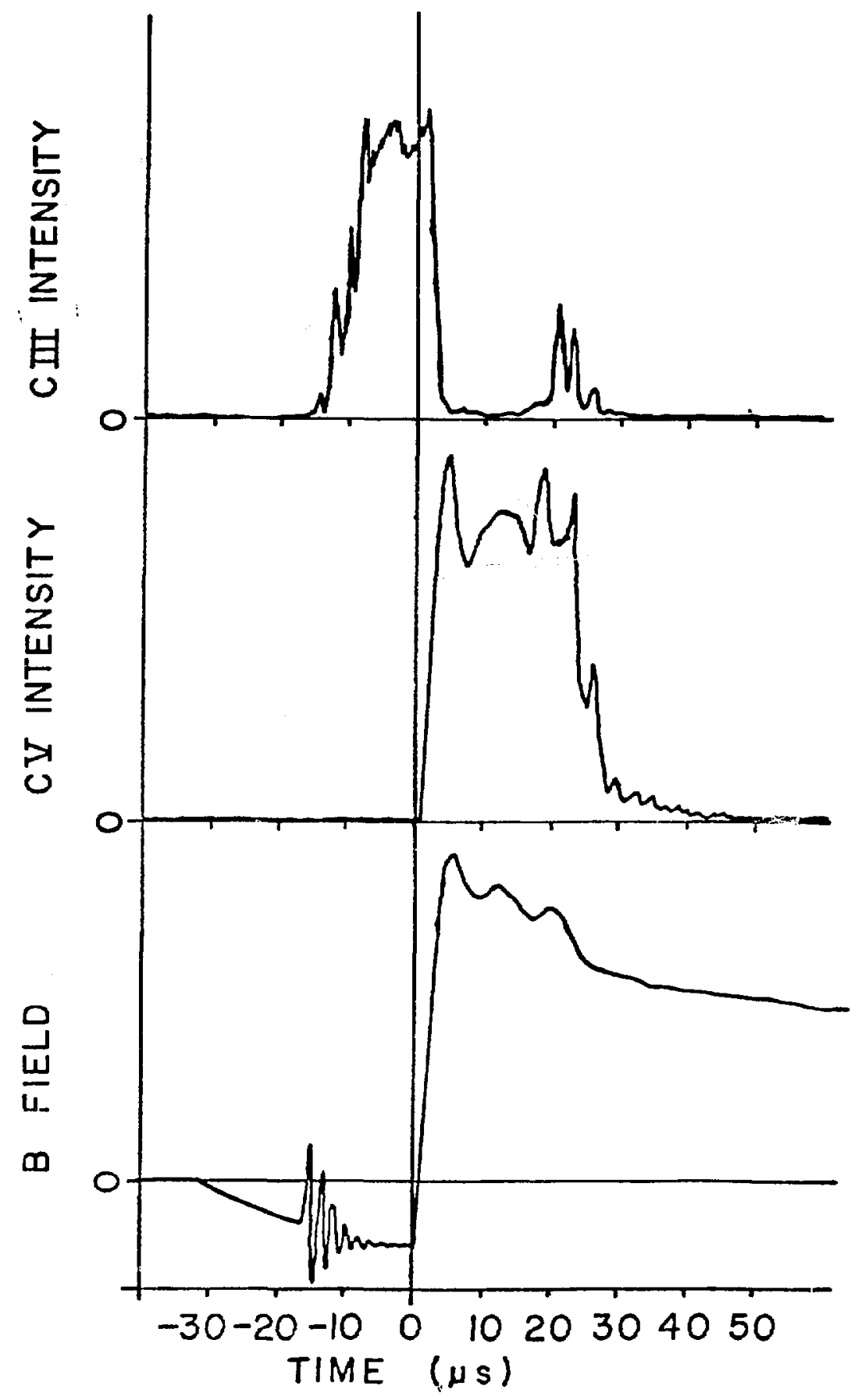

Fig, 5, Oscilloscope traces comparing the temporal behavior of the (A) CIII $2297 \AA$ radiation, (B) CV $2217 \AA$ radiation, and (C) the external magnetic field for clean mode operation. The intensities of the two carbon lines are plotted on the same scale, 


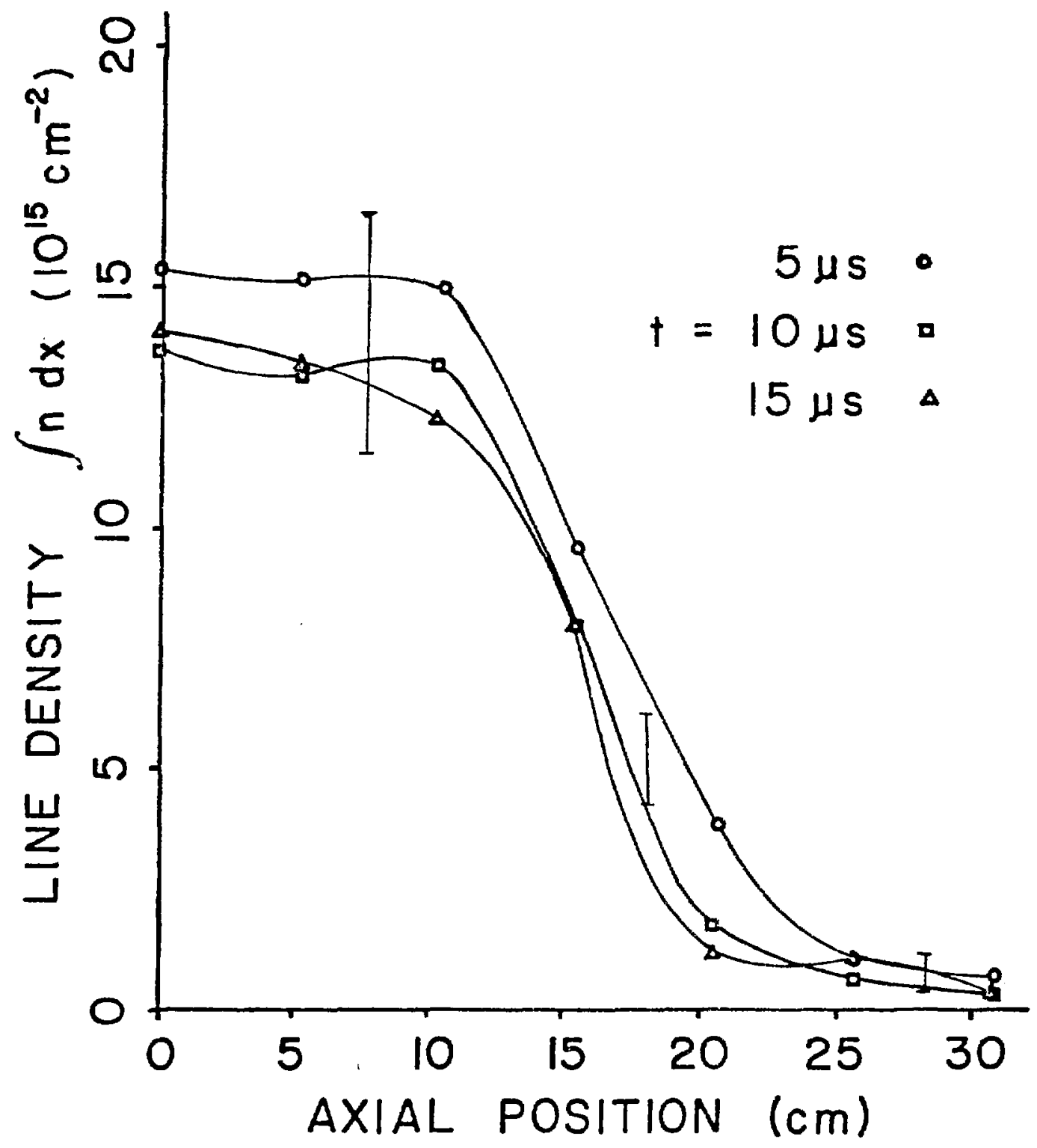

Fig: 6, The line density through the RFC diameter as a function of axial position for three times during the equilibrium phase. The connecting lines are added for visual clarity. 


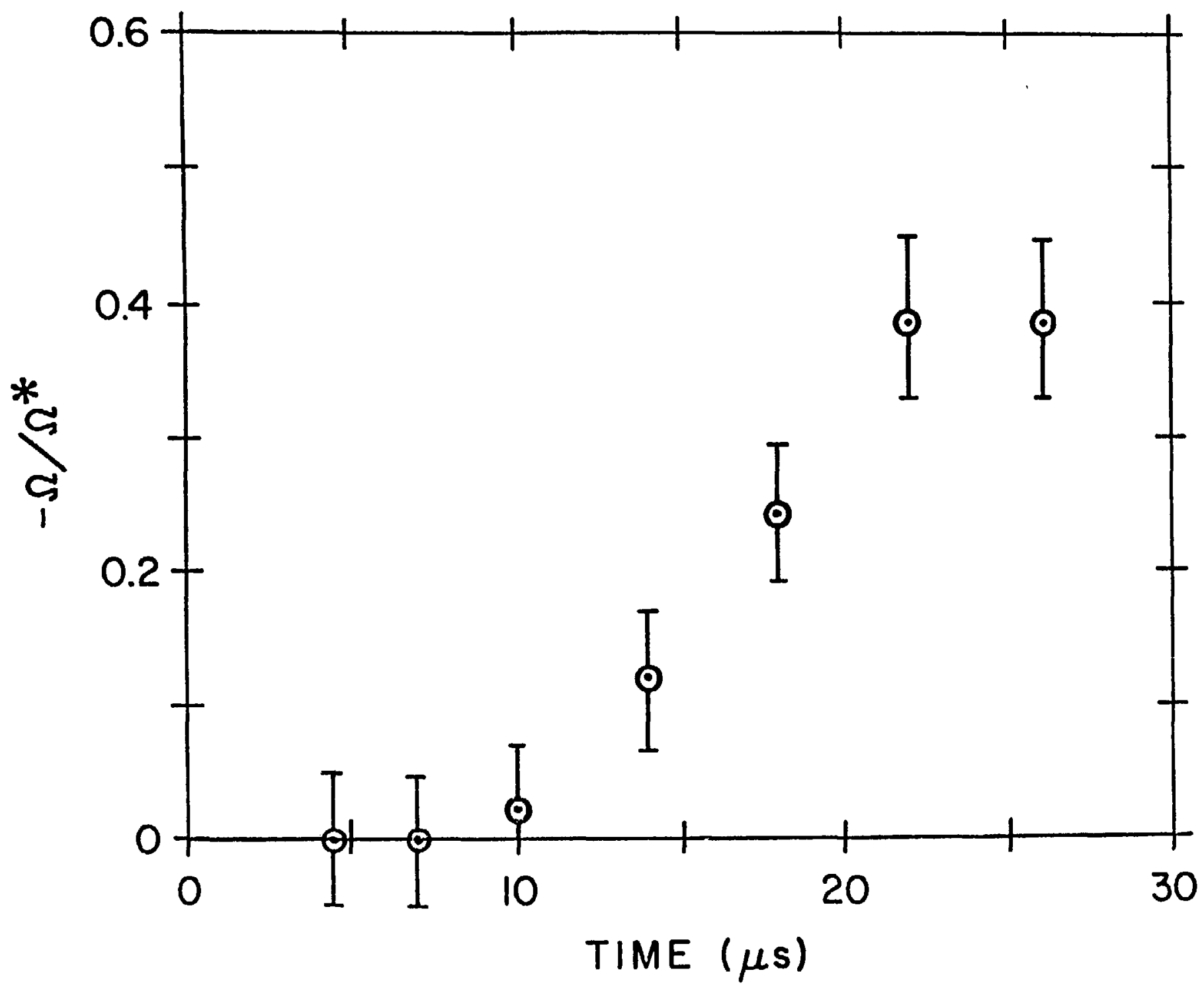

Fig, 7. The temporal history of the $\mathrm{CV}$ rotational velocity normalized to the deuterium diamagnetic drift velocity. 
(Submitted to the Office of Fusion Energy, Department of Energy, 1979.)

\author{
DRIVEN MIRROR STUDIES ON FRX-A
}

W. T. Armstrong, R. K. Linford, J. Lipson, D. A. Platts, E. G. Sherwood

\title{
A. INTRODUCTION
}

The passive mirrors shown in Fig. 1(a), which are normally used on both FRX-A and FRX-B, were removed from FRX-A and the independently driven mirrors shown in Fig. $l(b)$ were installed. When the operating parameters were optimized with the new driven mirrors, the maximum obtainable plasma lifetimes were increased by more than 30 percent. These previously reported results motivated additional studies directed at understanding the effect of driven mirrors on plasma lifetime. This report briefly summarizes these studies, which include measurements of various plasma parameters both with and without energizing the driven mirror coils, and compares these results with data previously obtained with the passive mirrors. In addition, the report describes the formation and translation of the plasma out of the end of the system when only one mirror coll is energized.

\section{B. SYMMETRICAL DRIVEN MIRROR EXPERIMENTS}

The lifetime of the plasma was maximized by varying all of the avallable parameters except for the maln magnetic field. This field was kept at about $5.5 \mathrm{kG}$ as measured $10 \mu \mathrm{s}$ after the implosion. The optimum pressure was found to be 7 mtorr both with and without the mirrors energized in contrast to the 5 mtorr value obtained with the passive mirrors. The optimum timing of the driven mirrors coincided with the inftiation of main bank, and an optlmum magnitude for the mirror field was also determined. The plasma behavior was essentially independent of whether the mirror field was crowbarred or not.

After these optimum conditions were determined, the discharge tube was brought up to atmospheric pressure and the B-field on axis was measured as a function of axial position. Flg. 2 shows this axial profile for several times during the initial rise of the field when the mirror coils were energized. Fig. 3 compares the theoretically determined field for the passive mirrors, and the measured field, for 

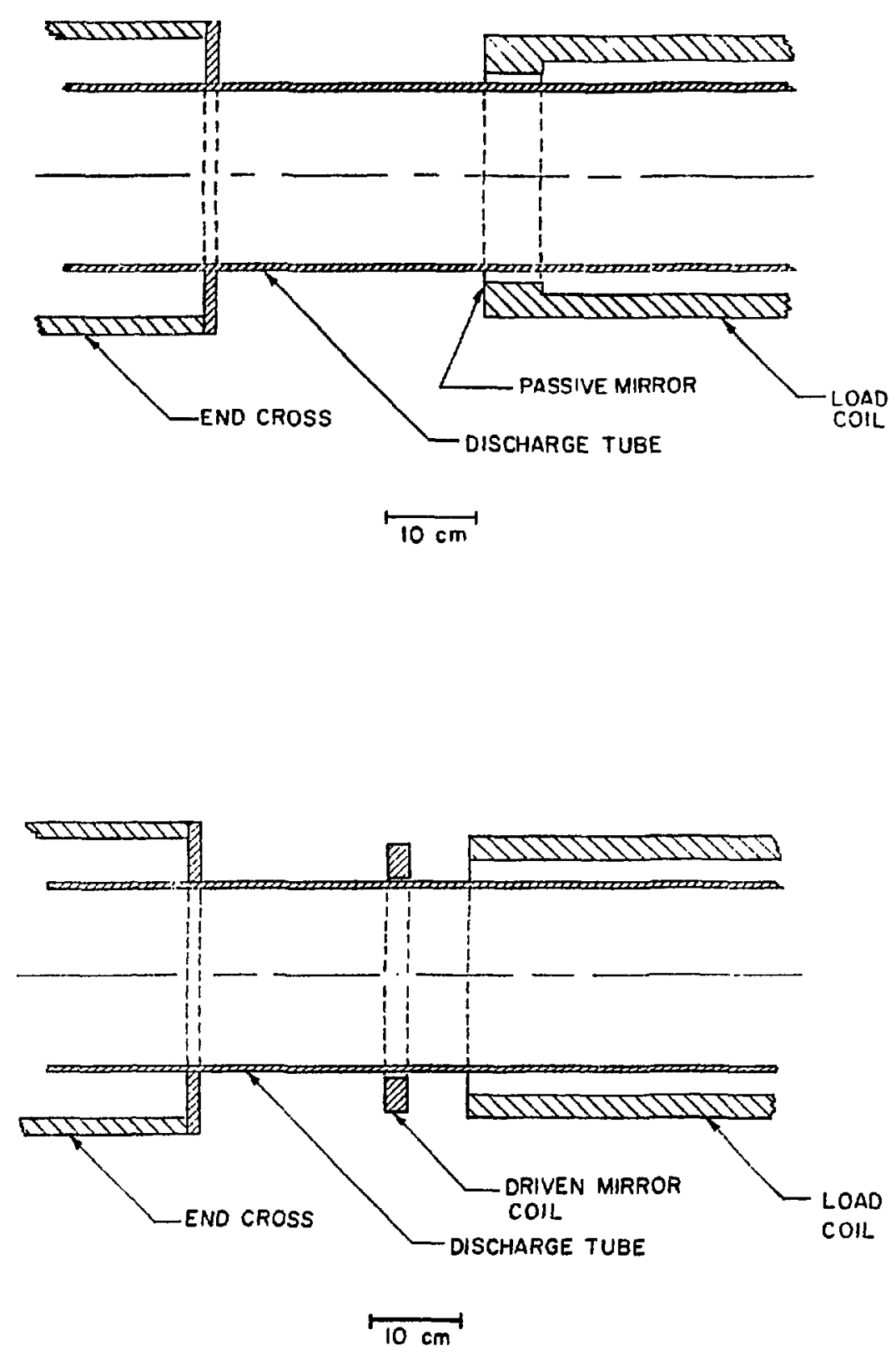

Fig. 1. Schematic diagram showing the (a) passive-mirror coll and (b) the driven mirror coil.

the driven-mirror system, $30 \mu \mathrm{s}$ after implosion, both with and without the driven mirrors energized. 


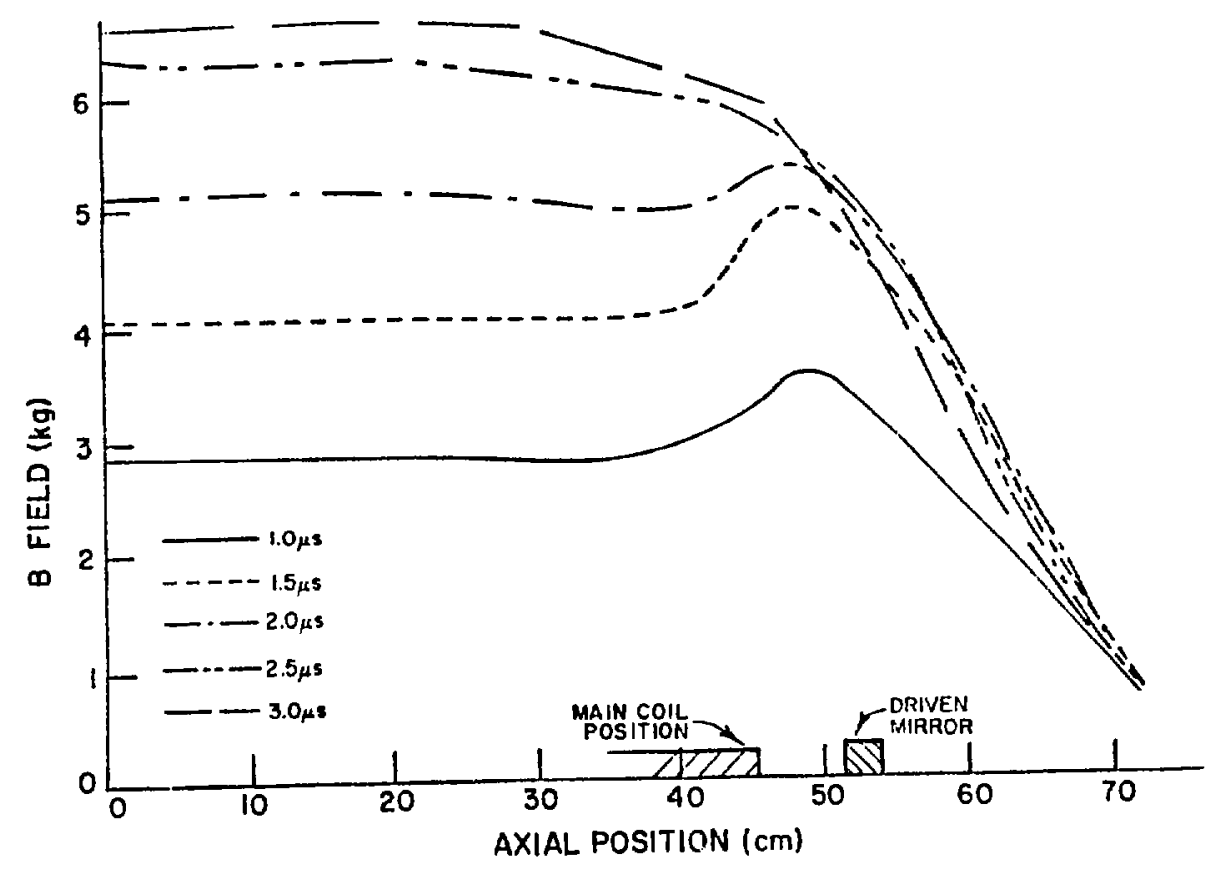

F1g. 2. The measured axial profiles of the magnetic field with driven mirrors for several times during the initial rise of the main field.

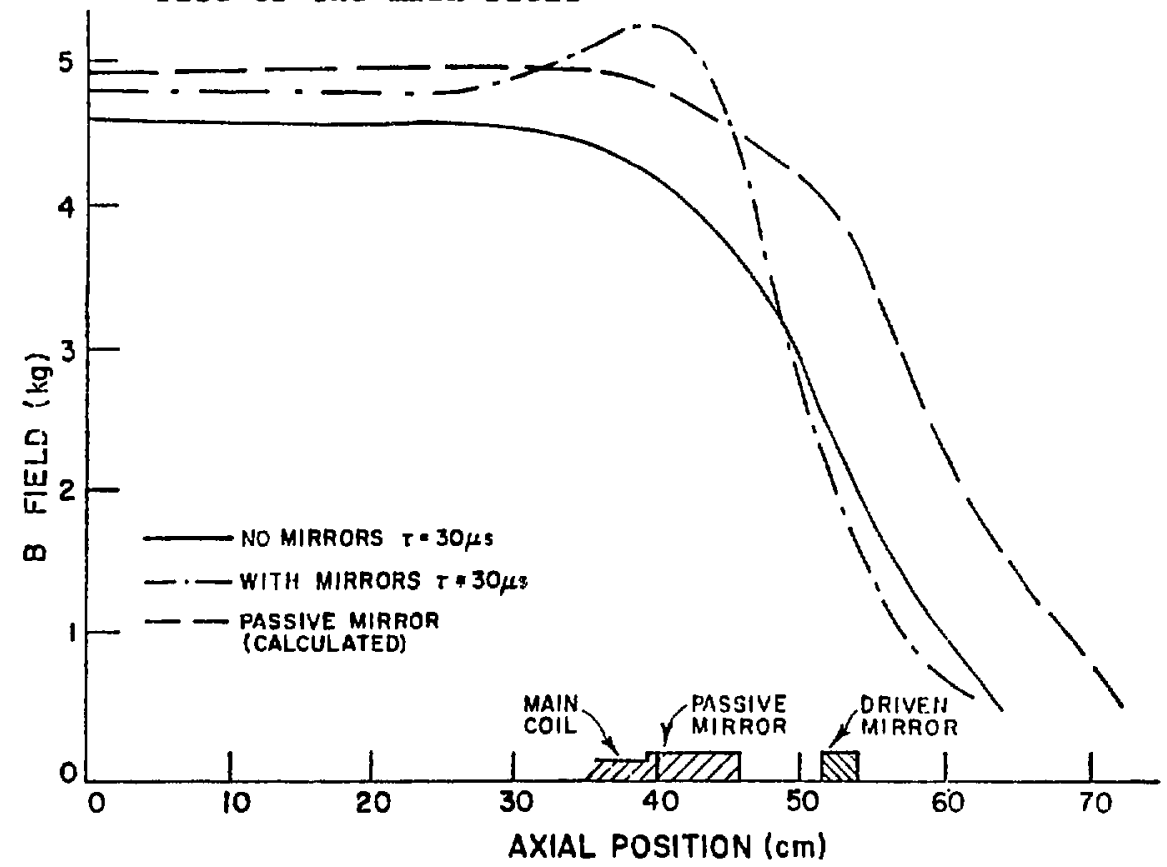

Fig. 3. A comparison of the axial profiles of the field, numerically calculated for the passive-mirror case and measured at $30 \mathrm{\mu s}$ after the implosion for the driven-mirror and no-mirror cases. 
Several observations can be made from these two figures. For optimum plasma lifetimes, no mirror field exists after the peak field is reached. This fact explains the insensitivity of the plasma to the mirror field crowbar. The rise time of the mirror field is only about $2 / 3$ of the main field risetime and substantial mirrors do exist during the Implosion phase. After peak fleld, the curvature of the vacuum fields in the main coil region have the same sign for both driven mirrors and no mirrors (driven mirrors in place, but not energized), but the opposite sign for the passive mirrors. This may partially explain why some parameters, such as optimum filling pressure, are more similar for the driven-mirror and no-mirror cases than for the passive-mirror and driven-mirror cases.

Several diagnostic measurements were made to compare the driven-mirror and nomirror cases with the previously measured passive-mirror case. The major radius, $R$, and minor radius, a, were determined from end-on framing pictures. The obvious inaccuracy of these measurements must be remembered when trying to draw conclusions from the data in Table $I$. The length of the plasma column $\&_{p}$ and the line density Snd through a diameter are obtained with a fractional fringe interferometer. The peak plasma density $n_{m}$ is calculated from the values of $R, a$, and the line density by assuming a rigid rotor profile. The ion temperature $T_{1} 1 s$ determined by the Doppler broadenting of the $C V$ 2271A line measured by a polychromator. The stable time $\tau_{s}$ is defined by the time between the implosion and the onset of the $m=2$ instability (simultaneously observed in the end on framing pictures, $C V$ radiation, and the interferometer). The lifetime $\tau_{L}$ is defined as the time between the Implosion and the annihilation of the reversed field (stmultaneously observed in the end on framing pictures, $C V$ radiation, and the excluded flux feature of the B-field trace). 
TABLE I

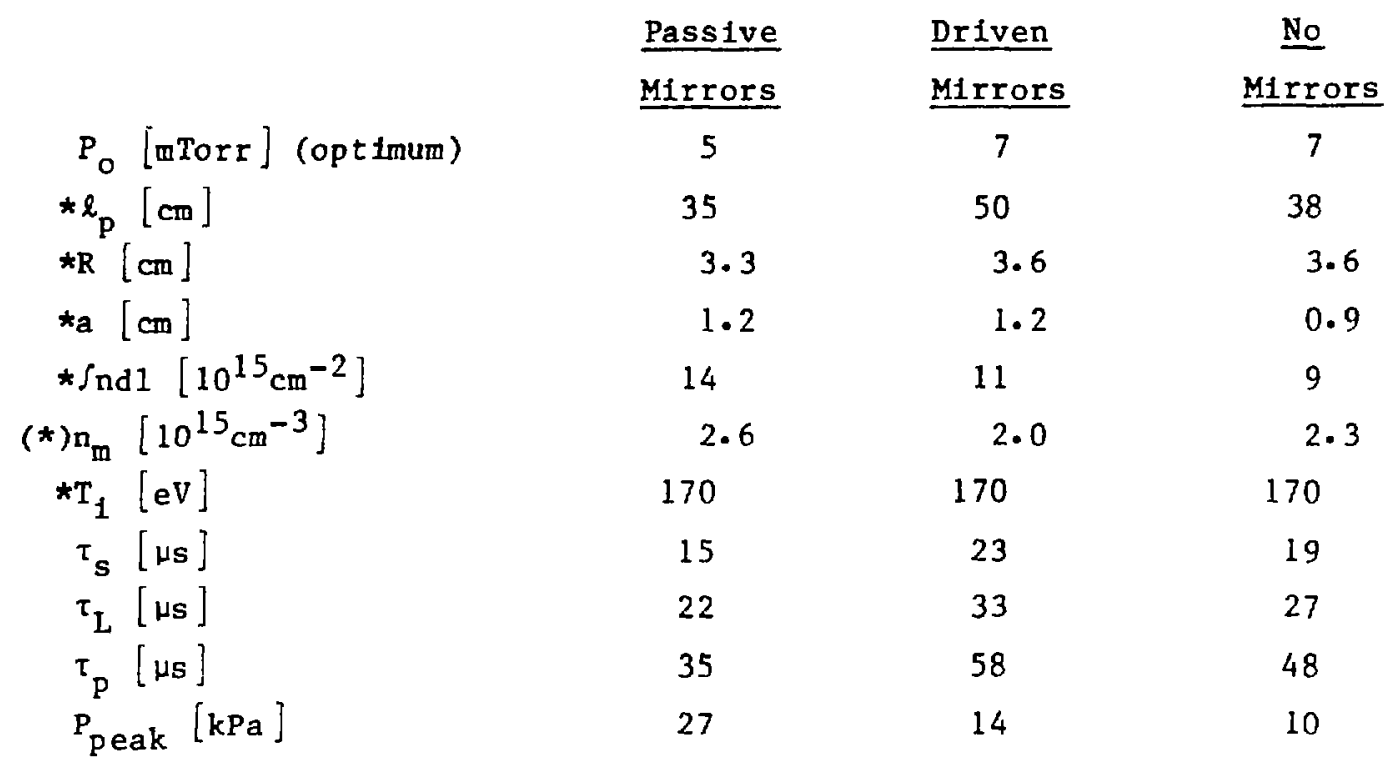

*Measured 10 us after Implosion

In addition to the above diagnostics, pressure probe measurements were made to compare with those taken in the passivemirror case. Radial scans were taken at axial positions that would have the same ratio of local $B-f i e l d$ to the $B-f i e l d$ at the midplane. In particular, the probe was placed at $Z=55 \mathrm{~cm}$ for no $\mathrm{mlrror}, Z=62 \mathrm{~cm}$ for the driven mirrors, and $Z=54 \mathrm{~cm}(8.3 \mathrm{~cm}$ from the main coil) for the passive mirror case, for which the B-field ratio is about $1 / 3$.

Typical on axis pressure signals for the three mirror cases are shown in Fig. 4. Only the passive mirror case exhibits a strong pressure peak early in the plasma lifetime. This behavior is not understood, but might be correlated to the persistance of the mirror fleld after peak field in the passive-mirror case. The maximum pressure, Ppeak, during this early time is recorded in Table $I$. The pressure signal lifetime $\tau_{p}$ is defined as the time between the implosion and the last measured pressure equal to half of $p_{\text {peak }}$.

The pressure profiles in Fig. 5 were obtained for the passive-mirror case. The radial behavior of the driven-mirror case was virtually identical to the $8.3 \mathrm{~cm}$ position in $F 1 g$. 5. The no-mirror case had a much broader radial proflle, apparently arising from an m=l 
PASSIVE MIRRORS

a

DRIVEN MIRRORS

3

NO

MIRRORS

c
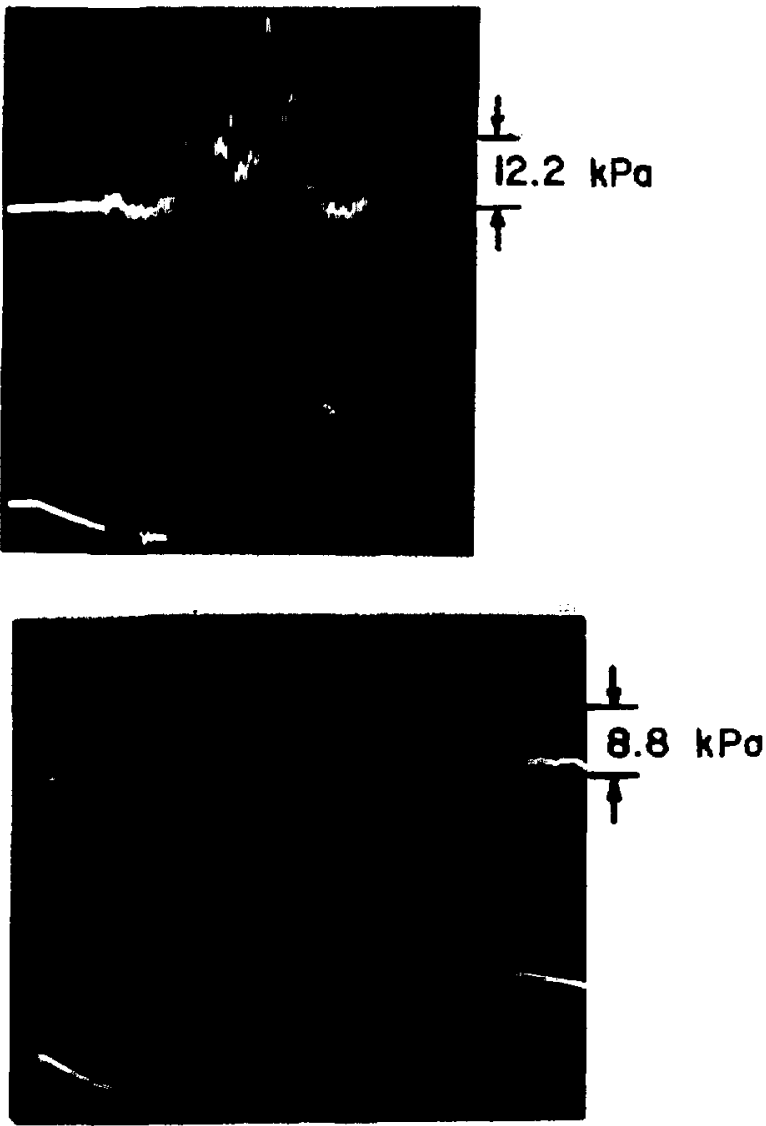

B - FIELD
PRESSURE

B-FIELD
PRESSURE

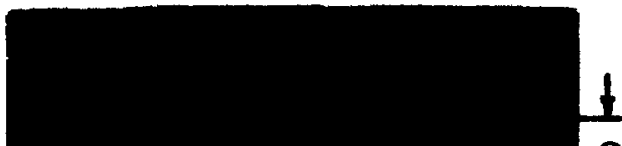

8.8 kPa PRESSURE

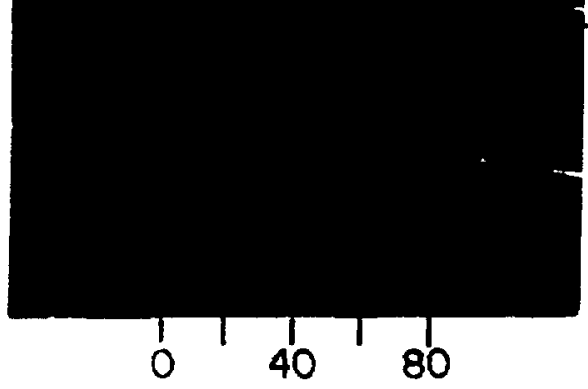

time $(\mu s)$

Fig. 4, Comparison of the on-axis pressure probe signal (upper trace) and the external Bafield (lower trace) for (a) passive mirrors, (b) driven mirrors, and (c) no mirrors. 


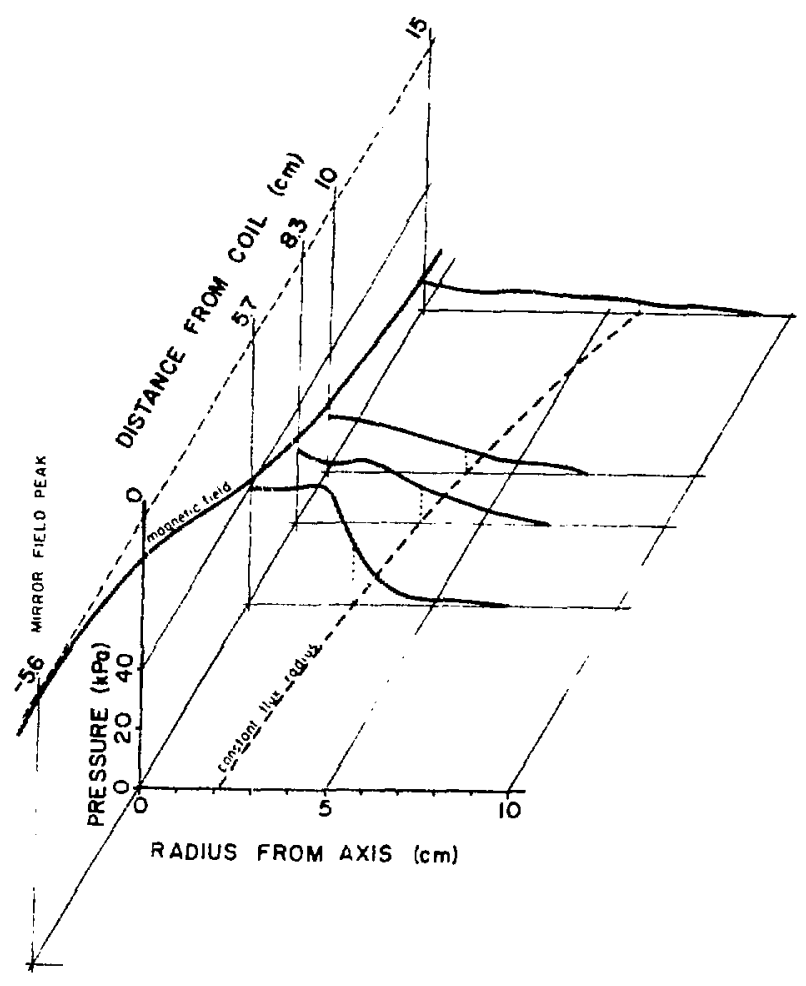

F1g. 5. Spatial distribution of the axial plasma pressure at about 6 us after the implosion for the passive-mirror case.

wobble of substantial amplicude as observed in the end on framing pictures.

It is of interest that the ratios of the characteristic times $\tau_{s}$, $\tau_{L}$, and $\tau_{p}$ are independent of mirrors. In addition, the values of $n_{m}$ and $T_{1}$ and the associated plasma pressure are also essentially Independent of the mirrors. This maximum confined-plasma pressure is slightly less than the pressure assoclated with the measured $5.5 \mathrm{kG}$ flelds, if $\mathrm{T}_{\mathrm{e}} \pm 100 \mathrm{eV}$ is assumed. If the average monentum of the lons hitting the pressure probe is assumed to be proportional to the Ion thermal velocity and independent of tfme, then the integral of the pressure over the cross sectional area of the tube and over time is proportional to the total number of particles in the initial plasma. With this assumption, a rough calculation can show that the pressure probe signal Indicates 2 to 3 times more particles than the density and 
volume measurements indicate, Independent of the mirrors. Even though this pressure probe inventory is consistant with the initial fill, nefther the volume measurements nor the probe measurements are accurate enough to draw the apparently obvious conclusion that $1 / 2$ to $2 / 3$ of the intial fill is being lost during the formation. These conclusions must await the electron temperature and density profile measurement from the Thomson scattering system now being assembled on FRX-B.

In summary, the volume, density, temperature, and pressure probe measurements appear to scale in a self consistant way. However, the relatively poor accuracy does not allow absolute inventory or pressure balance measurements. Contrary to the results of Eberhagen and Grossmanl, measurable differences in the plasmas can be made between the passive-mirror and no-mirror cases ( $P_{0}, \tau_{S}, \tau_{L}, \tau_{P}, P_{p e a k}$ and wobble). In addition, driven mirrors substantially increase the plasma length and lifetime while leaving most other parameters comparitively constant. In particular, increased temperature from axial shock heating, is not observed within the \pm 15 percent experimental error. This result is in contrast to observations by Kurtmullaev ${ }^{2}$, in which independent mirrors of a different design led to enhanced axial shock heating and higher temperatures. Finally, the connection between driven mirrors and increased plasma length and life time is not understood theoretically. A better understanding of the plasma confinement will be necessary before these observed effects can be understood. This increased understanding of plasma transport will require experimental measurements with improved diagnostics over a broad range of operating conditions which can then be matched to theoretical models.

\section{PLASMA TRANSLATION WITH SINGLE DRIVEN MIRROR}

When a single mirror was energized, the plasma formed and contracted axially in about $3 \mu \mathrm{s}$ as in the symmetrical case, but an axtal drift on the order of $3.3 \mathrm{~cm} /$ us caused the plasma to leave the system about 15 us after formation. As expected, the dire-tion of drift can be changed by energizing the opposite mirror coll. The drift velocity increases as the plasma reaches the end of the main coil, apparently because of the strongly diverging fields. The maximum 
a

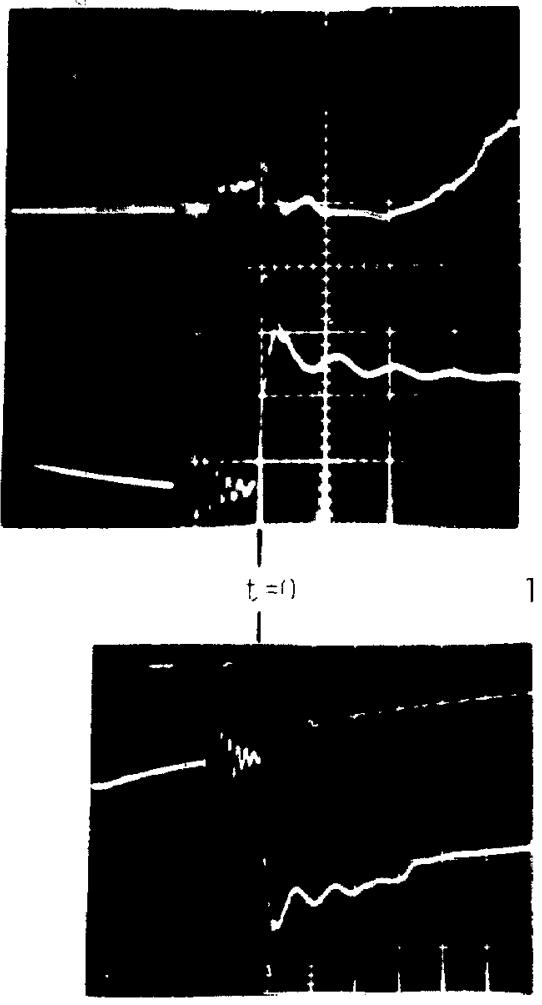

b

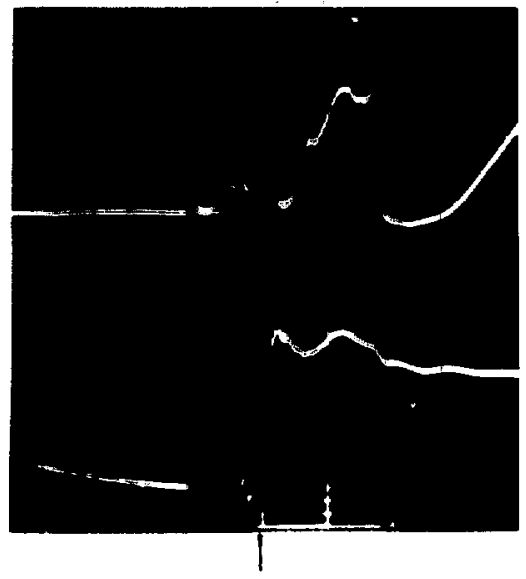

10US/DIV. TIME SCALE

DRIVEN MIRROR

CURRENT

B-FIELD (NEG)

$Z=0 \mathrm{~cm}$

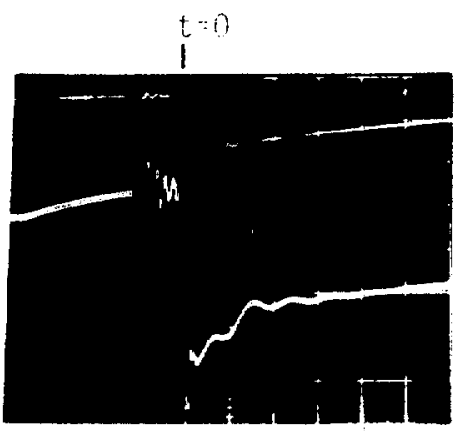

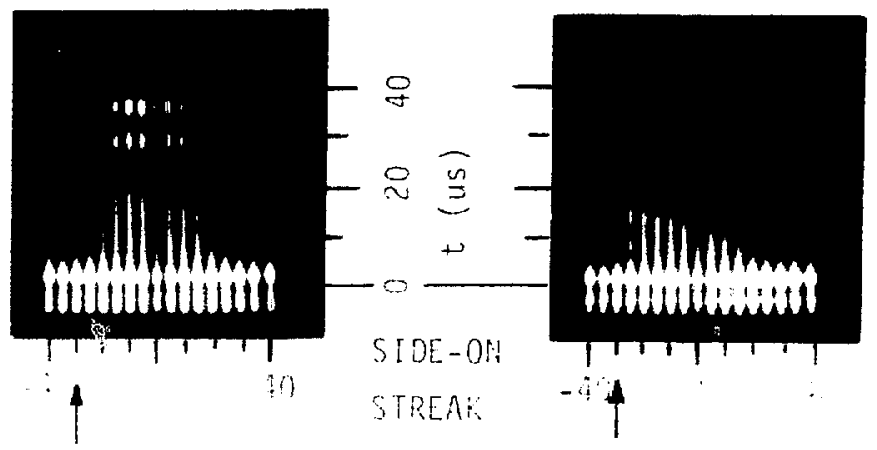

Fig, 6. Comparison of data for a (a) stationary plasma, and a (b) translated plasma, 
observed drift speed is $\sim 7 \mathrm{~cm} / \mu \mathrm{s}$ which is approximately half of the Alfven speed.

The fractional fringe interferometer, external field probes, and the side on framing camera show that a well defined plasma column shrinks to a length of 40 to $50 \mathrm{~cm}$ before 1 t drifts out of the system (see Fig. 6). These measurements indicate that a field reversed plasma has been formed and translated intact. This result is important to the translation and trapping experiment planned for FRX-B. 


\section{REFERENCES}

1. A. Eberhagen, W. Grossman, Z. Phys. 248, 130 (1971).

2. A. G. Es'kov, et al., "Plasma Confinement in a Pulsed System with a Compact Toroidal Configuration", In Proc. Seventh European Conf. Controlled Fusion and Plasma Physics, Lausanne, 1975 (CRPP, 1975) Vol. I, P. 55 . 
(Submitted to the Ninth European Conference on Controlled Fusion and Plasma Physics, Culham Laboratory, Abingdon, England, 1979)

\section{FIET REVERSAL EXPERIMENTS*}

R. K. Linford, W. T. Armstrong, J. Lipson, D. A. Platts, E. G. Sherwood

Los Alamos Scientific Laboratory

Los Alamos, New Mexico, USA

The equilibrium, stability and confinement properties of the reversed-field configuration are being studied in two theta-pinch facilities, FRX-A and FRX-B. The configuration is a torotdal plasma confined in a purely poloidal field geometry. The FRX systems produce highly elongated torif with a major radius $\mathrm{R}=2-6 \mathrm{~cm}$, minor radius a $2 \mathrm{~cm}$, and a total length $\ell \sim 35 \mathrm{~cm}$. Plasma conditions range from $\mathrm{T}_{\mathrm{e}} \sim$ $150 \mathrm{eV}, \mathrm{T}_{i} \sim 800 \mathrm{eV}$ and $\mathrm{n}_{\max } \sim 1 \times 10^{15} \mathrm{~cm}-\mathrm{C}^{-3}$ to $\mathrm{T}_{\mathrm{e}} \sim 50 \mathrm{eV}, \mathrm{T}_{i} \sim 50 \mathrm{eV}$ and $n_{\text {max }} \sim 4 \times 10^{15^{\max }-3}$. The plasma conffguration remains in a stable equilibrium for up to 50 us followed by the destructive $m=2$, rotational instability.

The rotation instability has a delayed onset and slower growth rate with increased density, and correspondingly decreased temperature, scaling on FRX-B. The longest stable period is $\geq 50$ us for 17 mtorr operation. Agreement of experimental and theoretical instability mode frequencies is observed for a variety of theoretical models ( $\omega_{r} \sim 2 \Omega^{*}$ : Freidberg, Pearlsteln, Seyler) over a large range of plasma parameters. However, the rotation source and acceleration rate are not well understood. Scaling of the instability with $n, B, T$ and $a / p_{1}$ has been studied in hopes of identifying the responsible rotation mechanism.

Neither large scale MHD, nor tearing instabilities are observed. Theoretical studies of high beta, shearless interchange modes indicate stability for large elongation and small aspect ratio (Seyler and Barnes). Experimental geometries lie in the calculated stable region, supporting the conjecture that configuration geometry is important to MHD $s=a: 1:$ ty. Experimental stable periods are $\sim 100$ times greater than characteristic MHD e-foldinj times. Furthermore, increasing $a / \rho_{1}$ to $\sim 10$ (corresponding to the 17 mtorr fill. pressure operation on FRX-B) still resulted in no observed MHD instabillties. This is in contrast to past conjecrures that an upper bound of $a / p_{1} \sim 3$ to $5 \mathrm{might}$ exist for MHD stable configurations.

*Work performed under the auspices of U. S. Department of Energy. 
W. T. Armstrong, R. K. Linford. J. Lipson, D. A. Platts, and E. G. Sherwood

\section{Introduction}

The equilitrium, stability, and confinement properties of the reversed field configuration are being studied in two theta pinch facilities referred to as FRX-A, and FRX-B. The configuration is a toroidal plasma confined in a purely poloidal fleld contiguration containing both closed and open field IInes. The FRX system produces highly elongated tori with major radius $R=3-5 \mathrm{~cm}$, minor radius a $\sim 2$ $\mathrm{cm}$, and a full length $2 \sim 35 \mathrm{~cm}$. Plasma conditions have ranged from $I_{e}$ $\sim 150 \mathrm{eV}, \mathrm{T}_{i} \sim 800 \mathrm{eV}$, and $\mathrm{N}_{\max } \sim 10^{15} / \mathrm{cm}^{3}$ to $\mathrm{T}_{\mathrm{e}} \sim 50 \mathrm{eV}, \mathrm{T}_{i} \sim 50 \mathrm{eV}$, and $N_{\max } \sim 4 \times 10^{15} / \mathrm{cm}^{3}$. The plasma remains in a stable equilibrium for up to 50 us followed by an $n=2$ rotational instability which results in termination of the reversed field configuration (RFC). The plasma behavior with respect to equilfbrium, stability, and rotation is consistent with recent theoretical work in these areas.

II. Recent Experimental Results

Before proceeding with a detailed review of experimental data and their consistency with theory, we shall. first briefly describe our most recent experimental results. Independently driven, crowbarred mirror coils were recently included on FRX-A. With optimum mirror timing, it was found that the particle inventory increased as compared with plasmas created with passive mirrors or without any mirrors, the increase being largely due to greater plasma length. It is thought chat the mirrors produce a more localized tearing and reconnection of field lines during the formation phase by providing more favorable fiel.d curvature at the ends. This should contribute to increased inventory. After the first iew microseconds, the mirrors act more like an extension of the implosion coil which would also increase plasma length. An increase in plasma lifetime from 20 to about $35 \mu \mathrm{s}$ is also observed but has not yet been fully explained.

An experiment in plasma translation was performed by disconnecting one of the driven mirrors. Where the remaining mirror was activated $0-5$ us before the implosion, the plasma was observed to translate towards the opposice eno witn an iutiai arifz velocizy of $3.2 \mathrm{~cm} / \mathrm{ks}$, exiting the coil in 15 us. This time is intermediate between the plasma stable time $\left(c_{S}\right)$ and an Alfven transit time $\left(T_{A}\right)$ so that the plasma can undergo a collective translation prior to instability. A 6328 A fractional Eringe interferometer, external fleld probes, and a side on streak camera all indicated a well defined plasma column forming in the center of the implosion coil and then drifting uniformly out of the system.

*Work performed under the auspices of the U. S. Department of Energy. 
End-on framing photography indicated retention of an annular equilibrium as the plasma moves axially.

Thonson scattering has recently been added to FRX-3. The system has all its components rigidly mounted to a single table surface such that all relative alignments remain fixed. To shift the -experiment either radially or axially, the table is translated by means of air bearings. This versatility is permitted because a thre grating polychromator has been employed. This instrument has sufficient rejection at $6943 \AA$ to obviate the necessity of a beam or viewing dump.

Observations were made on the axtal midplane at a constant filling pressure of 17 mtorr. Over the stable lifetime of the plasma, the electron temperature was essentially constant in both time and space at about $100 \mathrm{eV}$ for radif interior to the seperatrix. Relative density was also fairly constant as a function of time at these radii. The ion temperature on the axial midplane was determined by Doppler broadening of the 2271 A line of $\mathrm{CV}$ and after the first 10 us was also $100 \mathrm{eV}$ as required by fairly rapid equipartition. These data indicate that the energy density at any given radial position on the axial midplane is nearly constant in time. This implies that the equilibrium adjusts axially to compensate for losses from this region. The data also reconciles pressure balance quantitatively (to within 20\%) where previous interferometric data at 17 mtorr has been used. The determination of an energy confinement time must await detailed axial scans.

\section{Equilibrium}

Great prograss has been made in modeling the FRX equiliorium both analytically and by means of computer codes.

A $2 D$ sharp bounday calculation by Linford ${ }^{2}$ has yielded results similar to earlier calculations by Wright et $a 1^{3}$, and by Kadish ${ }^{4}$. The model indicates how the equilibrium varies under adiatatic compression, either by the addition of vertical flux, or by a perfectly conducting moving liner. The plasma length $l$ is found to be proportional to $r_{s} 14 / 5$ ( $r_{s}$ is the seperatrix radius) for the first case, and $r_{s} 2 / 5$ for the latter case. These fladings are in excellent agreement for moderate compressions with a $2 \mathrm{D}$ time dependent ideal M.H.D. code developed by Grossmann ${ }^{5}$. The results diverge for large compressions because the equilibrium looses information about the walls ant stinges from an elongated to a roughily circular configuration as described by Kadish, and Stevens ${ }^{6}$.

IV. M.H.D. Stabillty Review

The situation concerning M.H.D. stability has been greatiy clarified. No pure M.H.D. modes are observed during the stable Iizerime even though $z_{s}>1 C 0 s_{A}$ the latter being the minimum time scale for M.H.D. growth. Further, it has been observed that the stable time iacreases with increasing a/p ( $\rho$ is the ion gyro-radius) for our experimental range $3<a / p<10$. This is preciseiy the opposite scaling if finite Larmor radius (FLR) effects were contributing to the stability significantly. 
The 3D M.H.D. simulation work of Anderson ${ }^{7}$ et al, and the analytical work of Batnes and Seyler ${ }^{8}$ has been largely consistent with experimental observations. The $n=0$ mode $(n=$ toroidal mode number) is found to be stable for reasonable pressure profiles in the fixed boundary model but is wall stabilized in the fres boundary model. The $n=1$ sideways mode was found to be stable even without a wall except where very strong mirror fields are included. The $n=1$ tilting mode is never observed in the $3 \mathrm{D}$ computer modeling.

For $n=2,3 D$ computer simulations show stability except for pressure profiles characteristic of over-compressed plasmas. Here the $\mathrm{n}=2$ mode was found to have a relatively small growth race $\gamma^{-1} \sim 30 \mathrm{~T}_{\mathrm{A}}$. For $n>2$ but still not large, the simulations indicate stability.

Local modes have also been considered. For $n \rightarrow \infty$ the $m=0$ mode (m is the poloidal mode number) was found to be stable for closed field line configurations (no shear), and for proper pressure protiles and boundary conditions. Indications are that finfte pressure on the seperatrix is also required. The $m=1$ axial and radial kink modes have been examined by Pearlstein and Newcomb for a Hiil's vortex equilibrium. The growth rates both scale as a/2 so for highly elongated RFC's the growth is small. FLR effect should also help stabilize these modes. For $\mathrm{n} 22$ all modes were stable. In all cases, elongation of the equilibrium was favorable for stability.

\section{Rotation}

The plasma lifetime is limited by the onset of an $\mathbf{n}=2$ rotational instability which has been treated theoretically by seyler 10 using a Vlasov fluid code, and by Freidberg and Pearlsteinll using an FLR expansion. In Seyler's rodel $-\omega_{2} / 2 t=1.64$ where $\omega_{r}$ is the real part of the perturbation frequency, and $\Omega^{*}$ is the diamagnetic drift frequency. For FRX, this ratio is about 2.0 where $\omega_{r}$ is determined by end on framing photography. The vlasov fluid model also indicates a critical value of $\alpha \equiv-\Omega / \Omega^{*}=1.55$ for stability where $\Omega$ is the ion rotational frequency. This is not in agreement with the experimentally determined value of 0.4 , however $\Omega$ is determined experimentally by measuring the doppier shift of the $2271 \AA$ line of $\mathrm{CV}$. It is thought that the 1mpurity profile is much more diffuse than the deuterium ion profile and that the CV measurement is not reflective of the deurerilm rotational velocity. This is supported by the theoretical criterion that $\omega_{r} \leqslant n \Omega$ an inequality not satisfled for the experimental data. The critical $\Omega$ however has been observed to scale with $\Omega *$ as predicted.

The most plausible explanation for the spin-up of the plasma has also been advanced by Seyler ${ }^{12}$ where the preferential loss of particles with negative angular momentum is considered. These particles are found to be located either outside the seperatrix or encircling the axis, precisely the locatjons of anticipated large losses.

VI. Transport/Scaling

Transport has been the least satisfactorily studied araa both experimentally, and theoretically. The energy confinement time during the stable period is of the order of tens of microseconds shich is 
significantly shorter than a classical diffusion t1me. One candidate for enhanced eransport is like particle (ion-ion) diffusion ${ }^{13}$ which can proceed rapidly when the density gradient length is of the order of an ion gyro-radius. Also posstble is anomalous transport due to the lower hybrid drift instability ${ }^{12}$ which is driven by strong pressure gradients. A ID hybrid code is being developed by Hamasaki 14 to consider both classical and anomalous transport but has not yet ylelded defintive results.

Experimentally, the study of transport and scaling is limited by the relatively restricted parameter range of existing machines. A larger experiment called FRX-C has been proposed to alleviace the difficulcy.

\section{Conclusions}

Considerable progress has been made in defining the equilibrium, stability, and rotational properties of the RFC. These efforts have been aided by the addition of Thonson scattering to FRX-B, and by substantial new results from the theoretical community. Much remains to be done both experimentally and theoretically in understanding transport and $s c 311 \mathrm{gg}$ with respect to micro-turbulence, resistive instabilities, M.H.D. stability, and equilibrium.

\section{References}

1. R. Siemon, Appl. Optics 13, 697 (1974)

2. R. K. Linford to J. Lipson, private communication

3. J. K. Wright, R. D. Medford, and B. Chambers, Plasma Physics 12 , 242 (1951)

4. A. Kadish, Conf. Record of 1979 IEEE Intl. Conf. on Plasma Science, p. 140

5. N. Grossmann, W. Hameiri, Proc. of the Sherwood yeeting on Theoretical Aspects of CTR Research, paper 2B9 (1979)

6. A. Kadish, D. C. Stevens, Nuclear Fusion 14, 821 (1974)

7. D. V. Anderson, W. A. Newcomb, D. C. Barnes, C. E. Jeyler, Pracof the Sherwood Meeting on Theoretical Aspects of CTR Research, paper $2 \mathrm{~A} 4$ (1979)

8. D. C. Barnes, C. E. Seyler, LASL Rpt, LA-UR-7?-13 (1978)

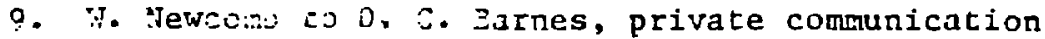

10. C. E. Seyler, LASL Rpt. EAl-ITR-7o-185 (1979)

11. J. P. Freidberg, L. D. Pearlstein, Phys. Fluids 21, 1207> (1978)

12. C. E. Seyler to R. K. Linford, private comunication

13. 11. Tuszewski, A. J. Lichtenberg, Phys. Fluids 20, 1263 (1977)

14. S. Hamasaki, Conf. Record of 1979 IEEE, IntI. Conf. on Plasma Science, p. 143 